\title{
FLORIDA STATE BOARD OF CONSERVATION
}

J. T. HURST, Supervisor

FLORIDA GEOLOGICAL SURVEY

HERMAN GUNTER, Director

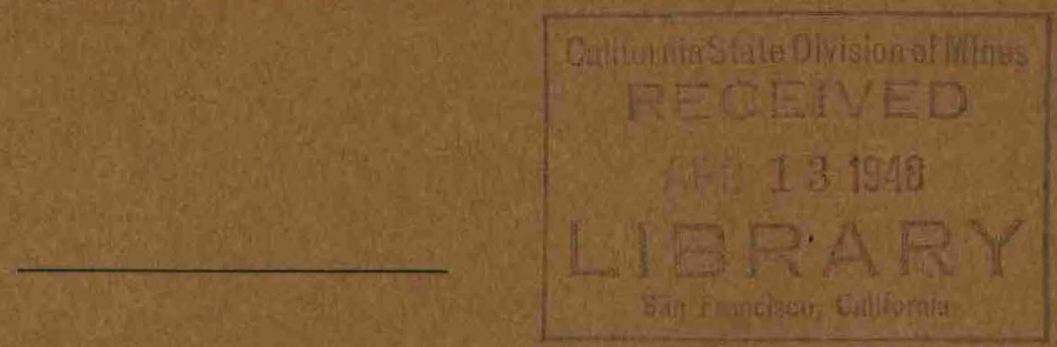

INFORMATION OIROULAR-NO. 1

\section{GXPLORATION FOR OIL AND GAS IN FLORIDA}

By

HERMAN GUNTER, Director

Florida Geological Survey

TALLAHASSEE, FLORIDA

JANUARY 1, 1948 



\title{
FLORIDA STATE BOARD OF CONSERVATION \\ J. T. Hurst, Supervisor
}

FLORIDA GEOLOGICAL SURVEY

Herman Gunter, Director

INFORMATION CIRCULAR - NO. I

EX PLORA TION FOR O I L AN D GAOA IN FLORI DA

\author{
By \\ Herman Gunter, Directer \\ Florida Geological Survey
}
Tallahassee, Florida
January 1,1948

liimeographing actuaily

completed February 14, 1943. 


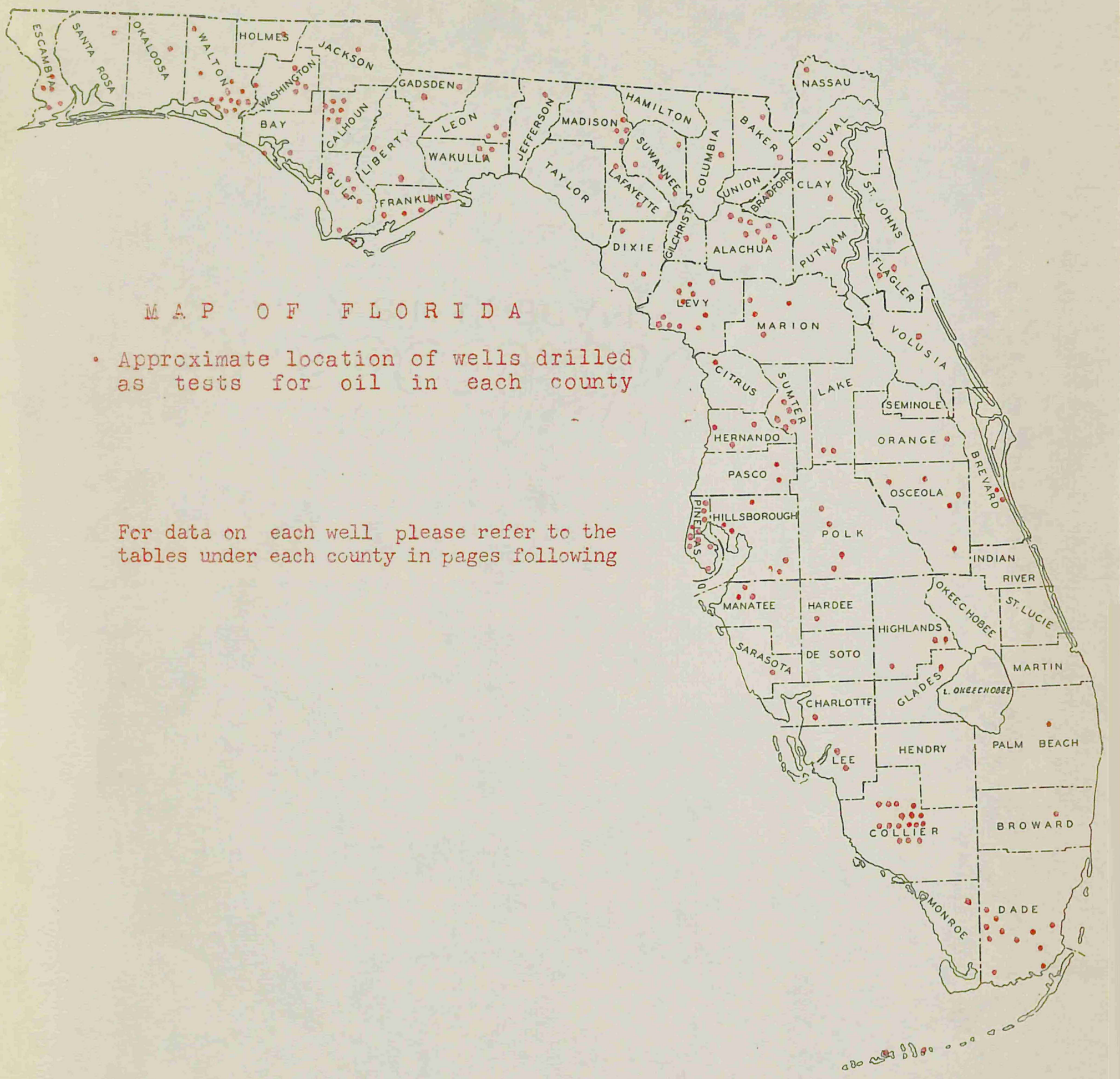


Introduction. ....................................... History of Development............................... Regulatory Measures................................... Summary........................................... Explanation of tables.................................. Acknowledgements.................................... Alachua County....................................... Baker County........................................... Bay County............................................ Bradford County........................................ Brevard County...................................... Broward County..................................... Calhoun County....................................... Charlotte County......................................... Citrus County......................................... Clay County............................................ Collier County....................................... Columbia County....................................... Dade County.......................................... Dixie County......................................... Duval County......................................... Escambia County...................................... Flagler County..................................... Franklin County.................................... Gadsden County........................................ Gilchrist County...................................... Glades County....................................... Gulf County......................................... Hardee County.......................................... Hernando County...................................... Highlands County.................................... Hillsborough County................................ Holmes County.......................................... Jackson County...................................... Jefferson County....................................... Lafayette County...................................... Lake County........................................ Lee County............................................ Leon County.......................................... Levy County........................................ Liberty County......................................... Madison County....................................... Manatee County......................................... Marion County........................................... Monroe County......................................... Nassau County......................................... Okaloosa County....................................... Orange County....................................... 
Osceola County......................................

Palm Beach County........................................

Pasco County.............................................

Pinellas County.......................................

Polk County..........................................

Putnam County.........................................

Santa Rosa County .......................................

Sarasota County.........................................

Sumter County...........................................

Suwannee County.........................................

Volusia County......................................

Wakulla Countjr.....................................

Walton County......................................

Washington County.................................

\section{IIIUSTRATIONS}

Figures

1. Map of Florida, approximate location of wells drilled as tests for oil in each county...............

2. Location of wells in Sunniland Oil Field and in nearby prospects..............................

Tables

1. Completion data on the producing wells in the Sunniland Field...............................

2. Production of oil from wells in Sunniland Field from September 1943 to December 31, 1947 ......... following 


\section{EXPLORATIONS FOR OIL AND GAS IN FLORIDA \\ Herman Gunter, Director \\ Florida Geological Survey \\ Tallahassee}

\section{INTRODUCTION}

It seems that there always has been an interest in the possibility of producing oil and gas in Florida, if such interest is measured by the number of requests for information received by the Florida Geological Survey. Through the years the Survey has published papers and reports dealing with the petroleum possibilities, structure and the geology of the State, but recently there has been a growing demand for more complete information about the early explorations. It is hoped this mineographed report will supply most of the desired data, and make a ready reference for the information which is available in Survey files. In this connection it may be said that we have endeavored to obtain dependable data on the wells drilled years ago, but we invite corrections and additional information from any who may be in a position to furnish such. Especially welcome would be driller's logs, newspaper clippings or notes on wells drilled prior to 1910. Such records would supplement the meager and unverified data that we now have on these earliest operations.

Prior to June 5, 1945, Florida had no law regulating the drilling for oil or gas. Since the establishment of the Florida Geological Survey in 1907, however, attempts have been made to record details of all exploratory drilling and also to gather data on explorations made prior to 1907. Because there was no law regulating drilling, information was obtained only through the interest and willingness of the operat.jrs to give essential data while the well was in progress, or to submit it after completion. With but few exceptions, cooperation in this has been most satisfactory and agreeably 
rendered. When a request has been made by the operator or owner to hold data confidential for a stated period, this has been done in every case.

It can be recorded with much satisfaction that the drillers of water wells have been cooperative and willing to submit inf smation and samples of the drill cuttings from wells drilled in almost every portion of Florida. Through the years our persistent efforts to procure such data have resulted in a file of samples and cores from more than 1650 water wells and oil test borings. These data have proved of inestimable value during recent years in the accelerated search for geological and structural information bearing on the origin and accumulation of oil. These excellent records were at least a factor contributing to the decision of several major oil companies to locate their offices and district headquarters in Tallahassee.

\section{HISTOIYY OF DEVELOPMENT}

As stated, the recording of data for the wells here listed began with the organization of the Survey in 1907. However, in those earlier years records were not as complete as they now are, and there was less inclination on the part of the operators to release their records. Then, too, the Survey organization was more limited in personnel and did not visit the operations. Consequently, the records of those early drillings leave much to be desired. After wells are abandoned it is difficult, if not impossible, to get reliable information. This is certainly true in F'lorida, especially for those earlier wells, the records of which have become so distorted with age as to make them of questionable vaiue. In all instances efforts have been made to verify and re-check the names of the sperators, the lucation, the depth, and other data by reference to old newspapers, by visits to persons who may have kept records of such drillings, by interviewing drillers, surveyors, and by correspondence. Sometimes the sum total of such efforts have been satisfactory, yet for certain wells verified data is still lacking. 
As nearly as has been established, the earliest test for oil in Florida was about 1900 or 1901, at Pensacola, Escambia County. This well was drilled by the Escambia Oil Company at the foot of Palafox Street to a depth of 1,320 feet. Apparently this rig was then sold to another local company, Pensacola Development Company, and two wells were drilled: No. 1 about 5 miles west of Pensacola in what is now New Warrington, and No. 2 on East Hill in Pensacola at the intersection of Tenth Avenue and Mallory Street. These wells attained depths of 1702 and 1620 feet, respectively. Driller's logs on both of these have been preserved and published in Water Supply Paper 102 of the U. S. Geological Survey, 1904, and in the Fourteenth and Seventeenth Annual Reports of the Florida Geological Survey.

Upon completion of these vells, interest in this west Florida area seemed to diminish temporarily and the next wells of record were drilled in Sumter County, central peninsular Florida, in 1903. Two wells were put down, the first apparently being abandoned at about 1200 feet because of drilling difficulties, and the second completed at a depth of 2002 feet. It is not known why these particular areas in western and central peninsular Florida were selected, unless it was on the basis of general surface geology. Sumter County lies within the region referred to in literature as the "Ocala Uplift", being that part of Florida where the upper Eocene limestones are exposed at the surface, or only thinly mantled by more recent sediments. It has long been recognized as a regionally geologic high area, and could therefore have attracted the attention of the early explorers for oil in the same manner as it has intrigued those in more recent years.

From these early beginnings the interest in the possibility of oil or gas in Florida has waxed and waned through the years. From rather close familiarity with the oil prospecting developments in Florida for many years 
it is evident that some of this interest was prompted by enthusiastic, overzealous, professional promoters whose first concern was personal gain. By far the larger number, however, made serious attempts to find oil, as is evidenced by the several deep wells drilled by operators of highest integrity whose motives were never questioned. Even the first wells of record in Escambia County were of this character and for that early day the depths of these were quite impressive, ranging from 1320 feet to 1702 feet.

In 1939 a well was completed near Pinecrest, Monroe County, at a depth of 10,006 feet. This was the first well in Florida to attain that depth and, although it did not produce oil, it was drilled under the constant supervision of a trained geologist who kept detailed data on the formations penetrated and gave personal attention to the collection of cuttings and cores, as well as other pertinent data. The complete information yielded by this deep test, terminating as it did in sedimentary formations at more than 10,000 feet, drew the attention of major oil companies to the potential possibilities of Florida. The year 1939 is, therefore, an appropriate dividing point between early prospecting in Florida and the present period of prospecting by major companies. During this first period from about 1900 to and including 1939, there were at least 87 tests drilled. Many of these were very shallow and, except for having been drilled by operators who so designated the well, could hardly be labelled as "oil tests". But there were many wells drilled during this first period that are acknowledged to be as completely tested, adquately sampled, and recorded as any in the State.

As an index, however, of the increased interest and activity in oil prospecting, the period from 1940 to 1948 records the completion of 104 wells, with six wells in process of being drilled on January 1, 1948. It was on September 26, 1943, that the Humble Oil and Refining Company completed their 
Gulf Coast Realties Corporation No. I well at a total depth of 11,626 feet, and brought in the first oil well in Florida. The initial production was 97 barrels of oil with 425 barrels of salt water, the oil having a gravity of 20.8 A.P.I. This well continued its production until May 14, 1946, when on account of decrease in production it was plugged and converted into a salt water disposal well.

This first well did not flow, as was also the case with the second producing well. However, this Sunniland Field on January 1, 1948 had a total of five producing wells - one purping and four flowing. These were as follows:

Well No. 4 - 11,597 feet. Initial production 257 barrels. Pumping Well No. 5 - 11,578 feet. Initial production 518 barrels. Flowing Well No. 6 - 11,578 feet. Initial production 225 barrels. Flowing Well No. 8 - 11,576 feet. Initial production 527 barrels. Flowing Well No.11 - 11,573 feet. Initial production 120 barrels. Flaning

On January 3, 1948, Gulf Coast Realties well No. 10 was completed at a depth of 11,574 feet, with production of 175 barrels of oil daily. This now gives Florida a total of six producing wells in its one field, Sunniland, in Collier County.

The total production of oil in Florida from its discovery, Soptember 26, 1943, through December 31, 1947, was 359,740 barrels.

\section{REGULATORY MEASURES}

An act to regulate and control the drilling and development of sil and gas in Florida was introduced and passed by the Legislature of 1945, and received the approval of the Governor on June 5, 1945. This is Chapter 22819 (No. 305) Laws of Florida 1945. This law was patterned after a model prepared under the supervision of the Interstate Oil Compact Commission with such changes as ware deemed advi.sahl.e after public hoarings were held, con- 
ducted by the Attorney General and the committee from the Florida Bar Association. It has had the careful scrutiny of members of the legal department of at least two of the major oil companies. It is considered as good a conservation act as could be devised at the time. So far, it has proved adequate for development in Florida and appears to meet the satisfaction of all concerned. Copies of this law, together with copies of the rules and regulations that have been adopted by the State Board of Conservation as well as copies of forms 1 to 12 applicable in the rules, can be obtained upon request addressed to Honorable R. A. Gray, Secretary of State and Secretary Oil Division, State Board of Conservation, Tallahassee, or to Dr. Herman Gunter, State Geologist and Assistant Secretary Oil Division, State Board of Conservation, Tallahassee. Such requests will receive prompt attention.

In 1947, the Legislature passed an act levying a tax on oil and gas produced in Florida. This is Chapter 23883 (ivo. 269), Laws of Florida 1947, and copies may be obtained by addressing requests to the Secretary of State Tallahassee, Florida. This act became a law without the Governor's approval and wes filed in the Secretary of State's office June 3, 1947.

\section{SUMMARY}

From the more or less complete information at hand it is known that up to January 1, 1948, approximately 191 wells have been drilled for oil or gas. There were on January 1, 1948, six wells incompleted and drilling, and in the tabulated data there is included some few established locations that may be drilled in the future. Completions have likewise been noted on some of the wells that were drilling on January $i, 1948$, since this report was not completely mimeographed until the latter part of the month.

The wells drilled prior to 1939 were largely on general geologic evidence or on "hunches" such as the topographic and physiographic 
resemblance to certain oil fields elsewhere, or because of the use of some particular "oil finding" machine that in the hands of the operator would predict the occurrence of petroleum. Care should be used in distinguishing these pseudo-scientific methods from the bone fide geophysical exploration methods of recent years. It should be borne in mind that the accepted and credited geophysical methods do not reveal or indicate the presence of oil or gas, but provide another valuable tool in the hands of trained technicians in determining subsurface structural conditions.

From 1940 to the present the exploration for oil and gas has been intensively carried on by the major oil companies. The methods of exploration have included not only surface geology, but also the study of samples from wells throughout the State, detailed field work within defined areas including geophysical exploration and intensive structural drilling. In the drilling of these structural tests the operations are carried to some well known zone often necessitating tests of 1500 to 2000 and more feet deep. While statistics are not available, it is judged that several hundred such tests have been put down by the major oil companies working in different areas. In addition, much geophysical prospecting has been accomplished and some is in progress. Different methods have been tried, some apparently with more satisfactory results than others. The deep drilling already accomplished has yielded information that is, however, more dependably interpretable than the geophysical exploration results. With it all, however, Florida's geological history is gradually being fathomed, although there are many perplexing problems still unsolved.

This highly creditable and excellent work by the various companies and individuals has resulted in a store of information abnut geslogical conditions in Florida that necessitates much study and time to assimilate. Some of the 
exploratory work resulted in conclusions immediately favorable as, for instance, the first well drilled in the Sunniland Field proving production. In other areas no production has been found, but the geologic and stratigraphic data have been very helpful and revealing. That production so far has not been found outside the Sunniland Field apparently has not discouraged the major oil companies, for prospecting continues generally throughout the state. The Sunniland Field may receive more intensive developments so that the possibilities in that southern Florida region can be more completely determined.

Florida is a large state with an areal extent of about 58,000 square miles. The sum total of 194 wells is an unimpressive number for so large an area, and it is therefore no wonder that results have not been more favorable. Thet production will not follow continued exploratory efforts can not be forecast and, from the activity currently going on, it must be concluded that those carrying on such explorations have a basis for them - a wild cat territory with ample thickness of sedimentary formations that could yield oil if favorable traps can be found. Testing in progress may give some of the answers. Problems such as source beds, their position and extent, the condition of permeability and involved lithologic variations, and the usual questions of structure and stratigraphy always present in new regions, all have presented themselves in Florida. The solution to these problems apparently lies in data to be revealed through more drilling.

\section{EXPLANATION OF TABLI'S}

The tabulation which follows is arranged alphabetically according to counties. Unden each county the individual operations have been arranged chronologically as nearly as possible. Data for each well includes: 1) the 
company or owner; 2) farm or well name; 3) location; 4) elevation; 5) year completed; 6) depth; 7) remarks. And also there is included the well number assigned by the Florida Geological Survey. In this there will be found two designations: W-975 and Vgi-386. The first, W-875, means that the Survey has samples and drilling data; the second, Wgi-386, means that we have no samples but may have a driller's log, analysis of water, or other information.

The records on file in the Survey offices are open for ready reference and available to the public. In some instances current data is held for a definite period in the confidentinl file, if the operator makes such request and the State Board of Conservation approves. Should anyone wish to study the open file of cuttings and cores, the Survey will be glad to make them available.

\section{ACKNOWLEDGEMENTS}

We wish to express appreciation and hearty thanks to all drilling contractors, representatives of oil companies, individual operators, and others, who have so willingly and splendidly cooperated in the Survey's effcrt to accumulate these data. Alsc, to those who have painstakingly delved into whatever original record they might have in order to help. us complete fragmentary information and to verify inaccurate or uncertain details, we extend thanks and acknowledge with appreciation their helpful service. We have a very creditable file of records which is continuously adding to our knowledge of the geology, structure and stratigraphy of Florida. 


\section{ALACHUA COUINTY}

\begin{tabular}{|c|c|c|c|c|c|c|c|}
\hline $\begin{array}{l}\text { Fũ̃ } \\
\text { No. }\end{array}$ & COMPANY Or OWNERS & $\begin{array}{l}\text { FARU or } \\
\text { FELL NAWE }\end{array}$ & LOCATION & $\begin{array}{l}\text { ELEV- } \\
\text { ATION }\end{array}$ & $\begin{array}{l}\text { YEAR } \\
\text { CONi- } \\
\text { PLETED } \\
\end{array}$ & DEPTH & REMARKS \\
\hline Wigi- & $\begin{array}{l}\text { Coastal Petroleum } \\
\text { Co.(Arnold, Allen, } \\
\text { Green and kialcolm) }\end{array}$ & . & $\begin{array}{l}\text { Sec. } 27, \mathrm{~T} 9 \mathrm{~s}, \mathrm{R} 2 \mathrm{lE}, \\
\text { orange Heights, } \\
4 \mathrm{mi} \mathrm{sW}-23 \mathrm{ft} . \\
\text { from } \mathrm{Fi}-206\end{array}$ & $!$ & 1927 & 450 & $\begin{array}{l}\text { No samples but a } \\
\text { Driller's log by } \\
\text { Gray Jell and Pump } \\
\text { Co., Jacksonville }\end{array}$ \\
\hline $\begin{array}{l}387 \\
387\end{array}$ & $\begin{array}{l}\text { Coastal Petroleum } \\
\text { Co.(Arnold, Allen, } \\
\text { Green and halcolm } \\
\\
\\
\end{array}$ & Number 2 & $\begin{array}{l}\text { Sec. } 27, \text { T9s, R2lE, } \\
\text { Orange Heights, } \\
4 \mathrm{mi} \text { in }-200 \mathrm{ft} . \\
\text { from Wgi-206 }\end{array}$ & $\vdots$ & 1927 & 662 & $\begin{array}{l}\text { No samples preserved } \\
\text { 'but some were seen at } \\
\text { ithe well Narch } 1,1928 \\
\text { These showed: } 0-124 \\
\text { phosphatic marl - } \\
\text { Hawthorn Niocene; } \\
\text { l24-365' Ocala Eocene; } \\
\text { below } 365^{\prime} \text { brownish } \\
\text { icrystalline limestone } \\
\text { 'Driller's log. }\end{array}$ \\
\hline Whi- & $\begin{array}{l}\text { Arnold, Allen, } \\
\text { Green and lialcolm }\end{array}$ & & $\begin{array}{l}\text { sec. } 27, \text { T9s, R21E, } \\
\text { orange Heights, } \\
4 \mathrm{mi} \text {. } \mathrm{WW}\end{array}$ & ! & 1928 & $676^{+}$ & $\begin{array}{l}\text { No eamples or other } \\
\text { definite data }\end{array}$ \\
\hline & C. L. Arter & $\begin{array}{l}\text { National Tur- } \\
\text { pentine \& } \\
\text { Pulpwood Corp. } \\
\text { No. } 1\end{array}$ & $\begin{array}{l}\text { Sec. } 11, \mathrm{~T} 83, \mathrm{R} 19 \mathrm{E}, \\
\text { Lacrosse, } 3 \mathrm{mi} . \mathrm{S}\end{array}$ & 160 & 1944 & 398 & $\begin{array}{l}\text { Drill atuck, pulled } \\
\text { derrick in. } \\
\text { Abandoned }\end{array}$ \\
\hline$w-875$ & $\begin{array}{l}\text { National Turpen- } \\
\text { tine \& Pulpwood } \\
\text { Corp. } \\
\end{array}$ & & $\begin{array}{l}\text { Sec. } 32, \text { TSS, R2OE, } \\
\text { near Paradioe a- } \\
\text { bout } 6 \text { mi. N of } \\
\text { Gainesville }\end{array}$ & $180^{+}$ & 1944 & 802 & $\begin{array}{l}24 \text { samples from } 10 \mathrm{ft} \\
\text { to } 802 \text { ft. Drilled } \\
\text { for. National Containet } \\
\text { Corp., Jacksonville }\end{array}$ \\
\hline
\end{tabular}


ALACHUA COUNTY - Continued

\begin{tabular}{|c|c|c|c|c|c|c|c|}
\hline FGs & COAPANY Or O $O N E R S$ & $\begin{array}{l}\text { FARM or } \\
\text { WELL NAIME }\end{array}$ & LOCATION & $\begin{array}{l}\text { DLEV- } \\
\text { ATION }\end{array}$ & $\begin{array}{l}\text { YEAR } \\
\text { COM- } \\
\text { PLETED } \\
\end{array}$ & DEPTH & REMARKS \\
\hline 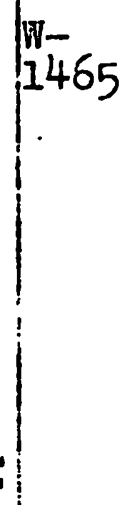 & $\begin{array}{l}\text { Tide Nater Associa- } \\
\text { ted oil Company }\end{array}$ & R. H. Cato No.I & $\begin{array}{l}\text { Sec.23, T85, Rl8E, } \\
\text { Alachua } 1 \mathrm{mi} \text {. } \$\end{array}$ & $\begin{array}{l}102 \mathrm{Grd} \\
112 \mathrm{DF}\end{array}$ & 1947 & $\begin{array}{l}3,150 \\
.\end{array}$ & $\begin{array}{l}10 \text { samples from } 10 \\
\text { to } 75 \text { ft. No samples } \\
\text { from } 75 \text { to } 1568 \text { ft. } \\
171 \text { samples from } \\
1568 \text { to } 3150 \text { ft. } \\
\text { Cores from No. I } 3 \\
1203-1208 \text { to No. } 14 \\
33140-3150 \text {. Schlum- } \\
\text { berger from } 98 \text { to } \\
3146 \text { Driller's log } \\
\text { from o to } 3150 \text {. }\end{array}$ \\
\hline 1472 & $\begin{array}{l}\text { Tide Water Associa- } \\
\text { ted Oil Company }\end{array}$ & $\begin{array}{l}\text { J. A. Phifer } \\
\text { No. I }\end{array}$ & $\begin{array}{l}\text { Sec. } 24, \text { T9s, R2le, } \\
\text { Orange Heights, } \\
2 \mathrm{mi} . \pi\end{array}$ & $\begin{array}{l}\text { 122Grd } \\
132 \mathrm{DF}\end{array}$ & 1947 & 3,228 & $\begin{array}{l}253 \text { samples from } 10 \\
\text { to } 3223 \text { ft. Cores } \\
\text { from No. } 1 \text { 3 } 2900- \\
2910 \text { to No. } 30 \$ 3 \\
3227-3228 \text {. Schlum- } \\
\text { berger from } 197 \text { to } \\
3223.16 \text { Driller's log } \\
\text { from } 16 \text { to } 3228 .\end{array}$ \\
\hline 1286 & $\begin{array}{l}\text { Tide Water Associ- } \\
\text { ated oil, Company }\end{array}$ & $\begin{array}{l}\text { Josie Parker } \\
\text { No. } 1\end{array}$ & $\begin{array}{l}\mathrm{sec} \cdot 33, \mathrm{~T} 7 \mathrm{~s}, \mathrm{R} 19 \mathrm{E}, \\
\mathrm{Lacros} \text {, I mi. } \\
\text { sW }\end{array}$ & $\begin{array}{l}158 \mathrm{Grd} \\
168 \mathrm{DF}\end{array}$ & 1947 & 3,220 & $\begin{array}{l}17 \text { samples from } 10 \\
\text { to } 170 \text { ft. No samples } \\
\text { from } 170 \text { to } 1675 \text { ft. } \\
159 \text { samples from } \\
1575 \text { to } 3219 \text { ft. } \\
\text { Cores from No. i } 3 \\
2850-2860 \text { to No. } 41 \\
\text { 3 } 32191-3220 \text { schlum } \\
\text { berger from } 145 \text { to } \\
3218 . \text { Driller's log } \\
\text { from o to } 3220 .\end{array}$ \\
\hline
\end{tabular}


BAKER COUNTY

\begin{tabular}{|c|c|c|c|c|c|c|c|}
\hline $\begin{array}{l}\text { FGS } \\
\text { NO. }\end{array}$ & COMPANY OY ONNERS & $\begin{array}{l}\text { FARi Or } \\
\text { WELL NAME }\end{array}$ & LOCATION & $\begin{array}{l}\text { ELEV- } \\
\text { ATION }\end{array}$ & $\begin{array}{l}\text { YEAR } \\
\text { COML- } \\
\text { PLETED }\end{array}$ & DEPTH & RERARKS \\
\hline in- & $\begin{array}{l}\text { Hunt Oil Company } \\
\text { Humble Oil \& Re- } \\
\text { fining Company }\end{array}$ & H.L.Hunt No.I & $\begin{array}{l}\text { Sec.21, TIN, R2OE, } \\
\text { Baxter, about } 5 \\
\text { mi. SW } \\
\text { Sec.36, T3S,R21E, } \\
\text { Manning, I mi.NE }\end{array}$ & $\begin{array}{l}124 \mathrm{Grd} \\
134 \mathrm{DF} \\
131 \mathrm{Grd}\end{array}$ & 1947 & 3,349 & $\begin{array}{l}306 \text { samples from } 10 \\
\text { to } 3343 \text { ft. Cores } \\
\text { from No. } 1 \text { \$ } 2880- \\
2890 \text { to No. } 19 \text { } 3348 \text { - } \\
3349 . \text { schlumberger } \\
\text { from } 67 \text { to } 3348 \text {. } \\
\text { Driller's log from } 0 \\
\text { to } 3349 . \\
\text { May 6, 1947, Location } \\
\text { approved. August } 20, \\
\text { l947 location aban- } \\
\text { doned. }\end{array}$ \\
\hline
\end{tabular}

BAY COUNTY

\begin{tabular}{|c|c|c|c|c|c|c|c|}
\hline 933 & $\begin{array}{l}\text { Magnolia Petroleum } \\
\text { Company }\end{array}$ & $\begin{array}{l}\text { State No.I, } \\
\text { Block } 4-B\end{array}$ & $\begin{array}{l}\text { seo.2l, T35, R15W, } \\
\text { Panama city, } \\
7 \mathrm{mi} . \mathrm{NW}\end{array}$ & $\begin{array}{ll}2.2 & \mathrm{Grd} \\
7.0 \mathrm{DF}\end{array}$ & 1945 & 7,003 & $\begin{array}{l}713 \text { samples from } 0 \text { to } \\
7000 \text { ft. Cores at in- } \\
\text { tervals from No.1 } 3 \\
4138-4148 \text { to No.16 } \\
6853-6865 \text {. No recov- } \\
\text { ery on some cores. } \\
\text { schlumberger from } 458 \\
\text { to } 7003 . \text { Driller's } \\
\text { log from o to } 7000 \text {. }\end{array}$ \\
\hline
\end{tabular}


BREVARD COUNTY

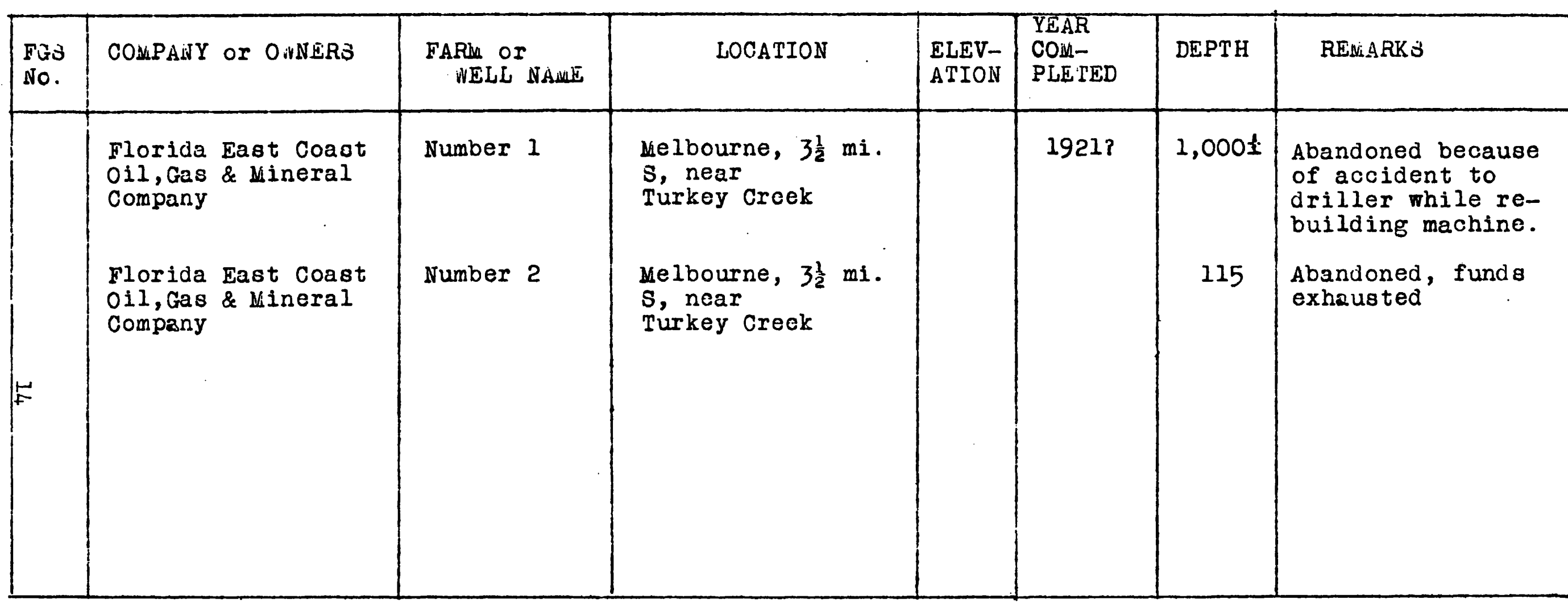

BROHARD COUNTY

\begin{tabular}{|c|c|c|c|c|c|c|c|}
\hline${ }^{W}-$ & $\begin{array}{l}\text { Port Everglades } \\
\text { Oil and Gas } \\
\text { Company }\end{array}$ & Number 1 & $\begin{array}{l}\text { sec. } 15, \mathrm{~T} 50 \mathrm{~s}, \mathrm{R} 4 \mathrm{EE}, \\
14 \mathrm{th} \text { st. SW be- } \\
\text { tween } 4 \mathrm{th} \& 6 \mathrm{th} \\
\text { Avenues } \\
\text { Fort Lauderdale }\end{array}$ & $8 \frac{1}{2} \div$ & 1929 & 3,010 & $\begin{array}{l}34 \text { samples from } \\
1,940 \text { to } 3005 \text { ft. } \\
\text { Driller's } 10 g \\
\text { from } 0 \text { to } 3000 \text {. }\end{array}$ \\
\hline
\end{tabular}


CALHOUN COUNTY

\begin{tabular}{|c|c|c|c|c|c|c|c|}
\hline $\begin{array}{l}\text { Fữ } \\
\text { No. }\end{array}$ & COMPANY OI ONNERS & $\begin{array}{l}\text { FAFüL or } \\
\text { NELL NAME }\end{array}$ & LOCATIOIN & $\begin{array}{l}\text { ELEV- } \\
\text { ATION }\end{array}$ & \begin{tabular}{|l|} 
YEAR \\
CONL- \\
PLETED \\
\end{tabular} & DEPTH & REMARKS \\
\hline$w-7$ & $\begin{array}{l}\text { Calhoun Gas \& Oil } \\
\text { Company }\end{array}$ & & $\begin{array}{l}\text { Sec. } 36, \text { TlN, Rlow, } \\
\text { clarksville, I/2 } \\
\text { mi. NW }\end{array}$ & $120 \pm$ & 1920 & 1,320 & $\begin{array}{l}101 \text { samples from } 10 \\
\text { to } 1320 \mathrm{ft} \text {. }\end{array}$ \\
\hline $\begin{array}{l}\text { W- } \\
1103\end{array}$ & Pure Oil Company & $\begin{array}{l}\text { St. Andrews } \\
\text { Bay Proper- } \\
\text { ties Co.No.I }\end{array}$ & $\begin{array}{l}\text { Sec. } 2, T 13, R 11 \pi, \\
\text { Blountstown, } 16 \\
\text { mi. W }\end{array}$ & $\begin{array}{l}183 \mathrm{Grd} \\
197 \mathrm{DF}\end{array}$ & 1945 & 3,580 & $\begin{array}{l}110 \text { samples from } 1 \text { to } \\
3555 \text { ft. scilumberger } \\
\text { from } 758 \text { to } 3578 \text {. } \\
\text { Driller's log } 0 \text { to } \\
3580 \text {. }\end{array}$ \\
\hline $\begin{array}{l}\text { W- } \\
1104\end{array}$ & Pure 011 Company & $\begin{array}{l}\text { St. Andrews } \\
\text { Bay Proper- } \\
\text { ties Co.NO.2 }\end{array}$ & $\begin{array}{l}\text { sec. } 25, T 1 N, R 11 W \\
\text { Blounts town, } 15 \\
\text { mi. W }\end{array}$ & $\begin{array}{l}172 \mathrm{Grd} \\
186 \mathrm{DF}\end{array}$ & 1945 & 4,457 & $\begin{array}{l}142 \text { samples from } 104 \\
\text { to } 4457 \text { ft. Schlum- } \\
\text { berger from } 217 \text { to } \\
4456 \text {. Driller's log } \\
\text { from o to } 4457 \text {. }\end{array}$ \\
\hline $\begin{array}{l}\mathrm{W}- \\
1101\end{array}$ & Pure Oil Company & $\begin{array}{l}\text { International } \\
\text { Paper Co. } \\
\text { No. } 1\end{array}$ & $\begin{array}{l}\text { Sec. } 25, T l N, R I I W \\
\text { Blountctown, ló } \\
\text { mi. SW }\end{array}$ & $\begin{array}{l}113 \mathrm{Grd} \\
127 \mathrm{DF}\end{array}$ & 1945 & 3,460 & $\begin{array}{l}113 \text { samples from } 1 \text { td } \\
3437 \text { ft. jchlumberger } \\
\text { from } 228 \text { to } 3457 \text {. } \\
\text { Driller's log from o } \\
\text { to } 3460 \text {. }\end{array}$ \\
\hline $\begin{array}{l}W- \\
1100\end{array}$ & Pure Oil Company & $\begin{array}{l}\text { International } \\
\text { Paper Co. } \\
\text { No. } 2\end{array}$ & $\begin{array}{l}\text { sec. } 31, \text { Tls, RlOW, } \\
\text { Blountstown, } 15 \\
\text { mi. W. }\end{array}$ & $\begin{array}{l}93 \mathrm{Grd} \\
107 \mathrm{DF}\end{array}$ & 1945 & 5,096 & $\begin{array}{l}165 \text { samples from } 14 \\
\text { to } 5093 \text { ft. Schlum- } \\
\text { berger from } 190 \text { to } \\
5094 \text {. Driller's log } \\
\text { from o to } 5096\end{array}$ \\
\hline 1099 & Pure 0 il Company & $\begin{array}{l}\text { International } \\
\text { Paper Co. } \\
\text { No. } 3\end{array}$ & $\begin{array}{l}\text { Sec. } 5, \text { Tls, Rlow, } \\
\text { Blountstown, } 12 \\
W\end{array}$ & $\begin{array}{l}146 \mathrm{Grd} \\
160 \mathrm{DF}\end{array}$ & 1945 & 3,040 & $\begin{array}{l}99 \text { samples from } 0 \text { to } \\
3015 \text { ft. Schlumberged } \\
\text { from } 232 \text { to } 3033 \text {. } \\
\text { Driller's log from o } \\
\text { to } 3040\end{array}$ \\
\hline
\end{tabular}




\section{CALHOUN COUNTY - Continued}

\begin{tabular}{|c|c|c|c|c|c|c|c|}
\hline $\begin{array}{l}\text { FGS } \\
\text { No. }\end{array}$ & COMPANY OY ONNERS & $\begin{array}{l}\text { FARN or } \\
\text { NELL NAME }\end{array}$ & LOCATION & $\begin{array}{l}\text { ELEV- } \\
\text { ATION }\end{array}$ & $\begin{array}{l}\text { YEAR } \\
\text { COM- } \\
\text { PLETED }\end{array}$ & DEPTH & RENIARKS \\
\hline $\begin{array}{l}W- \\
1510\end{array}$ & D.E.L.Byers & $\begin{array}{l}\text { Hardaway Con- } \\
\text { otruction Co. } \\
\text { No. } 1\end{array}$ & 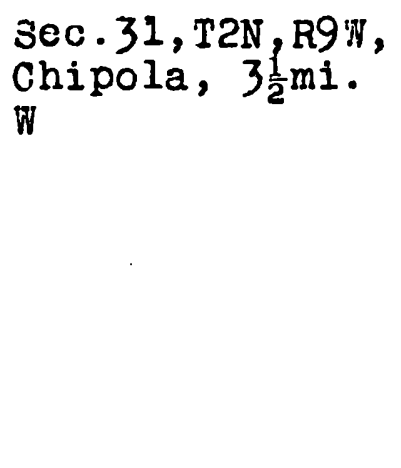 & $\begin{array}{l}217 \mathrm{Grd} \\
225 \mathrm{DF}\end{array}$ & 1947 & 4,873 & $\begin{array}{l}449 \text { samples from } 0 \\
\text { to } 4873 \text { ft. Cores } \\
\text { from IIo. } 1 \text { @ } 3377- \\
3397 \text { to No. } 4 \text { a } \\
4108-4128.3 c h l \text { lum- } \\
\text { berger from } 90 \text { to } \\
4876 \text {. Driller's } \\
\text { log from o to } 4873 \text {. } \\
\text { six side wall cores } \\
\text { from } 2549 \text { to } 4238\end{array}$ \\
\hline
\end{tabular}

CHARLOTTE COUNTY

\begin{tabular}{|c|c|c|c|c|c|c|c|}
\hline 979 & $\begin{array}{l}\text { Humble Oil \& Re- } \\
\text { fining Company }\end{array}$ & $\begin{array}{l}\text { Lowndes Tread- } \\
\text { well No. } 1\end{array}$ & $\begin{array}{l}\text { sec. } 17, \text { T42j, R23E, } \\
\text { Punta Gorda, } 6 \\
\text { mi. } \$\end{array}$ & $\begin{array}{l}20 \mathrm{DF} \\
\text { est. }\end{array}$ & 1945 & 13,300 & $\begin{array}{l}492 \text { samples from } \\
100 \text { to } 13,300 \text { ft. } \\
\text { Cores from No.1 } 0 \\
1807-1822 \text { to Core } \\
\text { No. } 158 \text { @ } 13295- \\
13300 . \text { with few } \\
\text { gaps. schlum- } \\
\text { berger from } 5558 \\
\text { to } 13304 \text {. Driller's } \\
\text { log from o to } \\
13300 \text {. }\end{array}$ \\
\hline
\end{tabular}


CITRUS COUINTY

\begin{tabular}{|c|c|c|c|c|c|c|c|}
\hline $\begin{array}{l}\text { Fũs } \\
\text { No. }\end{array}$ & COWPANY or OBNERS & $\begin{array}{l}\text { FARin or } \\
\text { WELI NAWE }\end{array}$ & LOSATION & $\begin{array}{l}\text { ELEV- } \\
\text { ATION }\end{array}$ & $\begin{array}{l}\text { YEAR } \\
\text { COSil- } \\
\text { PLETED }\end{array}$ & DEPTH & RENARKS \\
\hline $\begin{array}{l}\text { Wgi- } \\
207\end{array}$ & $\begin{array}{l}\text { Pedrson Oil } \\
\text { Company }\end{array}$ & & $\begin{array}{l}\text { Sec.9, Tl8s, Rl7E, } \\
\text { Crystal River, } \\
2 \text { mi. N }\end{array}$ & & 1905 & 1,900 & No samples or $\mathrm{log}$. \\
\hline
\end{tabular}

\section{CLAY COUNIY}

\begin{tabular}{|c|c|c|c|c|c|c|c|}
\hline 1590 & $\begin{array}{l}\text { Humble Oil \& Re- } \\
\text { fining Company }\end{array}$ & $\begin{array}{l}\text { Foremost Prop- } \\
\text { erties Corp. } \\
\text { No. } 1\end{array}$ & $\begin{array}{l}\text { sec. } 4, \text { TSS, R25E } \\
\text { Green Cove springs } \\
7 \mathrm{mi} .\end{array}$ & $\begin{array}{l}105 \mathrm{Grd} \\
115 \mathrm{DF}\end{array}$ & 1947 & 5,852 & $\begin{array}{l}172 \text { cuttings from } \\
40 \text { to } 5 \$ 50 \text { ft., } \\
\text { with some wide } \\
\text { gaps cores from } \\
\text { No. i } 727-732 \text { to } \\
\text { No. } 220 \text { \$ } 5859- \\
5862 \text { with } 14 \text { cores } \\
\text { missing. 3chlum- } \\
\text { berger from } 103 \text { to } \\
5861 \text { Driller's } \\
\text { log from } 100 \text { to } \\
5862 \text {. }\end{array}$ \\
\hline
\end{tabular}


GOLLIER COUNTY

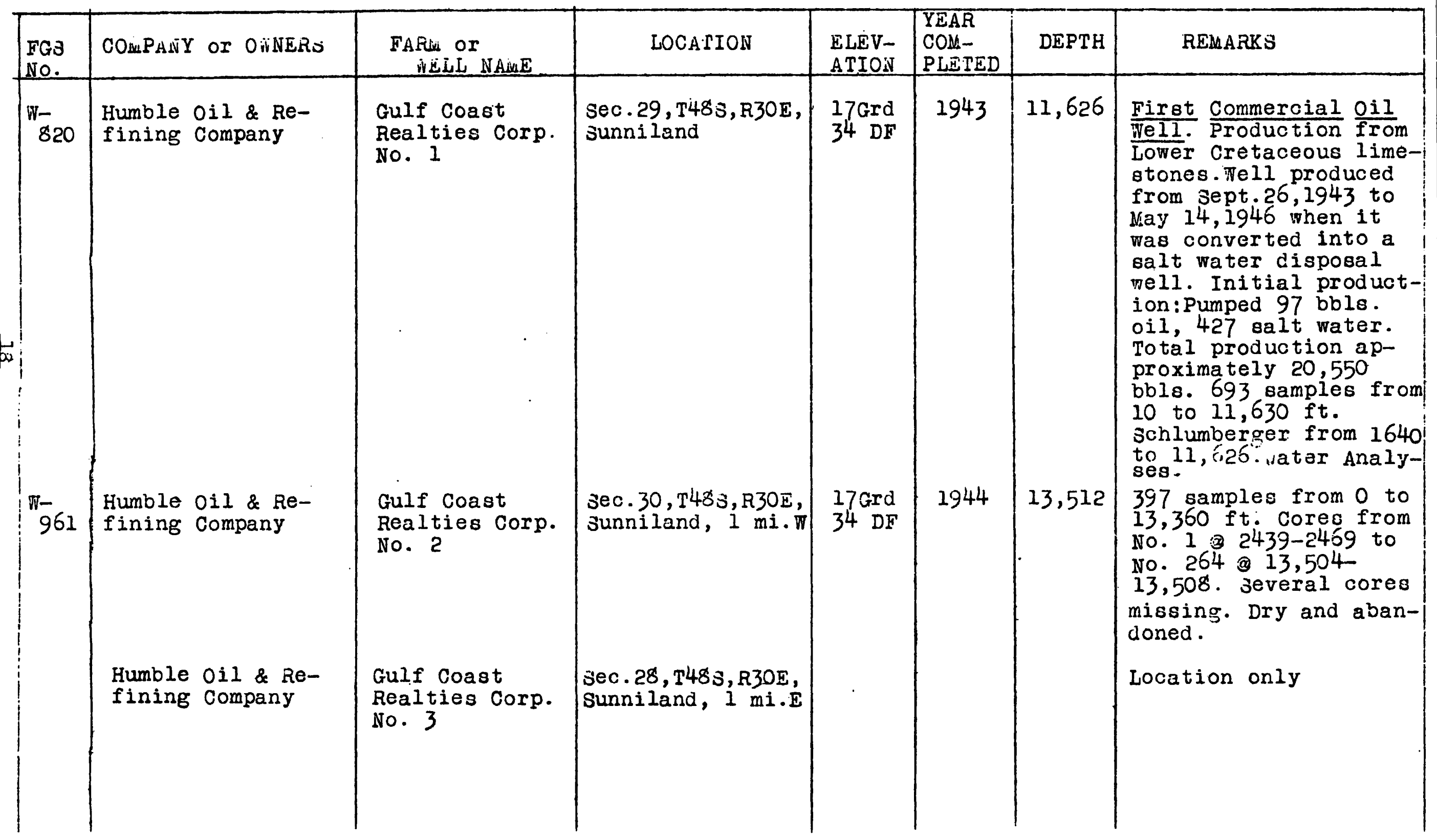


COLLIER COUNTY - Continued

\begin{tabular}{|c|c|c|c|c|c|c|c|}
\hline $\begin{array}{l}\text { FGS } \\
\text { NO. }\end{array}$ & COMPANY OY ONNERO & $\begin{array}{l}\text { FARW or } \\
\text { NELL NAME }\end{array}$ & LOCATION & $\begin{array}{l}\text { ELEV- } \\
\text { ATION }\end{array}$ & $\begin{array}{l}\text { YEAR } \\
\text { COL- } \\
\text { PLETED }\end{array}$ & DEPTH & RENARKS \\
\hline W- 949 & $\begin{array}{l}\text { Humble Oil \& Re- } \\
\text { fining Company }\end{array}$ & $\begin{array}{l}\text { Gulf Coast } \\
\text { Realties Corp. } \\
\text { No. } 4\end{array}$ & + & $\begin{array}{l}21 \mathrm{Grd} \\
34 \mathrm{DF}\end{array}$ & 1945 & 11,597 & $\begin{array}{l}\text { Second } \frac{\text { oil }}{\text { Pell }} \text {. } \\
\text { Pumping } \\
\text { June } 29,1945 \text { well } \\
\text { made } 495 \text { bbls. of } \\
\text { fluid of which } 327 \\
\text { bbls. were oil and } \\
158 \text { were water. } 228 \\
\text { samples from } 64 \text { to } \\
10,934 \text { ft. Core No. } \\
18 \text { @ } 6275-6285 \text { and } \\
5 \text { cores from 10,957 } \\
\text { to } 11,578 \text {. lany gaps } \\
\text { in. samples and cores. } \\
\text { schlumberger from } \\
1448 \text { to } 11,583\end{array}$ \\
\hline $\begin{array}{l}\text { W- } \\
1495\end{array}$ & $\begin{array}{l}\text { Humble Oil \& Re- } \\
\text { fining Company }\end{array}$ & $\begin{array}{l}\text { Gulf Coast } \\
\text { Realties Corp. } \\
\text { No. } 5\end{array}$ & $\begin{array}{l}\mathrm{Sec} \cdot 20, \mathrm{~T} 48 \mathrm{8}, \mathrm{R} 30 \mathrm{E}, \\
\text { sunniland, } 1_{\frac{1}{2}} \mathrm{mi} . \mathrm{N}\end{array}$ & $\begin{array}{l}18 \mathrm{Grd} \\
31 \mathrm{DF}\end{array}$ & 1947 & 11,578 & 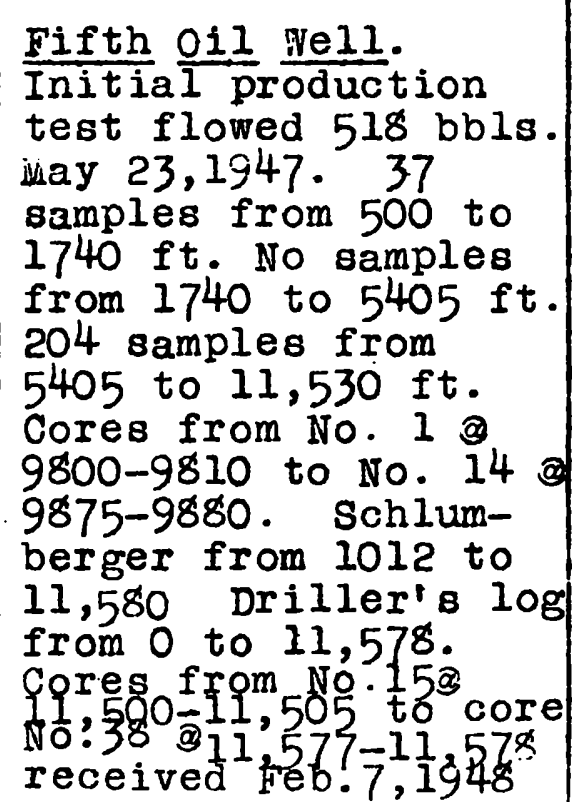 \\
\hline
\end{tabular}


COLLIER COUNTY - Continued

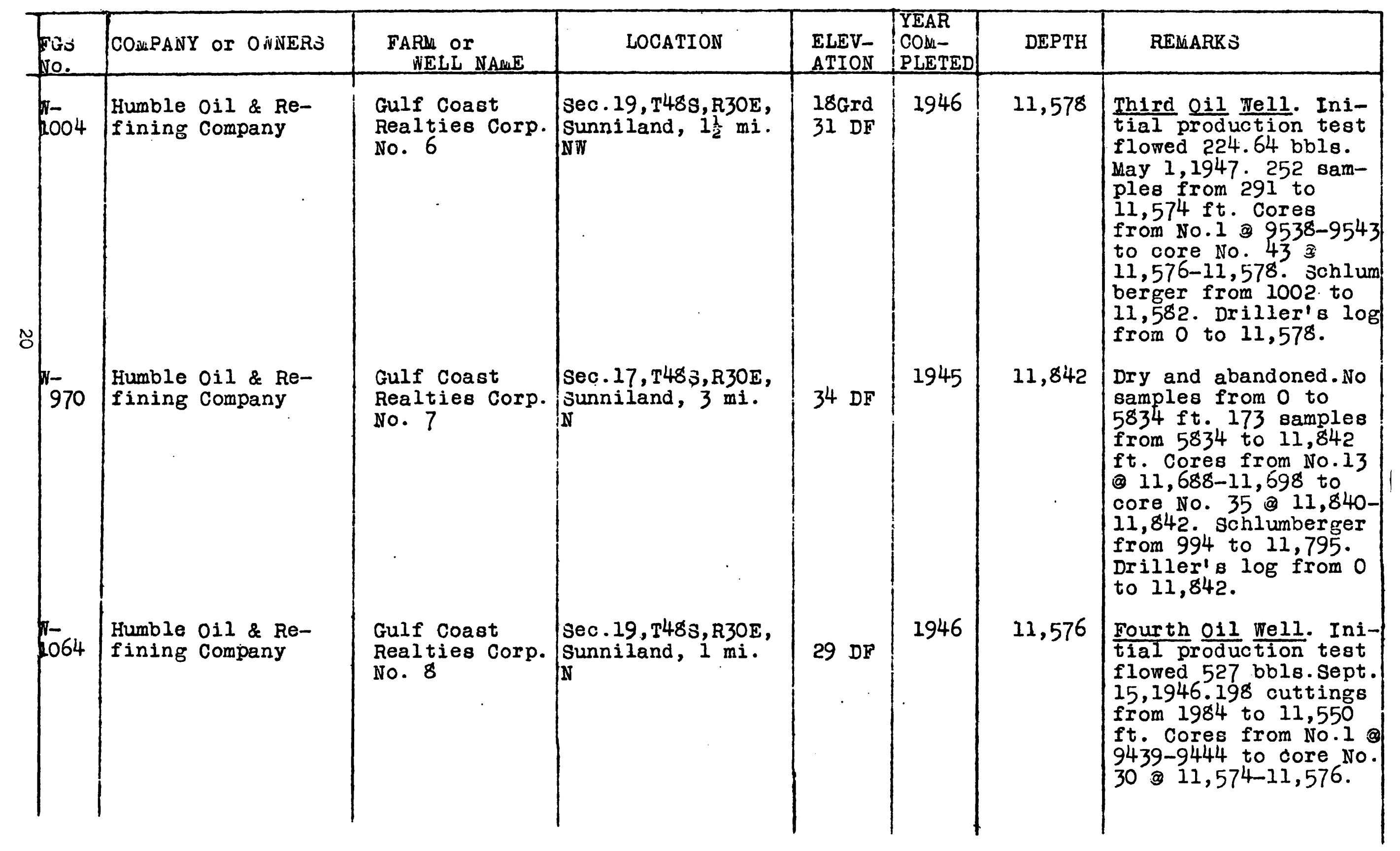


COLLIER COUNTY - Continued

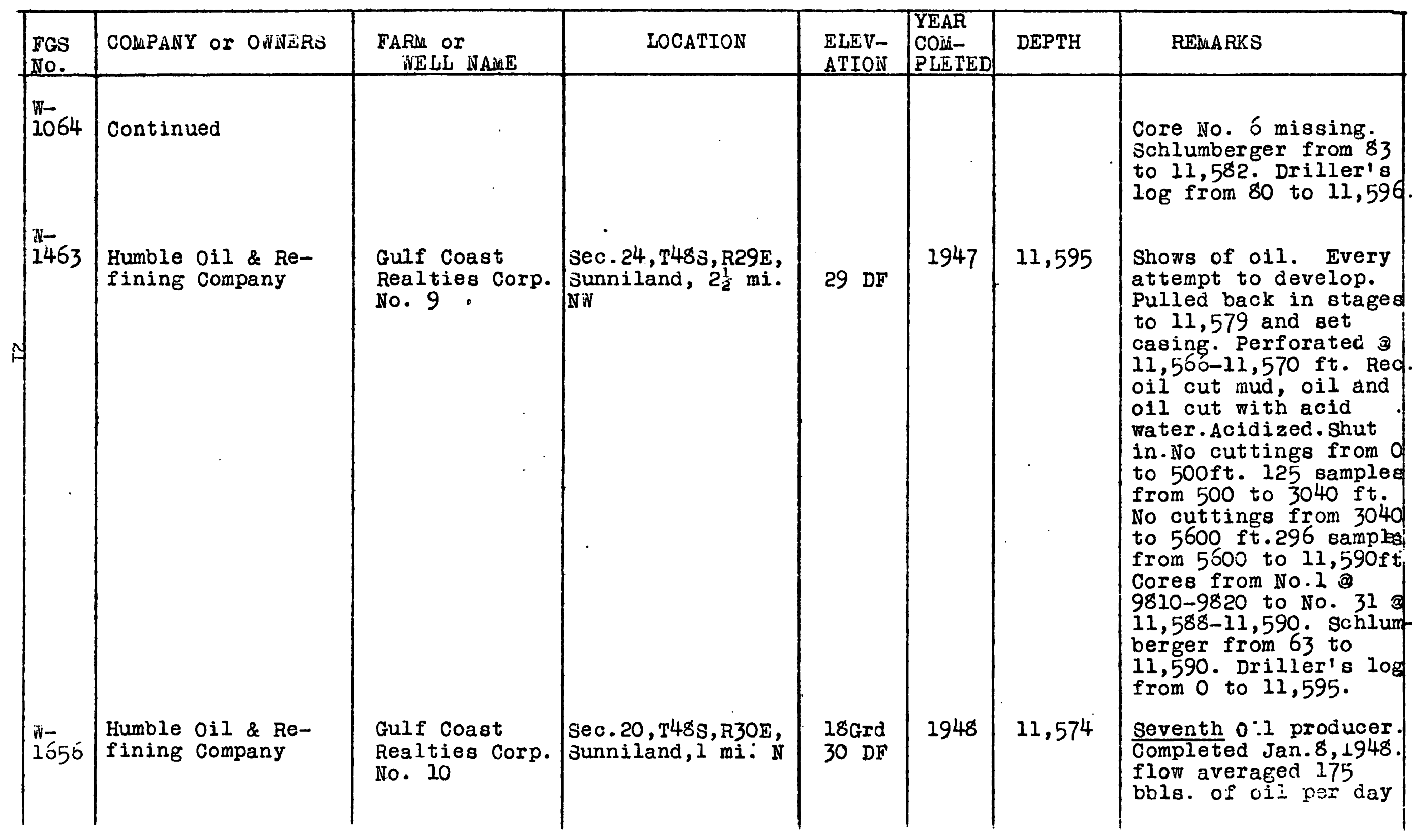


COLLIER COUNTY - Continued

\begin{tabular}{|c|c|c|c|c|c|c|c|}
\hline $\begin{array}{l}\text { FGS } \\
\text { No. }\end{array}$ & CONPANY or ONNER & $\begin{array}{l}\text { FARM or } \\
\text { NELLL NALWE }\end{array}$ & LOCATION & $\begin{array}{l}\text { ELEV- } \\
\text { ATION }\end{array}$ & $\begin{array}{l}\text { YEAR } \\
\text { COM- } \\
\text { PLETED } \\
\end{array}$ & DEPTH & REWARKS \\
\hline 1656 & Continued & & & & & & $\begin{array}{l}\text { on 1/4" choke. } 327 \\
\text { cuttings from o to } \\
11,560 \text { ft. } 19 \text { cores } \\
\text { from } 10 . \text { i } 11,390- \\
11,395 \text { to core No. } 20 \\
311,571-11,573 \text {. No } \\
\text { samples from } 3180 \text { to } \\
\text { 5110 ft. schlumberger } \\
\text { from } 84 \text { to } 11,582 \text {. } \\
\text { Driller's } 109 \text { from } \\
105 \text { to } 11,574 \text {. }\end{array}$ \\
\hline 1549. & $\begin{array}{l}\text { Humble oil \& Re- } \\
\text { fining Company }\end{array}$ & $\begin{array}{l}\text { Gulf Coast } \\
\text { Realties Corp. } \\
\text { No. Il }\end{array}$ & $\begin{array}{l}j \mathrm{Jec} .20, \mathrm{~T} 48 \mathrm{~S}, \mathrm{R} 30 \mathrm{E}, \\
\text { Junniland, id } \mathrm{mi} \\
\mathrm{NW}\end{array}$ & $\begin{array}{l}19 \mathrm{Grd} \\
30 \mathrm{DF}\end{array}$ & 1947 & 11,573 & $\begin{array}{l}\text { sixth oil producer. } \\
\text { Shows of oil. Swabbed, } \\
\text { acidized and reacid- } \\
\text { ized.Drill stem test } \\
\text { l1,550-11,573, open } \\
\text { tp-5/8n bottom choke, } \\
\text { open } 24 \text { hrs, flowed } \\
86 \text { bbis. oil. Oct.6, } \\
1947 \text { acidized with } \\
4000 \text { gls. Flowed } \\
160.24 \text { bblo. a day. } \\
\text { Completed oct.15, } \\
1947 \text { o } 120 \text { bbis. of } \\
24.50 \text { gravity oil } \\
\text { in } 24 \text { hrs. Nell flow- } \\
\text { ing. } 80 \text { cuttings from } \\
\text { loo to } 1760 \text { ft. No } \\
\text { returns from } 1760 \text { to } \\
5090 \text { ft. } 320 \text { cuttings }\end{array}$ \\
\hline
\end{tabular}


COLLIER COUNTY - Contjnued

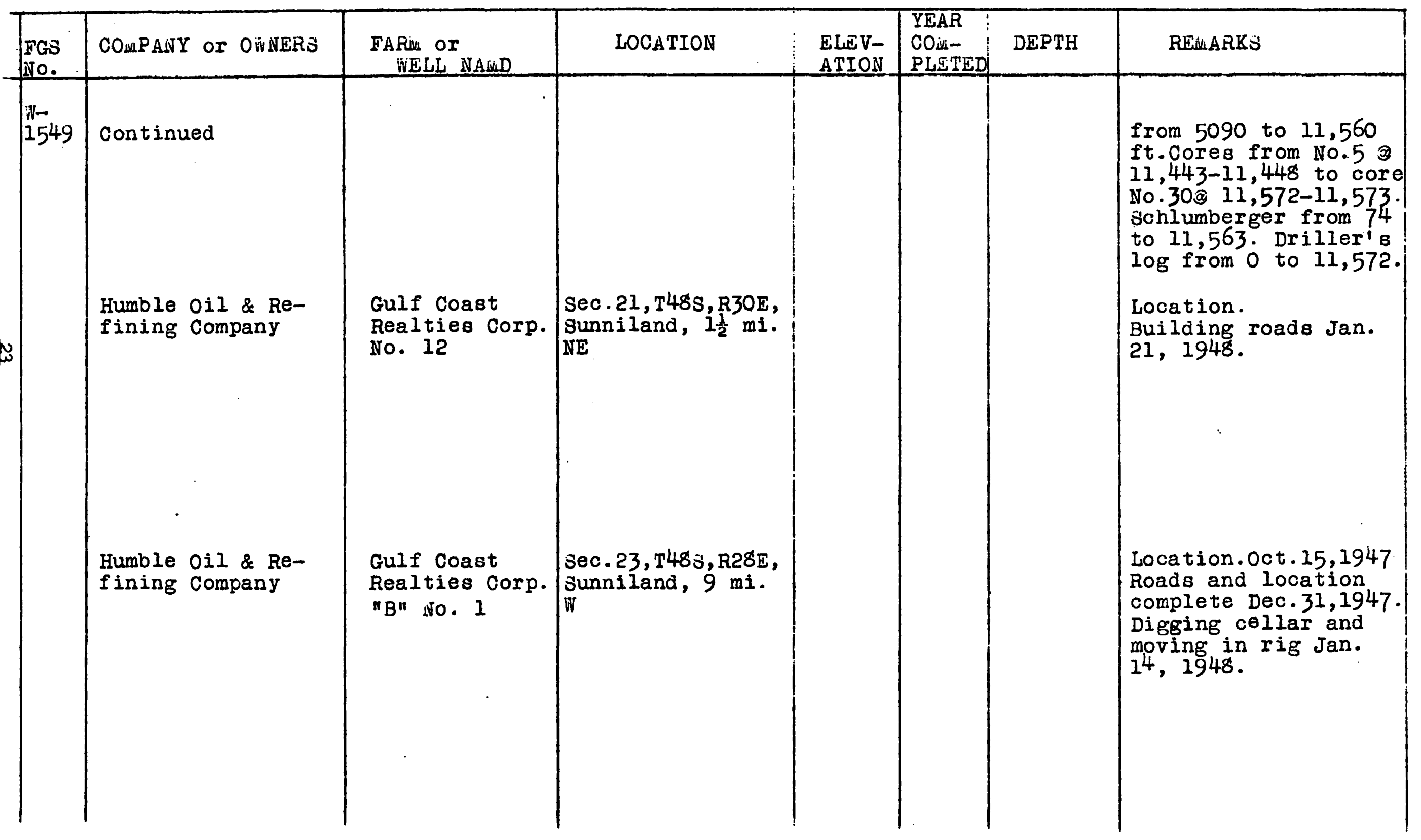


COLLIER COUNTY - Continued

\begin{tabular}{|c|c|c|c|c|c|c|c|}
\hline $\begin{array}{l}\text { FGS } \\
\text { No. }\end{array}$ & COLPANY Or ONNER & $\begin{array}{l}\text { FARR or } \\
\text { NELL NAWE }\end{array}$ & LOCATION & $\begin{array}{l}\text { QLEV } \\
\text { ATION }\end{array}$ & \begin{tabular}{|l|} 
YEAR \\
COML- \\
PLETED \\
\end{tabular} & DEPTH & REMARKS̈ \\
\hline Wgi- & $\begin{array}{l}\text { Humble Oil \& Re- } \\
\text { fining Company }\end{array}$ & $\begin{array}{l}\text { Lee Cypress } \\
\text { Co. No. } 1\end{array}$ & $\begin{array}{l}\text { Sec. } 28, \mathrm{~T} 47 \mathrm{~s}, \mathrm{R} 29 \mathrm{E}, \\
\text { Sunniland, } 9 \mathrm{mi} \text {. } \\
\text { NW }\end{array}$ & $\begin{array}{l}\text { 20Grd } \\
35 \text { DF }\end{array}$ & 1946 & 5,287 & $\begin{array}{l}\text { Lost drill pipe, well } \\
\text { abandoned. No samp- } \\
\text { les or cores. Moved } \\
35 \text { ft. to No. } 2 \text { lo- } \\
\text { cation. Schlumberger } \\
\text { from } 1014 \text { to } 4756 \text {. } \\
\text { Drillers log from o } \\
\text { to } 5287 .\end{array}$ \\
\hline 1484 & $\begin{array}{l}\text { Humble Oil \& Re- } \\
\text { fining Company }\end{array}$ & $\begin{array}{l}\text { Lee Cypress } \\
\text { Co. No. } 2\end{array}$ & $\begin{array}{l}\text { Skidded } 35^{\prime} \mathbf{E} \\
\text { of No: } 3 \\
\text { location }\end{array}$ & $\begin{array}{l}\text { 20Grd } \\
35 \mathrm{DF}\end{array}$ & 1946 & 1,175 & $\begin{array}{l}\text { Lost hole.Derrick } \\
\text { and rig moved to No. } \\
3 \text { location. } 29 \text { samp- } \\
\text { les from } 504 \text { to } 1080 \\
\text { ft. No 3chlunberger. } \\
\text { Driller's log from } \\
\text { to } 1175 \text {. }\end{array}$ \\
\hline 1485 & $\begin{array}{l}\text { Humble Oil \& Re- } \\
\text { fining Company }\end{array}$ & $\begin{array}{l}\text { Lee Cypress } \\
\text { Co. NO. } 3\end{array}$ & $\begin{array}{l}\text { Sec. } 28, T 473, \text { R29E, } \\
\text { about } 1250 ! \mathrm{E} \\
\text { from No. } 1\end{array}$ & $\begin{array}{l}19 \mathrm{Grd} \\
34 \mathrm{DF}\end{array}$ & 1947 & 11,943 & 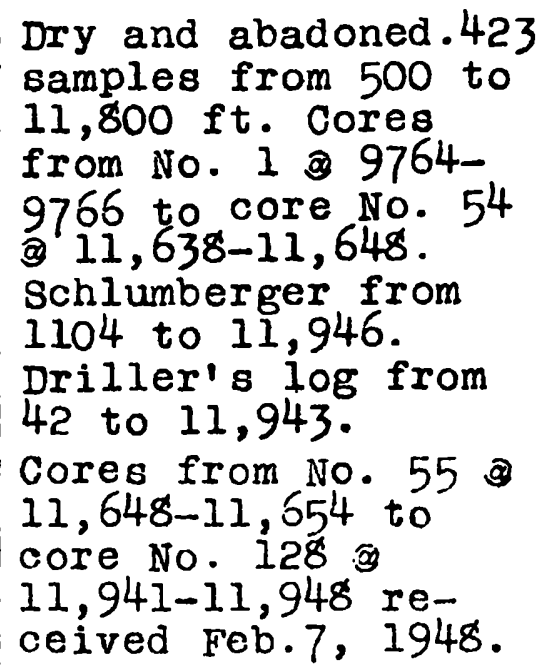 \\
\hline
\end{tabular}


Table 1

COmPLETION DATA ON HUMBLE OIL \& REFINING COMPANY' '̀ Gulf Coast Realties Corporation WELLS, SUNNILAND FIELD, COLLIER COUNTY, FLORIDA

\begin{tabular}{|c|c|c|c|c|c|c|c|c|c|c|}
\hline $\begin{array}{l}\text { Nidi } \\
\text { No. }\end{array}$ & $\begin{array}{l}\text { ELEV- } \\
\text { ATION }\end{array}$ & $\begin{array}{l}\text { TOTAL } \\
\text { DEPTH }\end{array}$ & $\begin{array}{l}\text { COMPLETION } \\
\text { DATE }\end{array}$ & $\begin{array}{l}\text { INITIAL } \\
\text { PRO- } \\
\text { DUCTION }\end{array}$ & CHOKE & $\begin{array}{l}\text { TUBING } \\
\text { PRESSURE }\end{array}$ & $\begin{array}{l}\text { CAOING } \\
\text { PRESOURE }\end{array}$ & $\begin{array}{l}\text { PRODUCING } \\
\text { ZONE }\end{array}$ & GRAVITY & $\begin{array}{l}\text { GAS } \\
\text { OIL } \\
\text { RATIO }\end{array}$ \\
\hline $\begin{array}{l}{ }^{*} \text { GCRC } \\
*_{\text {\#1 }}\end{array}$ & $34^{\prime} \mathrm{DF}$ & 11,626 & Nov. 26,1943 & $\begin{array}{l}97 \text { bbls } \\
\text { oil, } 425 \\
\text { bbls. } 3 \text {. }\end{array}$ & Pump & & & $\begin{array}{l}\text { open hole } \\
11,600- \\
11,626\end{array}$ & $\begin{array}{l}20.8 \\
\text { API }\end{array}$ & \\
\hline $\begin{array}{l}\text { GCRC } \\
\vec{\pi}^{2}\end{array}$ & $34^{\prime} \mathrm{DF}$ & 13,512 & Nov $.9,1944$ & $D \& A$ & & & & & & \\
\hline $\begin{array}{c}\text { GCRC } \\
\# 4\end{array}$ & $3^{\prime} \mathrm{DF}$ & 11,597 & Jun.24, 1945 & $257.34 \mathrm{~B} .0$. & Pump & & & $\begin{array}{l}\text { Open hole } \\
11,560- \\
11,597\end{array}$ & 19.2 & \\
\hline $\begin{array}{l}\text { GCRC } \\
\pi 55\end{array}$ & 311 & 11,578 & Wiay 24,1947 & $518 \mathrm{BOPD}$ & $1 / 4 n$ & 380 & 265 & $\begin{array}{l}\text { open hole } \\
11,560- \\
11,578\end{array}$ & 25 & 136 \\
\hline $\begin{array}{l}\text { GCRC } \\
\# 6\end{array}$ & 311 & 11,578 & May 1,1947 & $224.65 \mathrm{BOPD}$ & $5 / 3211$ & 350 & sealed & $\begin{array}{l}\text { open hole } \\
11,556- \\
11,578\end{array}$ & 25.6 & 67 \\
\hline $\begin{array}{l}\text { GCRC } \\
\# 7\end{array}$ & 341 & 11,842 & Oct.21, 1945 & $D \& A$ & & & & & & \\
\hline $\begin{array}{l}\text { GCRC } \\
\# 8\end{array}$ & $29^{\prime}$ & 11,576 & Sept 15, 1946 & 527 BOPD & $1 / 4 n$ & 350 & 410 & $\begin{array}{l}\text { Perfs } \\
11,554 \\
11,569\end{array}$ & 24.5 & 138 \\
\hline $\begin{array}{c}\text { GCRC } \\
\# 9\end{array}$ & 298 & 11,595 & SHUT IN PRE & P. TO ABAND & bN & & & & & \\
\hline \begin{tabular}{l} 
GCRC \\
\hdashline 10
\end{tabular} & $30^{\prime}$ & 11,574 & $\operatorname{Jan} .8,1948$ & 175 BOPD & $1 / 4 "$ & 93 & & $\begin{array}{l}\text { Open fole } \\
11,565- \\
11,574\end{array}$ & 24.8 & $100 ?$ \\
\hline $\begin{array}{l}\text { GCRC } \\
\text { ifII }\end{array}$ & $30^{\prime}$ & 11,573 & oct.14,1947| & $120 \mathrm{BOFD}$ & $1 / 2 "$ & 35 & 60 & $\begin{array}{l}\text { open hole } \\
11,550 \\
11,573\end{array}$ & 25.4 & 90 \\
\hline & & & & & & & & & & \\
\hline
\end{tabular}

*Abandoned as Prod. Niay 14, 1947 and completed as sil disposal well. 
Table 2

Production of Oil from Humble Oil \& Refining Company's Sunniland Field, Collier County, Florida, from September 1943 tinrough December 31, 1947.

\begin{tabular}{|c|c|c|c|c|c|}
\hline Month & 1943 & 1944 & 1945 & 1946 & 1947 \\
\hline Jan. & & 2,108 & - & 3,899 & 7,464 \\
\hline Feb. & & 1,404 & 402 & 3,454 & 5,203 \\
\hline Mar. & & 1,100 & 581 & 2,982 & 18,795 \\
\hline Apr. & & 1,054 & 403 & 4,151 & 16,362 \\
\hline May & & 1,115 & 725 & 6,776 & 20,440 \\
\hline June & & 822 & 4,110 & 7,677 & 31,065 \\
\hline July & & 1,123 & 5,700 & 6,450 & 31,395 \\
\hline Aug. & & 957 & 4,455 & 4,160 & 31,021 \\
\hline Sept. & $\begin{array}{l}\text { Discovery } \\
\text { Date Sept. } \\
26,1943\end{array}$ & 516 & 2,775 & 6,545 & 28,431 \\
\hline oct. & 648 & 609 & 2,976 & - & 22,445 \\
\hline Nov. & 643 & 581 & 2,666 & 2,407 & 23,948 \\
\hline Dec. & 2,741 & .449 & 2,717 & 8,384 & 22,776 \\
\hline $\begin{array}{l}\text { Total } \\
\text { Barrels }\end{array}$ & 4,032 & 11,838 & 27,510 & 56,885 & 259,345 \\
\hline $\begin{array}{l}\text { Total } \\
\text { cumulative } \\
\text { production } \\
\text { barrels }\end{array}$ & 4,032 & 15,870 & 43,380 & 100,265 & 359,610 \\
\hline
\end{tabular}


FLORIDA'S ONLY PRODUCITG FIELD AND NEARBY PROSPECTS IN COLLIER COUNTY.

R $23 E$

RQSE

R3OE

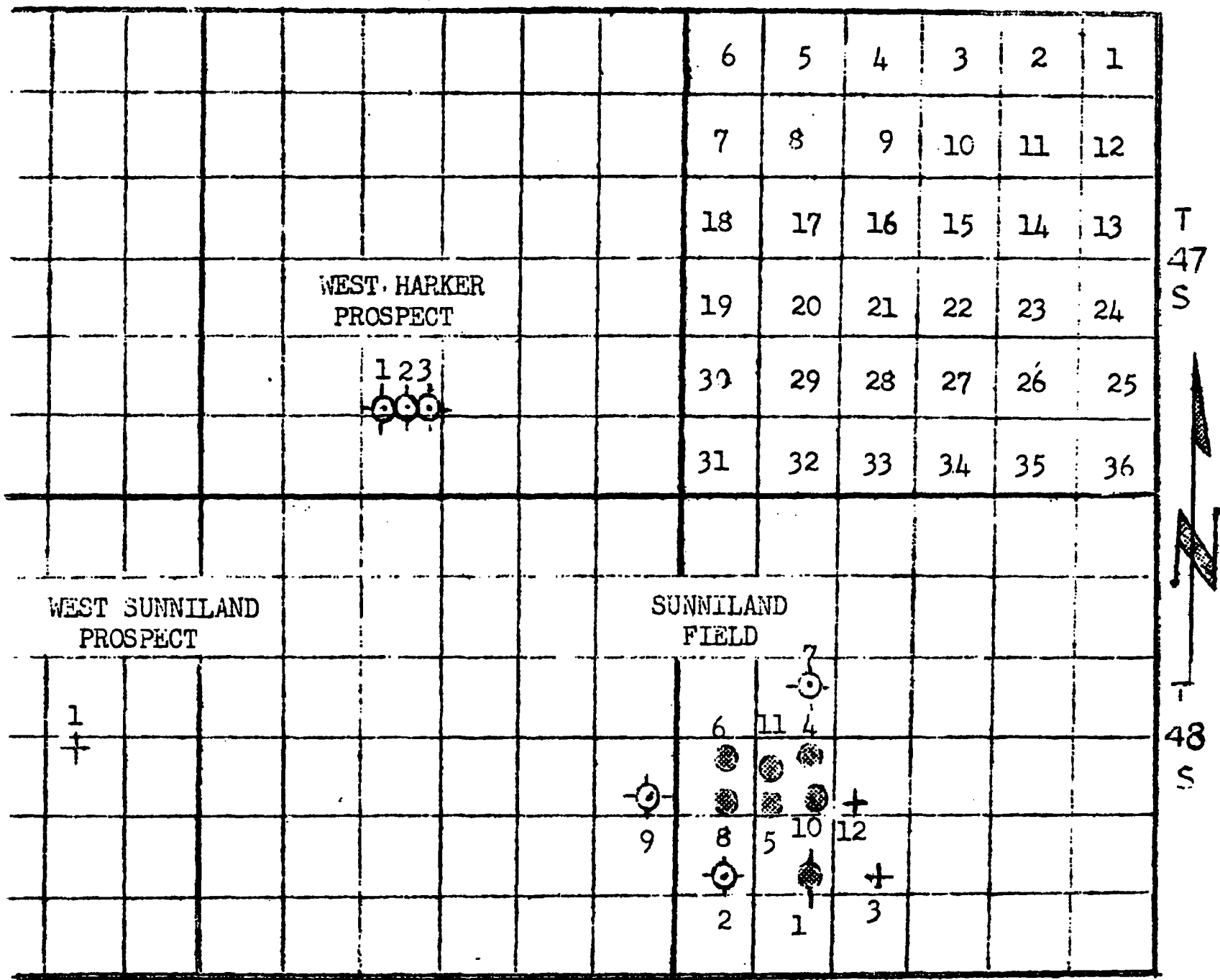

SUNNILAND FIELD - Farm Name: Gulf Coast Realties Corporation

1 - First cil producing well.Pumped.Producing horizon $11,600-11,626 \mathrm{ft}$. Produced Nov.26,1943 to May 15,1946.Converted into brine disposal well.

2 - Dry and abandoned at 13,512 $\mathrm{ft}$.

3 - Location only.

4 - Second oil producing well.Pumped.Producing horizon 11,560-11,597 ft.

5 - Fifth oil producing well. Flows. Producing horizon $11,560-11,578 \mathrm{ft}$.

6 - Third producing well, first flowing. Producing horizon $11,556-11,579 \mathrm{ft}$.

7 - Dry and abandoned at $11,842 \mathrm{ft}$.

8 - Fourth oil producing well. Flows. Producing horizon 11,564-11,569 ft.

9 - Shows of oil. Total depth $11,595 \mathrm{ft}$. Shut in, preparing to abandon.

10 - Seventh oil producing well. Flows. Producing horizon 11, 566-11, $574 \mathrm{ft}$.

11 - Sixth oil producing well. Flows. Producing horizon 11,550-11,573 ft.

12 - Location, January 12, 1948.

WEST HARKER PROSPECT - Farm Name: Lee Cypress Company

1 - Lost drill pipe. Total depth 5,287 ft. Skidded to location No. 2,

2 - Lost hole. Total depth $1,175 \mathrm{ft}$. Moved to location No. 3.

3 - Dry and abandoned at $11,943 \mathrm{ft}$.

WEST SUNNILAND PROSPECT - Farm Name: Gulf Coast Realties Corporation "B"

1 - Location. Ruads completed Dec,31,1947. Noving in rig Jan. 14,1948. 


\section{COLUmBIA COUNTY}

\begin{tabular}{|c|c|c|c|c|c|c|c|}
\hline $\begin{array}{l}\text { FGS } \\
\text { NO. }\end{array}$ & COMPANY Or OWNERS & $\begin{array}{l}\text { FARRi or } \\
\text { \#ELL NAME }\end{array}$ & LOCATION & $\begin{array}{l}\text { ELEV- } \\
\text { ATION }\end{array}$ & $\begin{array}{l}\text { YEAR } \\
\text { COM- } \\
\text { PLETED }\end{array}$ & DEPTH & REViARKS \\
\hline$\pi n-21$ & $\begin{array}{l}\text { Suwannee oil \& } \\
\text { Development Co. }\end{array}$ & Lowe No. 1 & $\begin{array}{l}\mathrm{sec}, 26, \mathrm{~T} 3 \mathrm{~S}, \mathrm{Rl6E}, \\
\text { Lake City, } 4 \mathrm{mi} \text {. } \\
\text { W }\end{array}$ & & 1923 & 1,320 & $\begin{array}{l}\text { lo samples from } 675 \\
\text { to } 1320 \mathrm{ft} \text {. }\end{array}$ \\
\hline
\end{tabular}

\begin{tabular}{|c|c|c|c|c|c|c|c|}
\hline \multicolumn{8}{|c|}{ COUNTY } \\
\hline & $\begin{array}{l}\text { 3arver and } \\
\text { Snavely }\end{array}$ & & Hialeah, $2 \frac{1}{2} \mathrm{mi} . \mathbb{N}$ & & 1924 & & No reliable data \\
\hline & $\begin{array}{l}\text { Florida Petroleum } \\
\text { Exploration Co. }\end{array}$ & $\begin{array}{l}\text { Tatum Bros. } \\
\text { No. } 1\end{array}$ & $\begin{array}{l}\text { Block } 16 \text { in sec. } \\
6, \text { T58 3,R39E. } \\
\text { Florida City, } 6 \\
\text { mi. SE }\end{array}$ & & 1929 & $2,080 \pm$ & No record \\
\hline$\sqrt{n-}$ & $\begin{array}{l}\text { Miami Florida oil } \\
\text { and Gas Company }\end{array}$ & $\begin{array}{l}\text { Everglades } \\
\text { No. } 1\end{array}$ & $\begin{array}{l}\text { Sec. } 19, \text { T54s, R35E, } \\
\text { Miami, } 40 \text { mi. N } \\
\text { on Tamiami Trail }\end{array}$ & $14 \pm$ & 1932 & 4,570 & $\begin{array}{l}89 \text { cuttings from } 2600 \\
\text { to } 4570 \text { ft. l2 cores } \\
\text { at intervais from } \\
3099 \text { to } 4540 \text { ft. } \\
\text { Driller's log from o } \\
\text { to } 4560 \text { ft. Sample } \\
\text { log from } 3095 \text { to } 4570\end{array}$ \\
\hline${ }^{N-}$ & $\begin{array}{l}\text { Wm.G.Blanchard \& } \\
\text { Associates }\end{array}$ & $\begin{array}{l}\text { Everglades } \\
\text { No. } 1\end{array}$ & $\begin{array}{l}\text { Sec. } 31, T 533, R 35 E, \\
\text { Miami, } 43 \text { mi. } \mathbb{N} \\
\text { on Tamiami Trail }\end{array}$ & 9 & 1944 & 10,284 & $\begin{array}{l}\text { Shut down since July } \\
4,1944 \text {. At } 1270 \mathrm{ft} . \\
\text { gas was encountered, }\end{array}$ \\
\hline
\end{tabular}




\begin{tabular}{|c|c|c|c|c|c|c|c|}
\hline $\begin{array}{l}\text { FGS } \\
\text { NO. }\end{array}$ & COWPANY OF OWNERS & $\begin{array}{l}\text { FARM Or } \\
\text { TiELL NAME }\end{array}$ & LOCATION & $\begin{array}{l}\text { ELEV- } \\
\text { ATION }\end{array}$ & $\begin{array}{l}\text { YEAR } \\
\text { COIM- } \\
\text { PLETED }\end{array}$ & DEPTH & REHIARKS \\
\hline 935 & 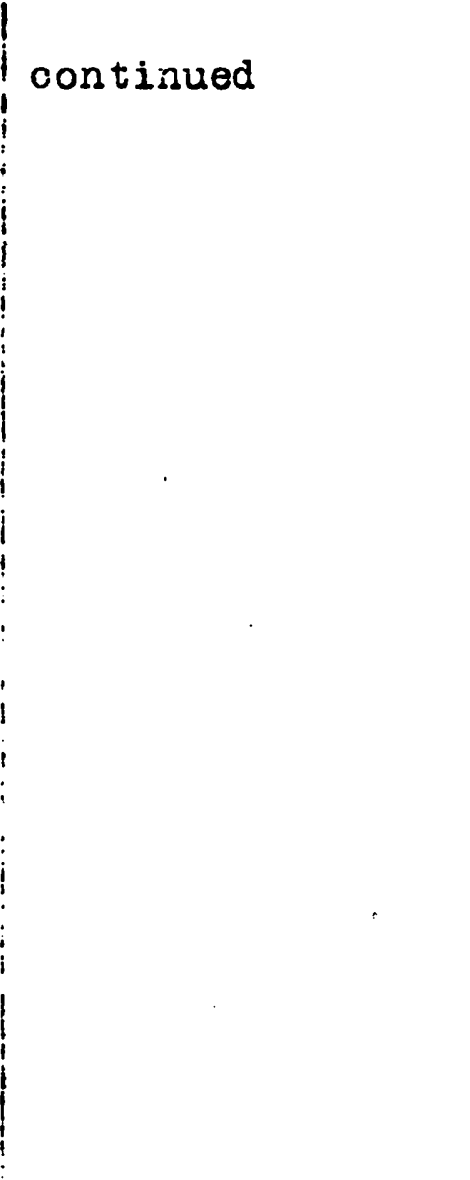 & & & & & & 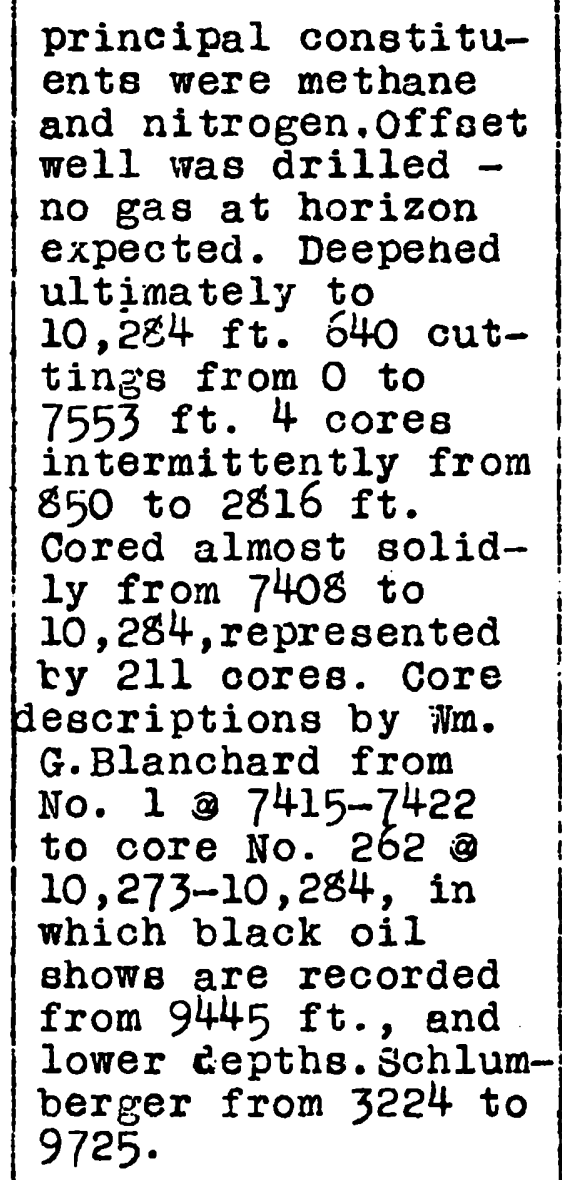 \\
\hline W66 & $\begin{array}{l}\text { Wm.G.Blanchard \& } \\
\text { Associates } \\
\end{array}$ & $\begin{array}{l}\text { Everglades } \\
\text { No. } 2\end{array}$ & $\begin{array}{l}\text { Sec. } 31, \text { T } 53, \mathrm{R} 35 \mathrm{E}, \\
\text { Wiami, } 33 \text { mi. on } \\
\text { Tamiami Trail. } \\
\text { few feet from No. } 1\end{array}$ & 9 & 1941 & 1,280 & $\begin{array}{l}\text { Drilled as an offset } \\
\text { to test if gas hori- } \\
\text { zon in No. 1 at } 1270 \\
\text { ft.was present. Not } \\
\text { encountered and well }\end{array}$ \\
\hline
\end{tabular}


DADE COUNTY - Continued

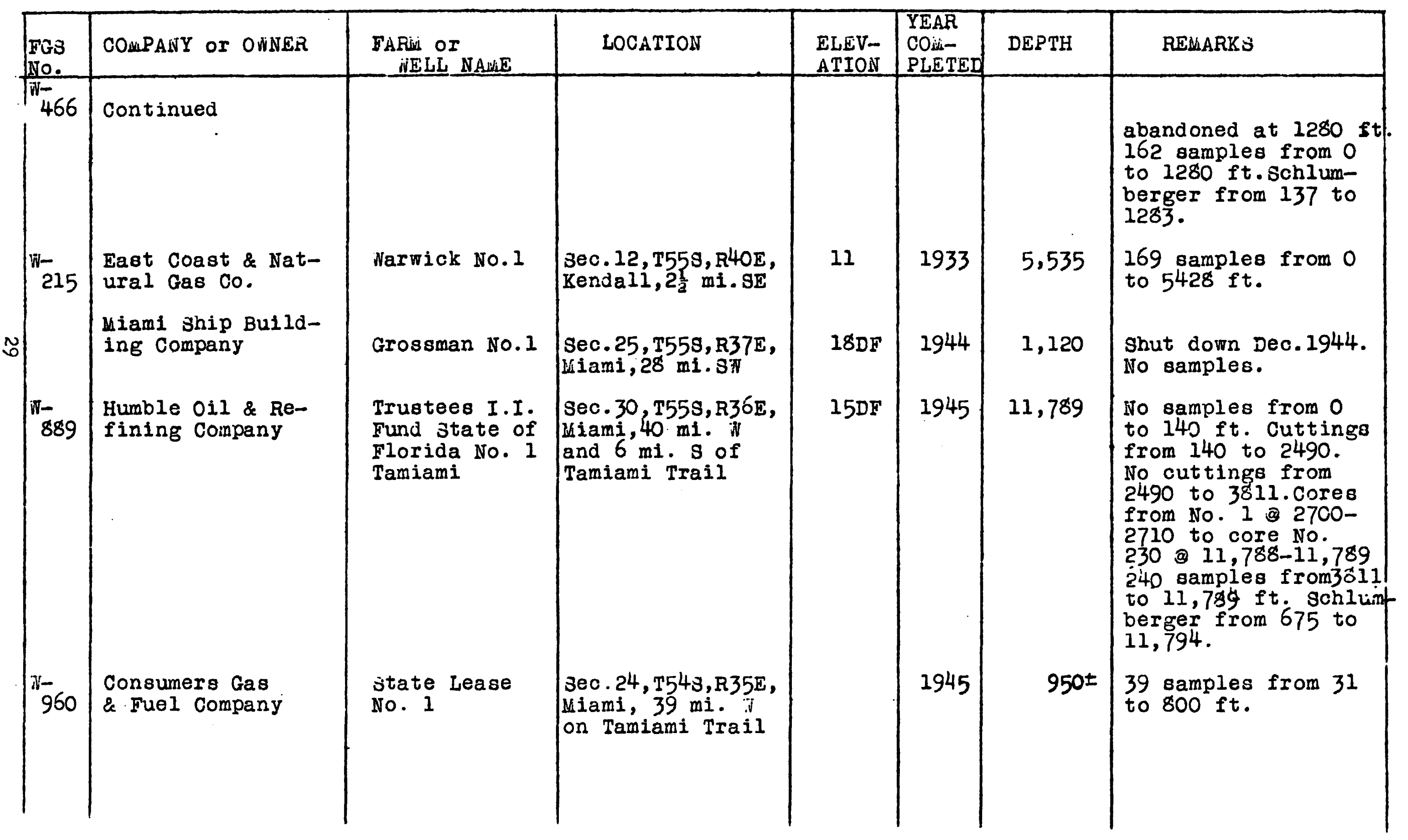


DADE COUNTY - Continued

\begin{tabular}{|c|c|c|c|c|c|c|c|}
\hline $\begin{array}{l}\text { FGS } \\
\text { NO. }\end{array}$ & COMPANY OT ONINERS & $\begin{array}{l}\text { FARin OI } \\
\text { VIVELL NAwE }\end{array}$ & LOCATION & $\begin{array}{l}\text { ELEV- } \\
\text { ATION }\end{array}$ & \begin{tabular}{|l|} 
YEAR \\
CONA- \\
PLETED \\
\end{tabular} & DEPTH & RE:BARKS̈ \\
\hline & O.D.Robinson & $\begin{array}{l}\text { O.D. Robinson } \\
\text { No. } 1 \text { Wodel } \\
\text { Land Company }\end{array}$ & $\begin{array}{l}\text { Jec. } 29, \text { T59s, R39E, } \\
\text { Homestead, about } \\
14 \text { mi. s on U.s. } \\
\text { Hwy. \#1 }\end{array}$ & & & & $\begin{array}{l}\text { Location in } 1946 . \\
\text { Abandoned. }\end{array}$ \\
\hline 1115 & Gulf oil Corp. & $\begin{array}{l}\text { No.l state } \\
\text { Model Land } \\
\text { Company "C" }\end{array}$ & $\begin{array}{l}\text { sec. ( unsurveyed) } \\
\text { T60s, R35E, } \\
\text { Homestead, about } \\
25-30 \mathrm{mi} . \mathrm{sil}\end{array}$ & $\begin{array}{l}1 \mathrm{Grd} \\
11 \mathrm{DF}\end{array}$ & 1946 & 6,030 & $\begin{array}{l}426 \text { cuttings from } 230 \\
\text { to } 5910 \text { ft. Cores from } \\
\text { No. } 1 \text { 3.6000-6010 to } \\
\text { core No.3 } 96020-6030 . \\
\text { Schlumberger from } \\
2975 \text { to } 6024 . \text { Drill- } \\
\text { er's log from } 12 \text { to } \\
6030 \text {. }\end{array}$ \\
\hline
\end{tabular}

DIXIE COUNTY

\begin{tabular}{|c|c|c|c|c|c|c|c|}
\hline 636 & $\begin{array}{l}\text { Florida Oil Devel- } \\
\text { opment Company }\end{array}$ & $\begin{array}{l}\text { Putnam Lumber } \\
\text { Company No. } 1\end{array}$ & $\begin{array}{l}\text { Sec. } 7, \text { TIIs, RI } \\
\text { Cross City, } 6 \mathrm{mi} . \\
\text { sW }\end{array}$ & $\begin{array}{l}18 \mathrm{Grd} \\
25 \mathrm{DF}\end{array}$ & 1942 & 4,780 & $\begin{array}{l}215 \text { samples from } 93 \\
\text { to } 4732 \text { ft. Schlumber- } \\
\text { ger from } 510 \text { to } 3860 \text {. }\end{array}$ \\
\hline 1124 & $\begin{array}{l}\text { Stanolind oil \& } \\
\text { Gas Company }\end{array}$ & $\begin{array}{l}\text { Perpetual For- } \\
\text { ests Inc., } \\
\text { No. } 1\end{array}$ & $\begin{array}{l}\operatorname{Sec} \cdot 5, \text { TIls, RIIE, } \\
\text { Cross City, } 8 \mathrm{mi} . \\
\text { Sil }\end{array}$ & $23 \mathrm{Grd}$ & 1946 & 7,510 & $\begin{array}{l}58 \text { samples from } 40 \text { to } \\
780 \text { ft. No samples } \\
\text { from } 780 \text { to } 1710 \text { ft. } \\
\text { lost circulation, ex- } \\
\text { cept } 1 \text { sample } 31650 \text {. } \\
567 \text { samples from } 1710 \\
\text { to } 7510 . \text { Cores from } \\
\text { No. } 1 \text { @ } 1942-1962 \text { to } \\
\text { core INo. } 77 \text { o } 7500-\end{array}$ \\
\hline
\end{tabular}


DIXIE COUNTY - Continued

\begin{tabular}{|c|c|c|c|c|c|c|c|}
\hline $\begin{array}{l}\text { FGS } \\
\text { NO. }\end{array}$ & CORHANY OI OWNER & $\begin{array}{l}\text { FARM or } \\
\text { WELL NAME }\end{array}$ & LOCATION & $\begin{array}{l}\text { ELEV- } \\
\text { ATION }\end{array}$ & $\begin{array}{l}\text { YEAR } \\
\text { COM- } \\
\text { PLETED } \\
\end{array}$ & DEPTH & REMARKS \\
\hline $\begin{array}{l}\text { W- } \\
1114\end{array}$ & Continued & & & & & & $\begin{array}{l}7510 \text {, few missing. } \\
\text { Also core descrip- } \\
\text { tions. schlumberger } \\
\text { from } 210 \text { to } 7506 \text {. } \\
\text { Driller's log from } 0 \\
\text { to } 7510 \text {. }\end{array}$ \\
\hline W- & Sun Oil Company & $\begin{array}{l}\text { Hazel Langs- } \\
\text { ton No. } 1\end{array}$ & $\begin{array}{l}\text { sec.8, TSS, RI4E, } \\
\text { Cross City, } 17 \\
\text { mi.NE }\end{array}$ & $\begin{array}{l}25 \mathrm{Grd} \\
33 \mathrm{DF}\end{array}$ & 1946 & 3,671. & $\begin{array}{l}243 \text { samples from } 41 \\
\text { to } 3671 \text { ft. Lost cir- } \\
\text { culation from 106 to } \\
1400 \text { ft., no samples. } \\
\text { Cores from No. } 1 \text {. } \\
1400-1410 \text { to core No. } \\
14 \text { 3668-3671, ex- } \\
\text { cept No. } 12-\text { no re- } \\
\text { covery. schlumberger } \\
\text { from } 57 \text { to } 3647 \text {. } \\
\text { Driller's log from o } \\
\text { to } 3671 \text {. Also core } \\
\text { description, water } \\
\text { analyois and other } \\
\text { data. }\end{array}$ \\
\hline
\end{tabular}


DUVAL COUNTY

\begin{tabular}{|c|c|c|c|c|c|c|c|}
\hline $\begin{array}{l}\text { FGS } \\
\text { INO. }\end{array}$ & COLLPAINY Or OHINER & $\begin{array}{l}\text { FARin or } \\
\text { NELL NAME }\end{array}$ & LOCATION & $\begin{array}{l}\text { ELEV- } \\
\text { ATION }\end{array}$ & $\begin{array}{l}\text { YEAR } \\
\text { COM- } \\
\text { PLETED }\end{array}$ & DEPTH & REMARKS \\
\hline & $\begin{array}{l}\text { East Coast } 011 \\
\text { Company }\end{array}$ & & $\begin{array}{l}\text { Sec. 12, T33, R25E, } \\
\text { Jacksonvilie, } 6 \\
\text { mi. SN }\end{array}$ & & 1923 & 1,690 & $\begin{array}{l}\text { No samples or log. } \\
\text { information very } \\
\text { meager }\end{array}$ \\
\hline
\end{tabular}

\section{EJCAHBIA COUNTY}

\begin{tabular}{|c|c|c|c|c|c|c|}
\hline $\begin{array}{l}\text { Pensacola Devel- } \\
\text { opment Company }\end{array}$ & Well No. 1 & $\begin{array}{l}\text { Pensacola, about } \\
5 \text { mi. N. in New } \\
\text { Warrington, west } \\
\text { side of Barrancas } \\
\text { Ave. \& south of } \\
\text { Gulf Beach Hwy.; } \\
\text { near junction. }\end{array}$ & & 1901 & 1,702 & $\begin{array}{l}\text { Driller'a log,USGS } \\
\text { iater-supply Paper } \\
\text { lo2, pp. } 257-258 .\end{array}$ \\
\hline $\begin{array}{l}\text { Pensacola Deve 1- } \\
\text { opment Company }\end{array}$ & rell No. 2 & $\begin{array}{l}\text { Pensacola, on } \\
\text { East Hill, inter- } \\
\text { section of loth } \\
\text { Ave. \& Mallory st }\end{array}$ & 70 & 1901 & 1,620 & $\begin{array}{l}\text { Driller's log, UsGS } \\
\text { Nater-supply Paper } \\
\text { lo2,pp.258-259,also } \\
\text { analysis of water, } \\
\text { very salty } 5,320 \text { ppm } \\
\text { salt. Log also in } \\
\text { I4th \& } 17 \text { th Ann. Repts } \\
\text { Fla.Geol. Survey. }\end{array}$ \\
\hline
\end{tabular}


ESCAMBIA COUNTY - Continued

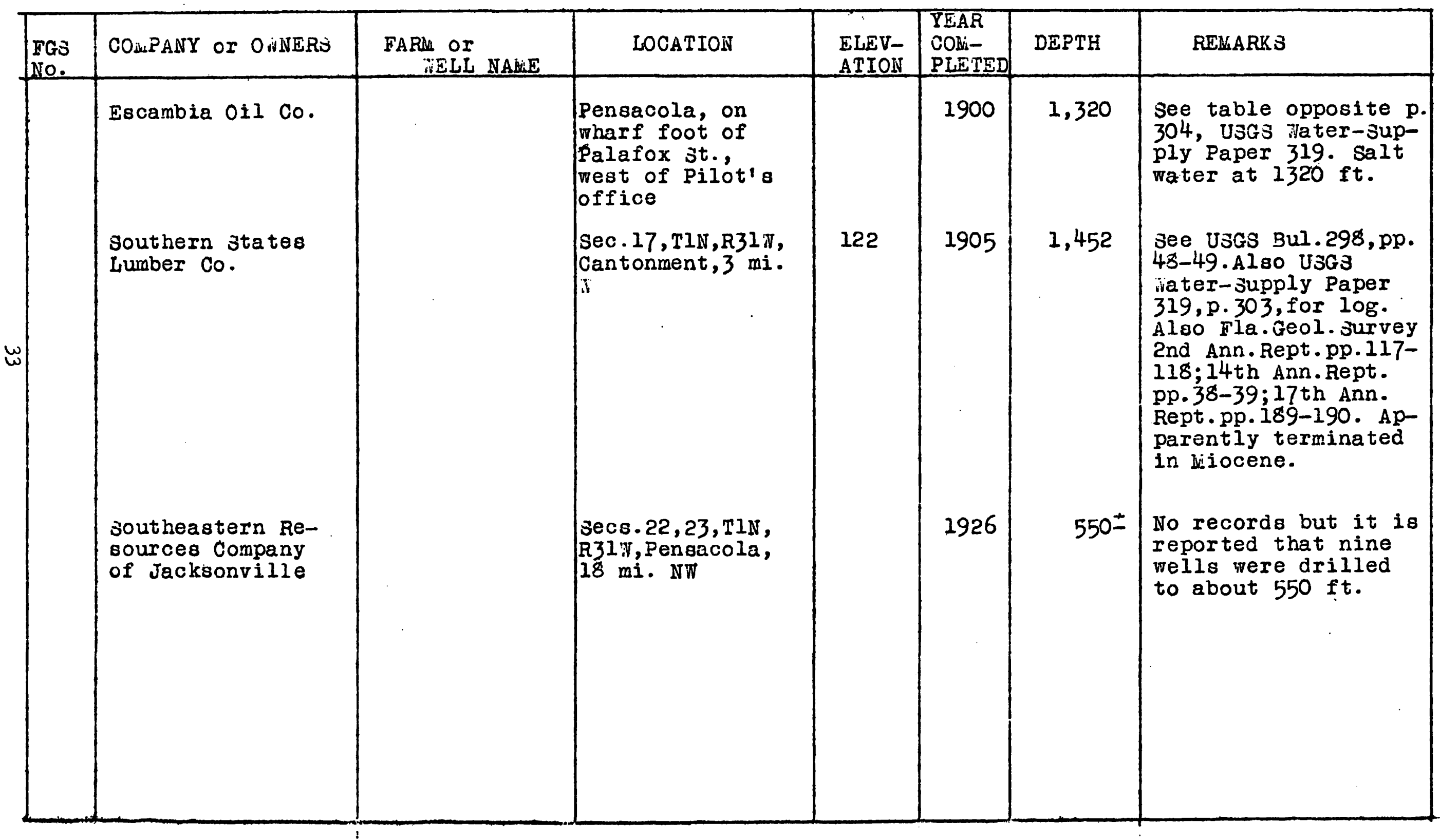


FLAGLER COUNTY

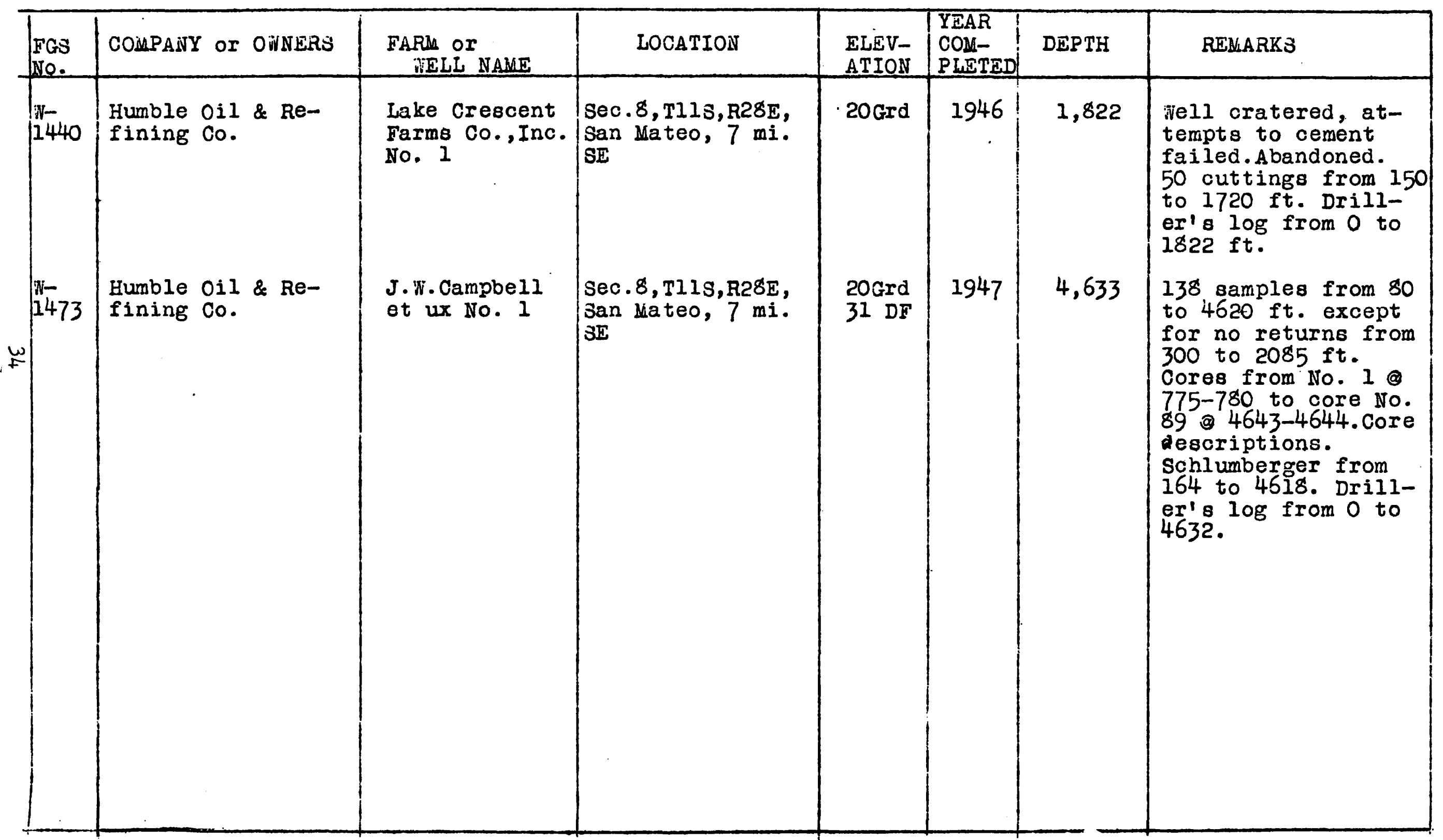


FRANKLIIN COUNTY - Continued

\begin{tabular}{|c|c|c|c|c|c|c|c|}
\hline $\begin{array}{l}\text { FGS } \\
\text { NO. }\end{array}$ & COMPAINY Or OWNERS & $\begin{array}{l}\text { FARUi Or } \\
\text { inELL NANAE }\end{array}$ & LOCATION & $\begin{array}{l}\text { ELEV- } \\
\text { ATION }\end{array}$ & $\begin{array}{l}\text { YEAR } \\
\text { CON- } \\
\text { PLETED } \\
\end{array}$ & DEPTH & REMARKS \\
\hline 1502 & $\begin{array}{l}\text { Magnolia Petroleum } \\
\text { Company. }\end{array}$ & $\begin{array}{l}\text { Florida state } \\
\text { Block 5-B } \\
\text { No. 1-A }\end{array}$ & $\begin{array}{l}\text { sec. } 23 ?, \text { T9s, } \\
\text { In st. Vincent } \\
\text { sound }\end{array}$ & $10 \mathrm{DF}$ & 1947 & 7,019 & $\begin{array}{l}639 \text { samples from } 320 \\
\text { to } 7010 \text { ft.Cores } \\
\text { from No.1 } \$ 3490- \\
3504 \text { to core No. } 24 \\
\text { @ } 7002-7019 \text {. Schlum- } \\
\text { berger from } 1649 \text { to } \\
7021 . \text { Driller's log } \\
\text { from 0 to } 7019 \text {. }\end{array}$ \\
\hline
\end{tabular}

$\omega$

\section{GADSDEN COUNTY}

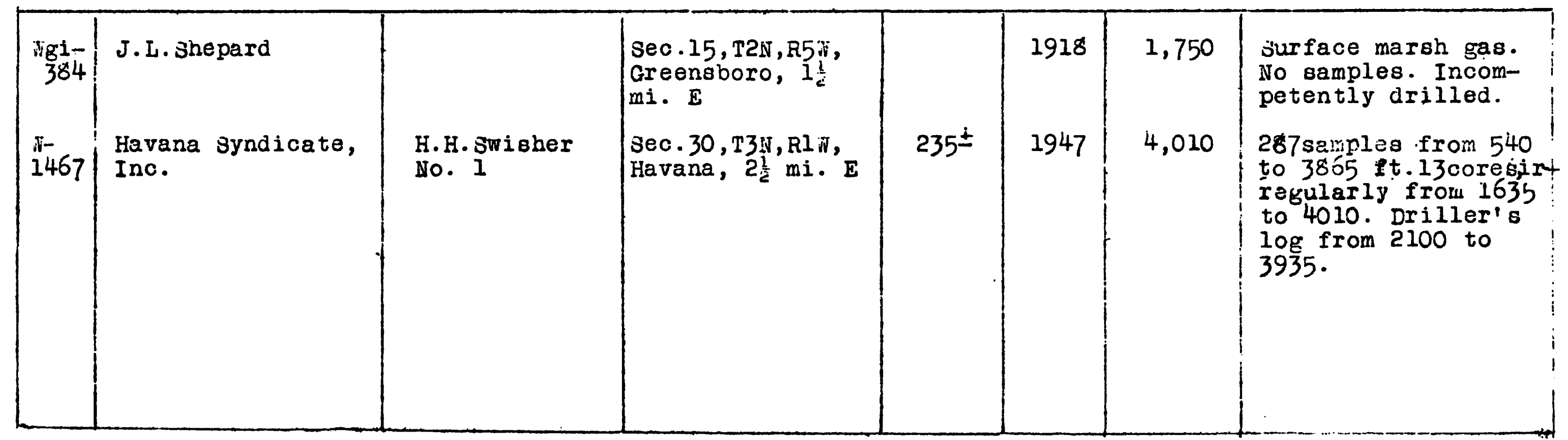


GILCHRIST COUNTY

\begin{tabular}{|c|c|c|c|c|c|c|c|}
\hline $\begin{array}{l}\text { FGS } \\
\text { Ne. }\end{array}$ & COHPANY OI ONNERS & $\begin{array}{l}\text { FARM or } \\
\text { OIELL NAME }\end{array}$ & LOCATION & $\begin{array}{l}\text { ELEV- } \\
\text { ATION }\end{array}$ & \begin{tabular}{|l|} 
YEAR \\
COM- \\
PLETED \\
\end{tabular} & DEPTH & REMARKS \\
\hline 1003 & sun Oil Company & $\begin{array}{l}\text { Alto Adams et } \\
\text { al No. } 1\end{array}$ & $\begin{array}{l}\text { Sec. } 15, T 9 s, R 32 \mathrm{E}, \\
\text { Trenton, } 6 \mathrm{mi} . \mathrm{N}\end{array}$ & $\begin{array}{l}88 \mathrm{Grd} \\
93 \mathrm{DF}\end{array}$ & 1946 & 3,753 & $\begin{array}{l}232 \text { cuttings from } 80 \\
\text { to } 3750 \text { ft.Cores from } \\
\text { No.1 } 33282-3289 \text { to } \\
\text { core No. } 43 \text { 9 } 3748- \\
3753 \text {. Schlumberger } \\
\text { from } 71 \text { to } 3748 \text {. } \\
\text { Driller's log from o } \\
\text { to } 3753 \text {. Core and } \\
\text { time records. }\end{array}$ \\
\hline
\end{tabular}

GLADES COUNTY

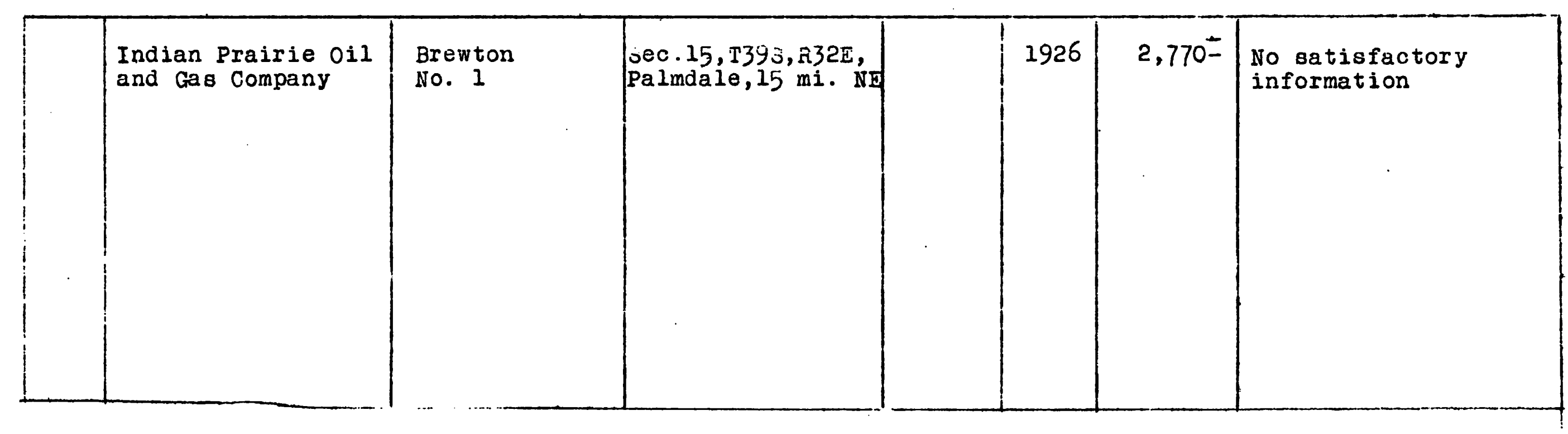


GULF COUNTY

\begin{tabular}{|c|c|c|c|c|c|c|c|}
\hline $\begin{array}{l}\text { FGS } \\
\text { No. }\end{array}$ & COUPANY Or OWNER & $\begin{array}{l}\text { FARM or } \\
\text { NELL NAME }\end{array}$ & LOCATION & $\begin{array}{l}\text { ELEV- } \\
\text { ATION }\end{array}$ & $\begin{array}{l}\text { YEAR } \\
\text { COM- } \\
\text { PLETED } \\
\end{array}$ & DEPTH & REMARXS \\
\hline $\begin{array}{l}n- \\
914\end{array}$ & Pure Oil Company & $\begin{array}{l}\text { C.C. Hopkins } \\
\text { No. } 1\end{array}$ & $\begin{array}{l}\text { Sec. } 22, T 6 s, R 9 \pi, \\
\text { Port st. Joe, } \\
14 \mathrm{mi} \text {. NE }\end{array}$ & $\begin{array}{l}18 \mathrm{Grd} \\
32 \mathrm{DF}\end{array}$ & 1944 & 8,708 & $\begin{array}{l}272 \text { cuttings from } 261 \\
\text { to } 8678 \text { ft.Cores from } \\
\text { No. } 131980-1997 \text { to } \\
\text { No. } 2398589-8708, \\
\text { with cores } 9,10,11 \\
\text { missing. 3chiumberger } \\
\text { from } 252 \text { to } 8713 \text {. }\end{array}$ \\
\hline 1470 & Pure Oil Company & $\begin{array}{l}\text { C.C.Hopkins } \\
\text { No. } 2\end{array}$ & $\begin{array}{l}\text { jec. } 21, \text { Tós, R9ij, } \\
\text { Port st. Joe, } \\
13 \mathrm{mi} \text {. NE }\end{array}$ & $\begin{array}{l}19 \text { Grd } \\
33 \mathrm{DF}\end{array}$ & 1945 & 7,255 & $\begin{array}{l}236 \text { cuttings from } 88 \\
\text { to } 7233 \text { ft. schlum- } \\
\text { berger from } 1902 \text { to } \\
7258 \text {. }\end{array}$ \\
\hline 1469 & Pure Oil Company & $\begin{array}{l}\text { Kate Gaskins } \\
\text { No. } 1\end{array}$ & $\begin{array}{l}\text { sec.19, T5s, R9i, } \\
\text { Port st. Joe, is } \\
\text { mi. NE }\end{array}$ & $\begin{array}{l}29 \text { srd } \\
43 \text { DF }\end{array}$ & 1945 & 5,606 & $\begin{array}{l}182 \text { cuttings from } 100 \\
\text { to } 5605 \text { ft. schlum- } \\
\text { berger from } 1965 \text { to } \\
5505 .\end{array}$ \\
\hline 1098 & Pure oil Company & $\begin{array}{l}\text { st.Joe Paper } \\
\text { Co. No. } 1\end{array}$ & $\begin{array}{l}\text { Jec } 10, \mathrm{~T} 7 \mathrm{~S}, \mathrm{R} 9 \mathrm{i}, \\
\text { Port st. Joe, } \\
12 \mathrm{mi} \text {. NE }\end{array}$ & $\begin{array}{r}7 \mathrm{Grd} \\
21 \mathrm{DF}\end{array}$ & 1945 & 5,796 & $\begin{array}{l}176 \text { cuttings from } 1 \\
\text { to } 5796 \text { ft. schlum- } \\
\text { berger from } 1510 \text { to } \\
5794 \text {. Driller's log } \\
\text { from } 0 \text { to } 5796 \text {. }\end{array}$ \\
\hline 1462 & Pure Oil Company & $\begin{array}{l}\text { St. Joe Paper } \\
\text { Co. No. } 3 \text { (for } \\
\text { Ho. } 2 \text { see } \\
\text { Franklin Coun- } \\
\text { ty }\end{array}$ & $\begin{array}{l}\text { sec.3,T6s,Rlifi, } \\
\text { Overstreet, } 3 \\
\text { mi. E }\end{array}$ & $\begin{array}{l}11 \mathrm{Grd} \\
25 \mathrm{DF}\end{array}$ & 1947 & 5,025 & $\begin{array}{l}165 \text { cuttings from } 15 \\
\text { to } 5009 \text { ft. Schlum- } \\
\text { berger from } 373 \text { to } \\
5023 \text {. Driller's log } \\
\text { from o to } 5025 \text {. }\end{array}$ \\
\hline 2455 & Pure Oil Company & $\begin{array}{l}\text { Pick Holling- } \\
\text { ex et al No. I }\end{array}$ & $\begin{array}{l}\text { jec.12, T9s, R117, } \\
\text { Port st. Joe, } 7 \\
\text { mi. s }\end{array}$ & $\begin{array}{r}5 \mathrm{Grd} \\
19 \mathrm{DF}\end{array}$ & 1946 & 5,656 & $\begin{array}{l}185 \text { cattinge : Irom } 50 \\
\text { to } 5616 \text { ft.schlum- } \\
\text { berger from } 447 \text { to }\end{array}$ \\
\hline
\end{tabular}




\section{GULF OOUNTY - Continued}

\begin{tabular}{|c|c|c|c|c|c|c|c|}
\hline $\begin{array}{l}\text { FGS } \\
\text { No. }\end{array}$ & COMLPAIY Or ONINERS & $\begin{array}{l}\text { FARM Or } \\
\text { WELL NANE }\end{array}$ & LOCA TION & $\begin{array}{l}\text { ELEV- } \\
\text { ATION }\end{array}$ & $\begin{array}{l}\text { YEAR } \\
\text { CON- } \\
\text { PLETED } \\
\end{array}$ & DEPTH & REMARKS \\
\hline 1455 & Continued & & & & & & $\begin{array}{l}5655 . \text { Driller's log } \\
\text { from o to } 5656 \text {. }\end{array}$ \\
\hline $\begin{array}{l}3- \\
1468\end{array}$ & Pure oil Company & $\begin{array}{l}\text { E.L. MCMillan } \\
\text { No. } 1\end{array}$ & $\begin{array}{l}\text { Sec. } 25, T 43, \text { Rl } 1 \text {, } \\
\text { iewahitchka, } 6 \\
\text { mi. i }\end{array}$ & $\begin{array}{l}35 \mathrm{Grd} \\
49 \mathrm{DF}\end{array}$ & 1947 & 5,069 & $\begin{array}{l}165 \text { cuttings froin } 47 \\
\text { to } 5059 \text { ft. Schlum- } \\
\text { berger frum } 263 \text { to } \\
5069 \text {. Driller's log } \\
\text { from o to } 5069 .\end{array}$ \\
\hline
\end{tabular}

HARDEE COUNTY

\begin{tabular}{|c|c|c|c|c|c|c|c|}
\hline 1655 & $\begin{array}{l}\text { Humble oil \& Re- } \\
\text { fining Co. }\end{array}$ & $\begin{array}{l}\text { B.T.Keen et } \\
\text { ux No. I }\end{array}$ & 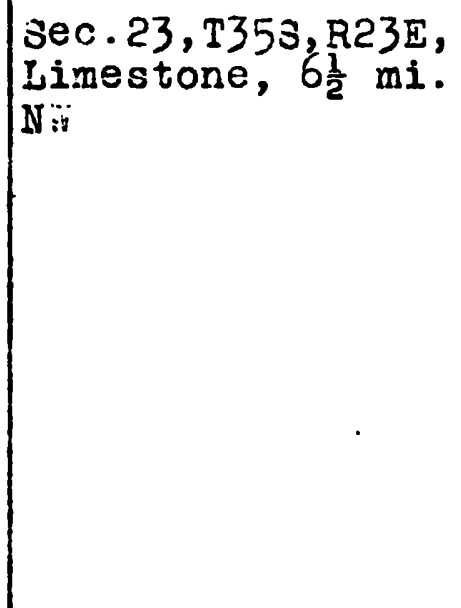 & $83 \mathrm{DF}$ & 1948 & 11,933 & 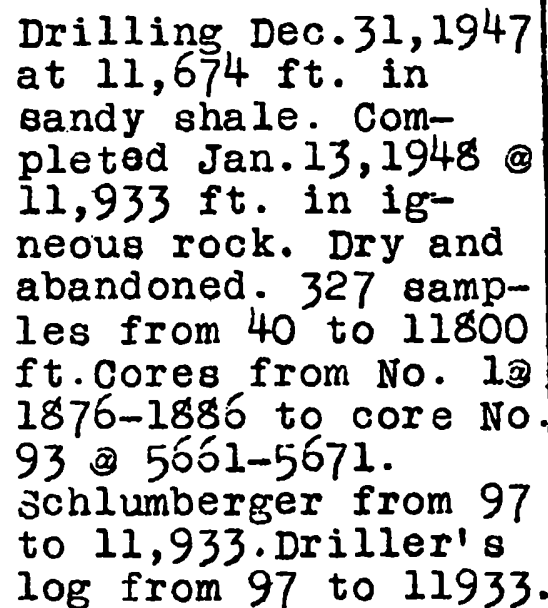 \\
\hline
\end{tabular}




\section{HIGHLANDS' COUiNTY - Continued}

\begin{tabular}{|c|c|c|c|c|c|c|c|}
\hline $\begin{array}{l}\text { FGS } \\
\text { No. }\end{array}$ & COMPAHY Or OVINERS & $\begin{array}{l}\text { FAFER or } \\
\text { WELL NALUE }\end{array}$ & LOCATION & $\begin{array}{l}\text { ELEV- } \\
\text { ATION }\end{array}$ & $\begin{array}{l}\text { YEAR } \\
\text { COML- } \\
\text { PLETED }\end{array}$ & DEPTH & RELARKS \\
\hline$\frac{\pi}{966}$ & Continued & & & & & & $\begin{array}{l}\text { from No. } 2 \text { @ } 1550- \\
1567 \text { to vore No.379 } \\
\text { \$ } 12,982-12,985 \text {, with } \\
\text { many gaps. Schlum- } \\
\text { berger from } 536 \text { to } \\
12,655 \text {. Driller's } \\
\text { log from o to } 12,985\end{array}$ \\
\hline
\end{tabular}

\section{HILLSBOROUGH COUNTY}

\begin{tabular}{|c|c|c|c|c|c|c|c|}
\hline$i-8$ & R.V.Hill & $\begin{array}{l}\text { R.E. OIds } \\
\text { No. I? }\end{array}$ & $\begin{array}{l}\text { Sec. } 18, \text { T28s, R172, } \\
\text { old smar, near }\end{array}$ & $10=$ & 1926 & 3,289 & $\begin{array}{l}125 \text { samples from } 400 \\
\text { to } 3185 \text { ft. }\end{array}$ \\
\hline Wgi- & $\begin{array}{l}\text { Lyons, Harris } \\
\text { and } 0^{\prime} \text { Guinn }\end{array}$ & Harris No.l & $\begin{array}{l}\text { Sec.29, T295, RI3E, } \\
\text { Tampa(Beach Park) }\end{array}$ & $10 \pm$ & 1930 & .867 & $\begin{array}{l}\text { Bottom samples, } \\
\text { Eocene. Some gener- } \\
\text { al information. }\end{array}$ \\
\hline & $\begin{array}{l}\text { Southern } \mathrm{Pe-} \\
\text { troleum Co. }\end{array}$ & $\begin{array}{l}\text { Mizell \& Wil- } \\
\text { liams No. } 1\end{array}$ & $\begin{array}{l}\text { Jec. 4, T325i, R21E, } \\
\text { imauma, } 5 \mathrm{mi} \text {. NE }\end{array}$ & 120 & 1935 & 2,855 & $\begin{array}{l}\text { No samples or satis- } \\
\text { factory data. Junked } \\
\text { and abandoned war. } \\
15,1936 . \text { Jkidded over } \\
\text { to location No. } 2 \text {. }\end{array}$ \\
\hline & $\begin{array}{l}\text { Southern } \mathrm{Pe}- \\
\text { troleum Co. }\end{array}$ & $\begin{array}{l}\text { Mizell \& } \text { iill- } \\
\text { liams No. } 2\end{array}$ & $\begin{array}{l}\mathrm{Sec} .4, \mathrm{~T} 32 \mathrm{~S}, \mathrm{R} 2 \mathrm{IE}, \\
\text { Timauma, } 5 \mathrm{mi} \text {. NE }\end{array}$ & & 1936 & 964 & $\begin{array}{l}\text { Abandoned Nov.15, } \\
1936 . \text { No satis- } \\
\text { factory data. }\end{array}$ \\
\hline
\end{tabular}


HOLMES COUNTY

\begin{tabular}{|c|c|c|c|c|c|c|c|}
\hline $\begin{array}{l}\text { EGS } \\
\text { NO. }\end{array}$ & COMLANY Or OWNERS & $\begin{array}{l}\text { FARM or } \\
\text { WELL NAME }\end{array}$ & LOCATION & $\begin{array}{l}\text { ELEV- } \\
\text { ATION }\end{array}$ & \begin{tabular}{|l|} 
YEAR \\
COM- \\
PLETED \\
\end{tabular} & DEPTH & RELARKS \\
\hline 1513 & S. B. Breeding & $\begin{array}{l}\text { N.E. Coats } \\
\text { No. } I\end{array}$ & $\begin{array}{l}\text { sec.25, T7N, RI5 } \\
\text { Esto, } 4 \mathrm{mi} \text {. iN }\end{array}$ & $\begin{array}{l}195 \pm \mathrm{Grd} \\
202 \mathrm{DF}\end{array}$ & 1947 & 4,107 & $\begin{array}{l}375 \text { cuttings from } 0 \\
\text { to } 4107 \text { ft. Cores } \\
\text { from No. } 1 \text { @ } 2963- \\
2980 \text { to core No. } 4 \text { @ } \\
3650-3670 . \text { Schlum- } \\
\text { berger from } 11 \text { to } \\
4107 . \text { Driller's log } \\
\text { from 0 to } 4107 .\end{array}$ \\
\hline
\end{tabular}

JACKSON COUNTY

\begin{tabular}{|c|c|c|c|c|c|c|c|}
\hline${ }^{285}$ & $\begin{array}{l}\text { Mamie Hammonds, Mirs. } \\
\text { et al }\end{array}$ & $\begin{array}{l}\text { Granberry } \\
\text { No. } 1\end{array}$ & $\begin{array}{l}\text { Mec. } 15, T 5 N, \pi 9 \%, \\
\text { Marianna, } 8 \mathrm{mi} \\
\text { NE }\end{array}$ & $124 \mathrm{DF}$ & 1936 & 5,015 & $\begin{array}{l}249 \text { cuttings from } 200 \\
\text { to } 5015 \text { ft. Core } 3 \\
200-220 . \text { Also. cores } \\
\text { from No. } 13 \text { 1678 to } \\
\text { core No. } 40 \text { \$ } 5012- \\
5015 \text { with several } \\
16 \text { missing.Drillers } \\
\text { log from 0 to } 5015 \text {. }\end{array}$ \\
\hline
\end{tabular}




\begin{tabular}{|c|c|c|c|c|c|c|c|}
\hline $\begin{array}{l}\text { FGS } \\
\text { No. }\end{array}$ & COMPANY Or ONNERS & $\begin{array}{l}\text { FAPM Or } \\
\text { WELL NAME }\end{array}$ & LOCATION & $\begin{array}{l}\text { ELEV- } \\
\text { ATION }\end{array}$ & $\begin{array}{l}\text { YEAR } \\
\text { COM- } \\
\text { PLETED } \\
\end{array}$ & DEPTH & RENARKS \\
\hline$N-19$ & $\begin{array}{l}\text { Southern states } \\
\text { oil Corporation }\end{array}$ & $\begin{array}{l}\text { Niller \& Gossard } \\
\text { No. I }\end{array}$ & $\begin{array}{l}\text { Sec. } 17, \mathrm{~T} 2 \mathrm{~N}, \mathrm{R} 5 \mathrm{E} \\
\text { Monticello, } 2 \\
\mathrm{mi} \text {. NE }\end{array}$ & $230 \pm$ & 1928 & 3,838 & $\begin{array}{l}203 \text { cuttings from } 0 \\
\text { to } 3838 \text { ft.Cores } \\
1640,3051,3066 \text {. } \\
\text { Drilier s } 10 \text { from } \\
40 \text { to } 1633 \text {. }\end{array}$ \\
\hline
\end{tabular}

LAFAYETTE COUNTY

\begin{tabular}{|c|c|c|c|c|c|c|c|}
\hline 968 & sun 011 Company & 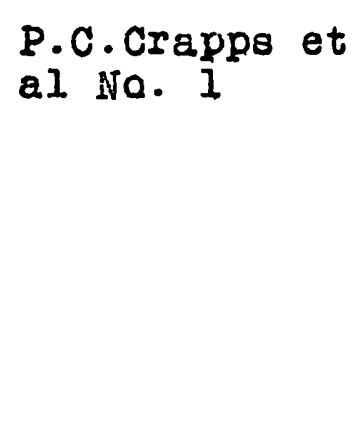 & $\begin{array}{l}\mathrm{sec} .25, \mathrm{~T} 6 \mathrm{~s}, \mathrm{Rl} \\
\text { ilayo, } 12 \mathrm{mi} . \mathrm{sE}\end{array}$ & $\begin{array}{l}65 \mathrm{Grd} \\
70 \mathrm{DF}\end{array}$ & 1945 & 4,133 & $\begin{array}{l}337 \text { cuttings from } 69 \\
\text { to } 4130 \text { ft.Cores } \\
\text { from No. } 1 \text {. } 1659- \\
1568 \text { to core No. } 180 \\
\text { } 4129-4133 . \text { Schlum- } \\
\text { berger froin } 70 \text { to } \\
4127 . \text { Driller s log } \\
\text { from 0 to } 4133 \text {. Core } \\
\text { record, other data. }\end{array}$ \\
\hline & $\begin{array}{l}\text { Humble oil \& Re- } \\
\text { fining Co. }\end{array}$ & $\begin{array}{l}\text { R.I. Henderson } \\
\text { Trustee et ux } \\
\text { No. } 1\end{array}$ & $\begin{array}{l}\text { sec. } 20, \mathrm{~T} 4 \mathrm{~s}, \mathrm{R} 1 \mathrm{IE} \\
\text { Nayo, } 6 \frac{1}{2} \mathrm{mi} . \mathrm{NiT}\end{array}$ & $52 \mathrm{DF}$ & & 3,357 & $\begin{array}{l}\text { December } 31,1947 \\
\text { shut down, temporari- } \\
\text { ly, at } 3217 \text { ft. on } \\
\text { account of exceed- } \\
\text { ingly high water. } \\
\text { Started again Jan.9, } \\
\text { l948. }\end{array}$ \\
\hline
\end{tabular}


LAKE COUNTY

\begin{tabular}{|c|c|c|c|c|c|c|c|}
\hline $\begin{array}{l}\text { FGS } \\
\text { No. }\end{array}$ & COMPANY Or OWNERS' & $\begin{array}{l}\text { FARM or } \\
\text { NELL NGME }\end{array}$ & LOCATION & $\begin{array}{l}\text { ELEV- } \\
\text { ATION }\end{array}$ & \begin{tabular}{|l|} 
YEAR \\
CONi- \\
PLETED \\
\end{tabular} & DEPTH & REMARKS \\
\hline 275 & $\begin{array}{l}\text { Oil Development } \\
\text { Company of Florida } \\
\\
\text { Union Oil \& Re- } \\
\text { fining Company }\end{array}$ & $\begin{array}{l}\text { South Lake } \\
\text { No. } 2\end{array}$ & $\begin{array}{l}\text { Sec. 17, T24s, R25E, } \\
\text { Groveland, } 12 \mathrm{mi} \text {. } \\
\text { s. } \\
\\
\text { sec.19, T24s, R25E, } \\
\text { Groveland, i2 } \mathrm{mi} . \\
\text { s. }\end{array}$ & 120 & 1936 & 255 & $\begin{array}{l}498 \text { cuttings from } 0 \\
\text { to } 3933 \text { ft., and } \\
5400-5500,5500-5600 \\
\text { ft. } 50 \text { cuttings from } \\
16 \text { cores. Samples and } \\
\text { cores preserved to } \\
\text { total depth, but not } \\
\text { in jurvey offices. } \\
\text { 80hlumberger from } \\
2200 \text { to } 6113 \text {. Drill- } \\
\text { ers log from o to } \\
\text { 1947. } \\
\text { No samples or other } \\
\text { satisfactory inform- } \\
\text { ation. Affiliated } \\
\text { with oil Development } \\
\text { Company of Florida. }\end{array}$ \\
\hline
\end{tabular}

LEE COUNTY

\begin{tabular}{|c|c|c|c|c|c|c|c|}
\hline $\begin{array}{l}\text { igi- } \\
112\end{array}$ & $\begin{array}{l}\text { Florida Exploration } \\
\text { Syndicate }\end{array}$ & $\begin{array}{l}\text { Consolidated } \\
\text { Land Co.No.1 }\end{array}$ & $\left|\begin{array}{l}\text { Sec. } 10, T 453, R 26 E, \\
\text { Ft.Myer } 8,12 \mathrm{mi} . \mathrm{SE}\end{array}\right|$ & 27 & 1923 & 2,191 & $\begin{array}{l}\text { Driller's log from } 0 \\
\text { to } 2187 \text { ft. No samp- } \\
\text { les. }\end{array}$ \\
\hline igi- & $\begin{array}{l}\text { Florida Exploration } \\
\text { Syndicate }\end{array}$ & $\begin{array}{l}\text { Consolidated } \\
\text { Land Co.NO.e }\end{array}$ & $\left|\begin{array}{l}\text { sec. } 21, T 445, \text { R26E } \\
\text { Ft.Myers, } 10 \text { mi. E }\end{array}\right|$ & & $1924 ?$ & 3,018 & $\begin{array}{l}\text { Driller's log from } 0 \\
\text { to } 3018 \text { No.sanples }\end{array}$ \\
\hline
\end{tabular}




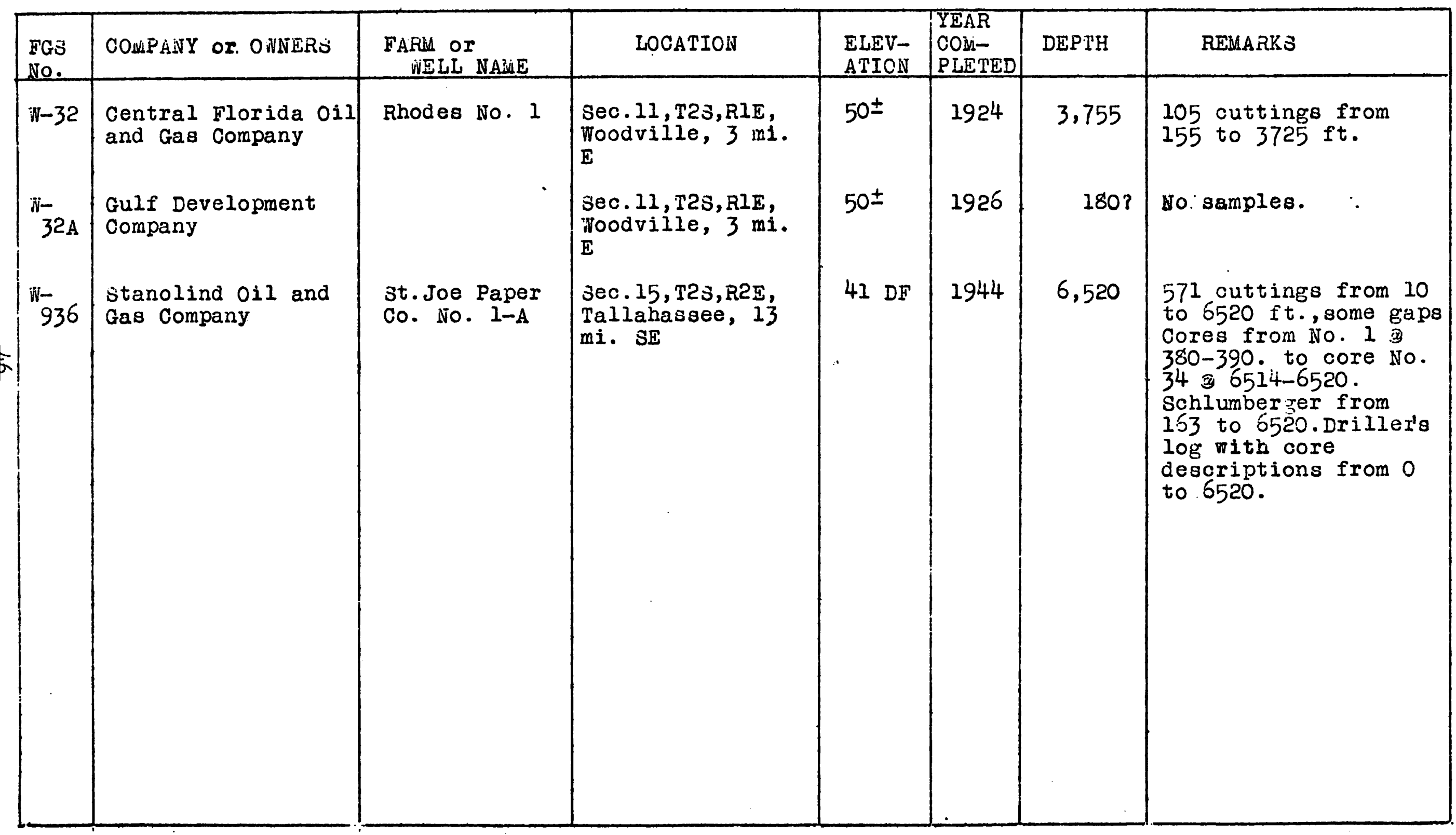




\begin{tabular}{|c|c|c|c|c|c|c|c|}
\hline $\begin{array}{l}\text { FGS } \\
\text { No. }\end{array}$ & CONLANY Or OWNERS & $\begin{array}{l}\text { FARM or } \\
\text { WELL NAME }\end{array}$ & LOCATION & $\begin{array}{l}\text { ELEV- } \\
\text { ATION }\end{array}$ & \begin{tabular}{|l|} 
YEAR \\
COUii- \\
PLETED \\
\end{tabular} & DEPTH & REMARKS̈ \\
\hline ivgi- & $\begin{array}{l}\text { Bronson inanufact- } \\
\text { uring Company }\end{array}$ & & $\begin{array}{l}\text { Sec.18, T12S, R17E, } \\
\text { Bronson }\end{array}$ & $70 \pm$ & 1927 & 717 & $\begin{array}{l}\text { No samples.Driller's } \\
\text { log from } 209 \text { to } 717 \\
\text { ft. }\end{array}$ \\
\hline & C.A. ialker et al & Walker No.1 & $\begin{array}{l}\sec .9, \mathrm{Tl} 15 \mathrm{~S}, \mathrm{R} I 3 \mathrm{E}, \\
\text { Cedar Keys, } 4 \mathrm{mi} . \\
\mathrm{NE}\end{array}$ & & 1928 & $400 \pm$ & $\begin{array}{l}\text { No definite informa- } \\
\text { tion or samples. Mrr. } \\
\text { vialker was a driller } \\
\text { on } 166 \text {. }\end{array}$ \\
\hline i-166 & $\begin{array}{l}\text { Suwannee Petroleum } \\
\text { Corporation }\end{array}$ & Sholtz No.l & $\begin{array}{l}\text { Sec.9, T15S, RI3E, } \\
\text { Cedar Keys, } 4 \mathrm{mi} . \\
\text { NE }\end{array}$ & 18 & 1929 & 4,010 & $\begin{array}{l}\text { Depth of } 4010 \text { reach- } \\
\text { ed June } 12,1929 \text {, al- } \\
\text { though tests were } \\
\text { made as late as June } \\
1,1932 \text {. Gas showing } \\
\text { recorded at } 4010 \text { ft. } \\
436 \text { cuttings from } \\
\text { 115 to } 4010 \text { ft. Drill- } \\
\text { er's log from o to } \\
4010 \text {. Water analysis. }\end{array}$ \\
\hline$i-355$ & $\begin{array}{l}\text { Florida } 011 \text { Dis- } \\
\text { covery Company }\end{array}$ & $\begin{array}{l}\text { Sholtz Land } \\
\text { No. } 2 \text { (No.1 } \\
\text { junked) }\end{array}$ & $\begin{array}{l}\text { Sec.9, T15s, Rl3E, } \\
\text { Cedar Keys, } 4 \mathrm{mi} . \\
\mathrm{NE}(1600 \mathrm{~N} \text { of } \\
\text { ii-165) }\end{array}$ & 9 & 1939 & 5,266 & $\begin{array}{l}263 \text { cuttings from } 40 \\
\text { to } 5256 \mathrm{ft} \text {. } 8 \text { cores } \\
\text { from } 4059-4062 \text { to } \\
4954-4965 \text {. Halibur- } \\
\text { ton } 3986 \text { to } 4948 \text {. } \\
\text { Driller's log from o } \\
\text { to } 5266 \text {. }\end{array}$ \\
\hline$. i-511$ & $\begin{array}{l}\text { C. } \text { i. Houlk \& D.A. } \\
\text { Harkey }\end{array}$ & $\begin{array}{l}\text { No. } 1 \text { Crapps } \\
\text { et al }\end{array}$ & $\begin{array}{l}\text { Sec. } 15, \mathrm{Tl} 4 \mathrm{~s}, \mathrm{Rl} 7 \mathrm{E}, \\
\text { Bronson, } 13 \frac{1}{2} \mathrm{mi} . \\
\mathrm{s}\end{array}$ & $\begin{array}{l}55 \\
35 \text { (USE } \\
\text { map) }\end{array}$ & 1940 & 1,310 & $\begin{array}{l}112 \text { cuttings from } 98 \\
\text { to } 1291 \text { ft.Driller's } \\
\text { log from } 1 \text { to } 1288 .\end{array}$ \\
\hline
\end{tabular}




\begin{tabular}{|c|c|c|c|c|c|c|c|}
\hline $\begin{array}{l}\text { FGS } \\
\text { No. }\end{array}$ & COLPANY or OUNERS & $\begin{array}{l}\text { FARNi or } \\
\text { WELL NAME }\end{array}$ & LOCATION & $\begin{array}{l}\text { ELEV- } \\
\text { ATION }\end{array}$ & \begin{tabular}{|l|} 
YEAR \\
COUL- \\
PLETED \\
\end{tabular} & DEPTH & REiliARKS \\
\hline 871 & $\begin{array}{l}\text { Frank A. Thompson, } \\
\text { Trustee, Thompson \& } \\
\text { Pollard Associates }\end{array}$ & $\begin{array}{l}\text { Levy-Lennon } \\
\text { Syndicate Inc. } \\
\text { Wo. I }\end{array}$ & $\begin{array}{l}\text { sec.16, Tl3s, Rl6E, } \\
\text { Bronson, } 8 \mathrm{mi} \text {. Si }\end{array}$ & $35 \mathrm{Grd}$ & 1944 & 679 & $\begin{array}{l}116 \text { samples from } 0 \text { to } \\
569 \text { ft. strong water } \\
\text { flow from about } 500 \\
\text { ft., also analysis of } \\
\text { water from that depth } \\
\text { Driller's log from } 0 \\
\text { to } 678 .\end{array}$ \\
\hline & $\begin{array}{l}\text { Frank A.Thompson, } \\
\text { Trustee, Thompson, F. } \\
\text { A.\&. ViacGregor, J.A. }\end{array}$ & $\begin{array}{l}\text { Levy-Lennon } \\
\text { Syndicate Inc. } \\
\text { No. } 2\end{array}$ & $\begin{array}{l}500^{\prime} \pi \text { \& } 12501 \mathrm{~N}, \mathrm{SE} \\
\text { cor.3ec.16, T13S, } \\
\text { Rl6E, Bronson, } 8 \\
\text { m1. S7 }\end{array}$ & & 1946 & 810 & $\begin{array}{l}\text { Drilled by Libby \& } \\
\text { Freeman, Drilling } \\
\text { Contractors, orlando }\end{array}$ \\
\hline$\frac{17}{1449}$ & $\begin{array}{l}\text { Frank A. Thompson, } \\
\text { Trustee }\end{array}$ & $\begin{array}{l}\text { Levy-Lennon } \\
\text { syndicate } \\
\text { No. } 3\end{array}$ & $\begin{array}{l}\text { Sec.15, Tl3s, Rl6:, } \\
\text { Bronson, } 8 \mathrm{mi} \text {. Sil }\end{array}$ & $35 \mathrm{Grd}$ & 1945 & 380 & $\begin{array}{l}41 \text { samples from } 0 \text { to } \\
380 \mathrm{ft} \text {. }\end{array}$ \\
\hline ii- & Sun 011 Company & $\begin{array}{l}\text { J.T. Goe the } \\
\text { NO. I }\end{array}$ & $\begin{array}{l}\text { Sec. } 31, \mathrm{TI} 4 \mathrm{~S}, \mathrm{R} 17 \mathrm{E}, \\
\text { Gulf Hammock, } 4 \\
\text { mi. SE }\end{array}$ & $\begin{array}{l}29 \mathrm{Grd} \\
34 \mathrm{DF}\end{array}$ & 1946 & 3,997 & 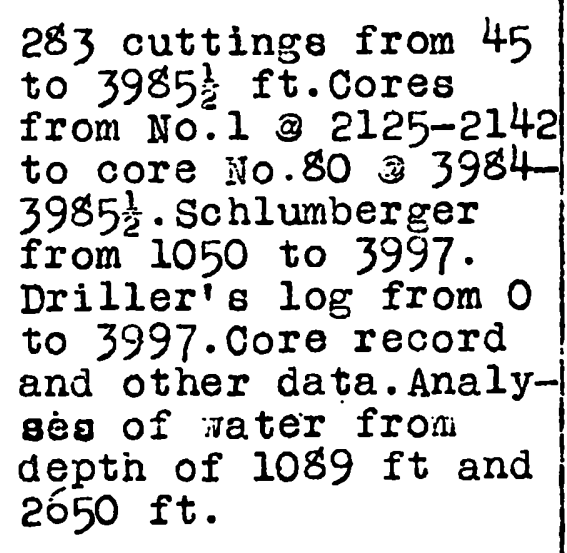 \\
\hline $\begin{array}{l}i v- \\
1537\end{array}$ & $\begin{array}{l}\text { Coastal Petroleum } \\
\text { Company }\end{array}$ & $\begin{array}{l}\text { James B. \& } \\
\text { Julian P. Rag- } \\
\text { land No. } 1\end{array}$ & 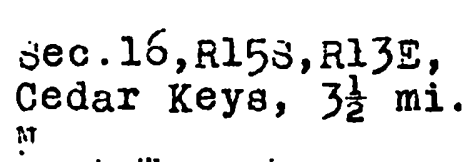 & $\begin{array}{l}5 \text { Grd } \\
15 \text { DF } \\
\text { Re?ly }\end{array}$ & $\begin{array}{l}1947 \\
\text { nahind }\end{array}$ & 5,850 & $\begin{array}{l}14 \text { cuttings from } 10 \\
110 \text { ft. No cuttings } \\
\text { from i10 to } 380 \text { ft. }\end{array}$ \\
\hline
\end{tabular}


LEVY COUNTY - Continued

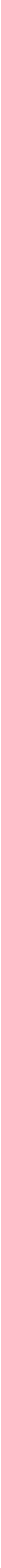




\section{LIBERTY COUNTY}

\begin{tabular}{|c|c|c|c|c|c|c|c|}
\hline $\begin{array}{l}\text { FGS } \\
\text { No. }\end{array}$ & CONiPANY OT OWINERO & $\begin{array}{l}\text { FARM or } \\
\text { WELL NAWHE }\end{array}$ & LOCATION & $\begin{array}{l}\text { ELEV- } \\
\text { ATION }\end{array}$ & $\begin{array}{l}\text { YEAR } \\
\text { COW- } \\
\text { PLETED } \\
\end{array}$ & DEPTH & REinARKS \\
\hline $\begin{array}{l}\pi- \\
1106\end{array}$ & Pure oil Company & $\begin{array}{l}\text { Neal Lumber \& } \\
\text { Manufacturing } \\
\text { Co., No. } 1\end{array}$ & $\begin{array}{l}\sec .33, \mathrm{~T} 2 \mathrm{~s}, \mathrm{R} 8 \mathrm{ir} \\
\text { orange, } 3 \mathrm{mi} \text {. N }\end{array}$ & 55Grd & 1946 & 4,506 & $\begin{array}{l}\text { l20 cuttings from } 12 \\
\text { to } 4496 \text { ft. schlum- } \\
\text { berger from } 861 \text { to } \\
4502 . \text { Driller' } 8 \text { log } \\
\text { from } 0 \text { to } 4506\end{array}$ \\
\hline 1385 & Pure Oil Company & $\begin{array}{l}\text { Gex \& Lewin } \\
\text { No. I }\end{array}$ & $\begin{array}{l}\text { sec.24, T53,R6 } \\
\text { sumstra, } 12 \mathrm{mi} . \mathrm{E}\end{array}$ & 25Grd & 1946 & 4,744 & $\begin{array}{l}152 \text { cuttings from } 0 \\
\text { to } 4744 \text { ft. schlum- } \\
\text { berger from } 942 \text { to } \\
4745 \text {. Driller's log } \\
\text { from o to } 4744 \text {. }\end{array}$ \\
\hline
\end{tabular}

IMADIJON COUNTY

\begin{tabular}{|c|c|c|c|c|c|c|c|}
\hline & Hunt Oil Company & $\begin{array}{l}\text { J.N.Gibson } \\
\text { No. } 1\end{array}$ & $\mid \begin{array}{l}\text { Sec.16, Tls, RlIE, } \\
\text { Ellavilie, } 4 \mathrm{mi} . \mathrm{t}\end{array}$ & $\begin{array}{l}86 \mathrm{Grd} \\
91 \mathrm{DF}\end{array}$ & 1943 & 3,380 & $\begin{array}{l}\text { No samples. Schlum- } \\
\text { berger from } 301 \text { to } \\
3333 \text {. }\end{array}$ \\
\hline 1596 & Hunt Oil Company & $\begin{array}{l}\text { J.ir.Gibson } \\
\text { No. } 2\end{array}$ & $\begin{array}{l}\text { Sec.6, TlS, RIOE, } \\
\text { Madison, } 4 \mathrm{ml} \text {. SE }\end{array}$ & $\begin{array}{l}102 \mathrm{Grd} \\
107 \mathrm{DF}\end{array}$ & 1944 & 5,381 & $\begin{array}{l}422 \text { cuttings from } \\
105 \text { to } 5381 \text { ft. } 3 \\
\text { cores. Schlumber- } \\
\text { ger fram } 225 \text { to } 5385\end{array}$ \\
\hline 1597 & Hunt Oil Company & $\begin{array}{l}\text { J. } \dddot{G} \text { Gibson } \\
\text { No. } 3\end{array}$ & $\begin{array}{l}\text { Sec. } 18, T 2 S, R 11 \mathrm{~T}, \\
\text { Lee, } 3 \mathrm{mi} . \mathrm{SE}\end{array}$ & $\begin{array}{l}84 \mathrm{Grd} \\
89 \mathrm{DF}\end{array}$ & 1944 & 3,667 & $\begin{array}{l}256 \text { cuttings from } \\
320 \text { to } 3550 \text { ft. } 7 \\
\text { cores } 559-579 \text { to } \\
3526-3540.3 c h l \text { mber- } \\
\text { ger from } 310 \text { to } 3540\end{array}$ \\
\hline
\end{tabular}


LIADIJON COUNTY - Continued

\begin{tabular}{|c|c|c|c|c|c|c|c|}
\hline $\begin{array}{l}\text { FGS } \\
\text { NO. }\end{array}$ & COMPANY Or O ONERS & $\begin{array}{l}\text { FARiin or } \\
\text { NELL NALEE }\end{array}$ & LOCATION & $\begin{array}{l}\text { ELEV- } \\
\text { ATION }\end{array}$ & \begin{tabular}{|l|} 
YEAR \\
COM- \\
PLETED \\
\end{tabular} & DEPTH & REMARKS \\
\hline 1598 & Hunt Oil Company & $\begin{array}{l}\text { J. iv. Gibson } \\
\text { No. } 4\end{array}$ & $\begin{array}{l}\sec \cdot 5, \mathrm{~T} 2 \mathrm{~S}, \mathrm{RIIE} \\
\text { Lee, } 6 \mathrm{mi} \text {. SE }\end{array}$ & $\begin{array}{l}68 \mathrm{Grd} \\
73 \mathrm{DF}\end{array}$ & 1945 & 4,069 & $\begin{array}{l}227 \text { cuttings from } 900 \\
\text { to } 3750 \text { ft. } 3 \text { coreg } \\
\text { Schlumberger from } 866 \\
\text { to } 4078\end{array}$ \\
\hline
\end{tabular}

\section{MANATEE COUNTY}

\begin{tabular}{|c|c|c|c|c|c|c|c|}
\hline$\pi-23$ & $\begin{array}{l}\text { Tri-County oil } \\
\text { Company }\end{array}$ & $\begin{array}{l}\text { iN.E.Robinson } \\
\text { No. } 1\end{array}$ & $\begin{array}{l}\sec .15, \mathrm{~T} 34 \mathrm{~s}, \mathrm{RI} 7 \mathrm{E}, \\
\mathrm{Palmetto}, 1 \frac{1}{4} \mathrm{mi} . \\
\mathrm{N} .\end{array}$ & $9 \pm$ & 1925 & 1,265 & $\begin{array}{l}84 \text { samplea from } 70 \\
\text { to } 1025 \text { ft. }\end{array}$ \\
\hline $\begin{array}{l}\text { iigi- } \\
211\end{array}$ & $\begin{array}{l}\text { Tri-County } 0 i 1 \\
\text { Company }\end{array}$ & $\begin{array}{l}\text { H. E.Robinson } \\
\text { No. } 2\end{array}$ & $\begin{array}{l}\text { sec. } 15, \mathrm{~T} 345, \mathrm{Rl} 7 \mathrm{E} \\
\text { Palmetto, } 1 \frac{1}{4} \mathrm{mi} \\
\mathrm{N} i\end{array}$ & $9 \pm$ & 1927 & 2,665 & $\begin{array}{l}\text { Driller's log from } 0 \\
\text { to } 2660 \mathrm{ft} \text {. }\end{array}$ \\
\hline "108 & Surprise Oil Co. & $\begin{array}{l}\text { F.J. Mack } \\
\text { No. } 1\end{array}$ & $\begin{array}{l}\text { Sec. } 15 ?, \mathrm{~T} 34 \mathrm{~s}, \mathrm{R} 17 \mathrm{E} \\
\text { Palmetto, } 1 \mathrm{mi} . \\
\mathrm{Nii}\end{array}$ & $9 \pm$ & 1932 & $792 \pm$ & $\begin{array}{l}\text { No very satisfactory } \\
\text { data. }\end{array}$ \\
\hline
\end{tabular}




\section{NARION COUNTY}

\begin{tabular}{|c|c|c|c|c|c|c|c|}
\hline $\begin{array}{l}\text { FGS } \\
\text { No. }\end{array}$ & COMLPANY or OUNERS & $\begin{array}{l}\text { FARMi or } \\
\text { WELL NAWE }\end{array}$ & LOCATION & $\begin{array}{l}\text { ELEV- } \\
\text { ATION }\end{array}$ & $\begin{array}{l}\text { YEAR } \\
\text { COM- } \\
\text { PLITED } \\
\end{array}$ & DEPTH & REMARKS \\
\hline-18 & $\begin{array}{l}\text { Ocala oil Corpor- } \\
\text { ation }\end{array}$ & York No. 1 & $\begin{array}{l}\text { Sec. } 10, \text { T16s, R2OE } \\
\text { York, } 4 \text { mi. s }\end{array}$ & $90 \mathrm{Grd}$ & 1928 & 6,180 & $\begin{array}{l}152 \text { samples from } 0 \text { to } \\
60 \text { e ft. Driller's } \\
\text { log from 0 to } 5980 .\end{array}$ \\
\hline$x-77$ & $\begin{array}{l}\text { Flesher Petroleum } \\
\text { Corporation }\end{array}$ & & $\begin{array}{l}\text { Sec. } 35, \mathrm{Tl} 4 \mathrm{~s}, \mathrm{R} 2 \mathrm{E} \\
\text { Feliowship, } 1 \mathrm{mi} \\
\text { E, on dicDavid Hil }\end{array}$ & $155=$ & 1928 & $1,020 ?$ & $\begin{array}{l}53 \text { samples from } 285 \\
\text { to } 920 \mathrm{ft} \text {. }\end{array}$ \\
\hline iv- & J.3. Cosden, Ino. & $\begin{array}{l}\text { i.L. Lawson } \\
\text { No. } 1\end{array}$ & $\begin{array}{l}\text { Sec. } 25, \mathrm{Pl} 33, \mathrm{RaOE} \\
\text { Fairfield, } 2 \mathrm{mi} \\
\text { s }\end{array}$ & 193Grd & 1928 & 4,334 & $\begin{array}{l}\text { lo samples from } 945 \\
\text { to } 4334 \text { ft. Driller's } \\
\text { log from } 0 \text { to } 4334 \text {. }\end{array}$ \\
\hline$\frac{i-4}{1482}$ & sun $0 i l$ Company & $\begin{array}{l}\text { Henry in.Camp } \\
\text { No. } 1\end{array}$ & $\begin{array}{l}\text { Sec.16, T16s, R23E } \\
\text { Candler, } 3 \mathrm{mi} \text {. Ni }\end{array}$ & $\begin{array}{l}64 \mathrm{Grd} \\
74 \mathrm{DF}\end{array}$ & 1947 & 4,637 & $\begin{array}{l}9 \text { cuttings from } 35 \text { to } \\
153 \text { ft. No cuttings } \\
\text { from } 153 \text { to } 2000 \text { ft. } \\
278 \text { cuttings from } \\
2000 \text { to } 4637 \text { ft } 23 \\
\text { cores from No. i } 8 \\
1838-1848 \text { to core No. } \\
23 @ 4618-4623 \frac{1}{2} \text {. } \\
\text { Schlumberger from } 91 \\
\text { to } 4635 \text {. Driller's } \\
\text { log from o to } 4623 \frac{1}{2} \text {. } \\
\text { Water analysis. }\end{array}$ \\
\hline & & & & & & & \\
\hline
\end{tabular}


MONROE COUNTY

\begin{tabular}{|c|c|c|c|c|c|c|c|}
\hline $\begin{array}{l}\text { FGS } \\
\text { NO. }\end{array}$ & CONPANY Or OUINERS & $\begin{array}{l}\text { FARM Or } \\
\text { OUELL NAME }\end{array}$ & LOCATION & $\begin{array}{l}\text { ELEV- } \\
\text { ATION }\end{array}$ & $\begin{array}{l}\text { YEAR } \\
\text { COM- } \\
\text { PLETEI } \\
\end{array}$ & DEPTH & REMARKS \\
\hline $4 \sqrt{4}-$ & $\begin{array}{l}\text { Peninsular oil \& } \\
\text { Refining Gompany }\end{array}$ & 更 & $\begin{array}{l}\text { Sec. } 6, \text { T55s, R34 } \\
\text { Pinecrest }\end{array}$ & $14 D F$ & 1939 & 10,006 & $\begin{array}{l}664 \text { samples from } 14 \\
\text { to } 10,006 \text { ft. } 30 \\
\text { cores taken inter- } \\
\text { mittently from } 3252- \\
8395 \text { and } 801 \text { idly } \\
\text { from } 8395-10,006 \text {. } \\
\text { First well in Flori- } \\
\text { da to drill to more } \\
\text { than } 10,000 \text { ft. } \\
\text { Driller's log from } \\
0 \text { to } 10,006 \text {. }\end{array}$ \\
\hline 902 & $\begin{array}{l}\text { Republic oif } \\
\text { Company }\end{array}$ & $\begin{array}{l}\text { O. D. Robinson } \\
\text { state No. } 1 \text {, } \\
\text { Blanchard } \\
\text { concession }\end{array}$ & $\begin{array}{l}\text { Sec. } 29, \text { T59s, R40E } \\
\text { On upper Key } \\
\text { Largo in Barnes } \\
\text { sound }\end{array}$ & $23 \mathrm{DF}$ & 1946 & 12,051 & $\begin{array}{l}595 \text { samples from } 90 \\
\text { to } 11,878 \text { ft. None } \\
\text { from } 0 \text { to } 90 \text { ft. or } \\
\text { from } 170 \text { to } 520 \text { ft. } \\
\text { Cores from No. } 1 \text { 3 } \\
1468-1478 \text { to core No } \\
112312,050 \text {. } 3 \text { chlum } \\
\text { berger from } 6198 \text { to } \\
12,053 \text { Driller's log } \\
\text { from to } 12,051, \\
\text { with core descript- } \\
\text { ions. }\end{array}$ \\
\hline 1413 & $\begin{array}{l}\text { Gulf oil } \\
\text { Corporation }\end{array}$ & $\begin{array}{l}\text { State of } \\
\text { Florida NO.1 } \\
\text { Lease No.374 }\end{array}$ & $\begin{array}{l}\text { sec. } 15, \text { T } 67 \mathrm{~S}, \mathrm{R} 27 \mathrm{E} \\
\text { sugar Loaf Key } \\
\text { area, 16 mi. } \\
\text { eastivard from } \\
\text { Key vest }\end{array}$ & $\begin{array}{ll}00 & \mathrm{Grd} \\
23 & \mathrm{DF}\end{array}$ & 1947 & 6,100 & $\begin{array}{l}83 \text { samples from } 180 \\
\text { to } 1220 \text { ft. Lost re- } \\
\text { turns. Cores from } \\
\text { No.1 @ } 4950-4970 \text { to } \\
\text { core No.11 } 3695- \\
6100 \text {. Schlumberger } \\
\text { from620 to 6086. Drill } \\
\text { ers log fromoto } 6100 \text {. }\end{array}$ \\
\hline
\end{tabular}


MONROE COUNTY - Continued

\begin{tabular}{|c|c|c|c|c|c|c|c|}
\hline $\begin{array}{l}\text { FGS } \\
\text { NO. }\end{array}$ & COInPANY Or OHNERS & $\begin{array}{l}\text { FARM Or } \\
\text { WELL NAME }\end{array}$ & LOCATION & $\begin{array}{l}\text { ELEV- } \\
\text { ATION }\end{array}$ & $\begin{array}{l}\text { YEAR } \\
\text { COL- } \\
\text { PLETED }\end{array}$ & DEPTH & REMARKS \\
\hline 972 & $\begin{array}{l}\text { Gulf oil Corpor- } \\
\text { ation }\end{array}$ & $\begin{array}{l}\text { State of Flor- } \\
\text { ida J.P. Scran- } \\
\text { No. } 1 \text { - Lease } \\
\text { No. } 373\end{array}$ & $\begin{array}{l}\text { Sec. } 2, \text { T675, R29E } \\
\text { Big Pine Key area } \\
30 \text { mi. eastward } \\
\text { from Key Nest. }\end{array}$ & $\begin{array}{l}00 \cdot \mathrm{Grd} \\
23 \mathrm{DF}\end{array}$ & 1947 & 15,455 & $\begin{array}{l}849 \text { cuttings from } \\
317 \text { to } 15,455 \text { ft. } \\
\text { Cores from No.2 } 3 \\
317-332 \text { to core No. } \\
175 \text { 3 } 15,449-15,455 . \\
3 c h 1 u m b e r g e r \text { from } \\
172 \text { to } 15,452 . \text { Drill } \\
\text { er's } 10 \mathrm{~g} \text { from } 24 \text { to } \\
15,455 \text { Deepest well } \\
\text { in Florida. }\end{array}$ \\
\hline
\end{tabular}

NASTAU COUNTY

\begin{tabular}{|c|c|c|c|c|c|c|c|}
\hline 336 & $\begin{array}{l}\text { St. Wary's River oil } \\
\text { Corporation }\end{array}$ & $\begin{array}{l}\text { Hilliard Tur- } \\
\text { pentine Co. } \\
\text { No. I }\end{array}$ & $\begin{array}{l}\sec 19, T 4 \mathrm{~N}, \mathrm{R} 24 \mathrm{E} \\
\text { Hililard, } 4 \mathrm{mi} \text {. } \\
\text { Ni }\end{array}$ & $\begin{array}{l}99 \mathrm{Grd} \\
110 \mathrm{DF}\end{array}$ & 1940 & 4,824 & $\begin{array}{l}678 \text { samples from } 10 \\
\text { to } 4822 \text { ft. Drill- } \\
\text { er's log from } 0 \text { to } \\
4824 \text {. }\end{array}$ \\
\hline
\end{tabular}


OKALOOSA COUNTY

\begin{tabular}{|c|c|c|c|c|c|c|c|}
\hline $\begin{array}{l}\text { FGS } \\
\text { NO. }\end{array}$ & COLPANY OI OUNERS & $\begin{array}{l}\text { FARM or } \\
\text { WELL NAME }\end{array}$ & LOCATION & $\begin{array}{l}\text { ELEV- } \\
\text { ATION }\end{array}$ & $\begin{array}{l}\text { YEAR } \\
\text { COL- } \\
\text { PLETED } \\
\end{array}$ & DEPTH & REPLARKS \\
\hline $\begin{array}{l}\mathrm{N}- \\
1536\end{array}$ & $\begin{array}{l}\text { R.T. Adams, Independ- } \\
\text { ent Operator }\end{array}$ & $\begin{array}{l}\text { B.H.Hart, JI. } \\
\text { No. } 1\end{array}$ & $\begin{array}{l}\mathrm{Sec} \cdot 5, \mathrm{~T} 4 \mathrm{~N}, \mathrm{R} 22 \mathrm{w} \\
\text { Oak Grove, } 5 \mathrm{mi} . \\
\mathrm{SE}\end{array}$ & $195 \mathrm{DF}$ & 1947 & 5,505 & $\begin{array}{l}587 \text { samples from } 640 \\
\text { to } 5507 \text { ft. One cor } \\
\text { No. } 1 \text { \& } 5023-5028 . \\
\text { Schlumberger. from } \\
\text { lig to } 5504 . \text { Drill- } \\
\text { er's log from } 0 \text { to } \\
5505 \text {. }\end{array}$ \\
\hline
\end{tabular}

ORANGE COUNTY

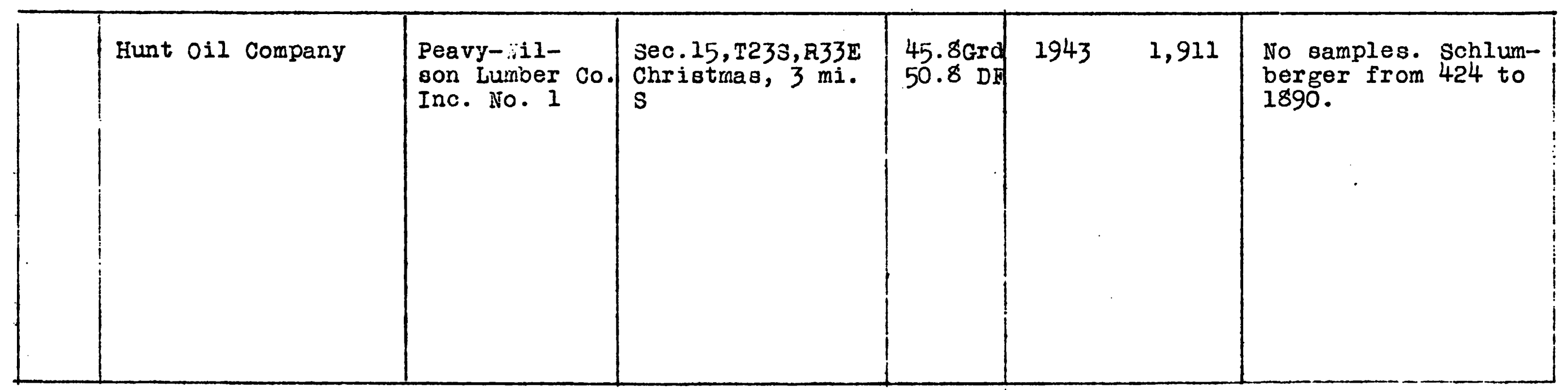


OSCEOLA COUNTY

\begin{tabular}{|c|c|c|c|c|c|c|c|}
\hline $\begin{array}{l}\text { FGS } \\
\text { NO. }\end{array}$ & COLiPANY or O.WNERS & $\begin{array}{l}\text { FARM or } \\
\text { WELL HAMUE }\end{array}$ & LOCATION & $\begin{array}{l}\text { ELEV- } \\
\text { ATIOIN }\end{array}$ & $\begin{array}{l}\text { YEAR } \\
\text { COMin- } \\
\text { PLETED } \\
\end{array}$ & DEPTH & RELARKS \\
\hline igi- & $\begin{array}{l}\text { Pennoylvania oil } \\
\text { Company }\end{array}$ & McCool Farm & $\begin{array}{l}\text { sec. 25, T26s, R29E } \\
\text { Kissimmee, } 8 \mathrm{mi} . \\
\text { S }\end{array}$ & & 1917 & 2,000 & $\begin{array}{l}\text { Descriptive los from } \\
0 \text { to } 2000 \text { ft., but } \\
\text { samples later lost } \\
\text { because of wet } \\
\text { storage. }\end{array}$ \\
\hline & Hunt Oil Company & $\begin{array}{l}\text { Consolidated } \\
\text { Naval Stores } \\
\text { No. } 1\end{array}$ & $\begin{array}{l}\text { Sec. 28, T26S, R32E } \\
\text { Tohopkee, } 3 \text { mi. SW }\end{array}$ & $\begin{array}{l}74 \mathrm{Grd} \\
79 \mathrm{DF}\end{array}$ & 1943 & 715 & $\begin{array}{l}\text { Abandoned because of } \\
\text { cavity trouble at } \\
\text { about } 700 \mathrm{ft} \text {. }\end{array}$ \\
\hline & Hunt Oil Company & $\begin{array}{l}\text { Consolidated } \\
\text { Naval stores } \\
\text { No. } 2\end{array}$ & $\begin{array}{l}\mathrm{Sec} .21, \mathrm{~T} 27 \mathrm{~s}, \mathrm{R} 33 \mathrm{E} \\
\text { Holopaw, } 4 \mathrm{mi} . \mathrm{SE}\end{array}$ & $\begin{array}{l}56 \mathrm{Grd} \\
61 \mathrm{DF}\end{array}$ & 1943 & 2,030 & $\begin{array}{l}\text { No samples. Schlum- } \\
\text { berger from } 558 \text { to } \\
\text { 1999. }\end{array}$ \\
\hline 1014 & $\begin{array}{l}\text { Humble oil \& Re- } \\
\text { fining Company }\end{array}$ & $\begin{array}{l}\text { N. Ray Car- } \\
\text { roll No. I }\end{array}$ & $\begin{array}{l}\text { Sec. 10, T275, R34E } \\
\text { Deer Park, } 4 \mathrm{mi} . \mathrm{N}\end{array}$ & $\begin{array}{l}46 \mathrm{Grd} \\
62 \mathrm{DF}\end{array}$ & 1946 & $8,044 \frac{1}{2}$ & 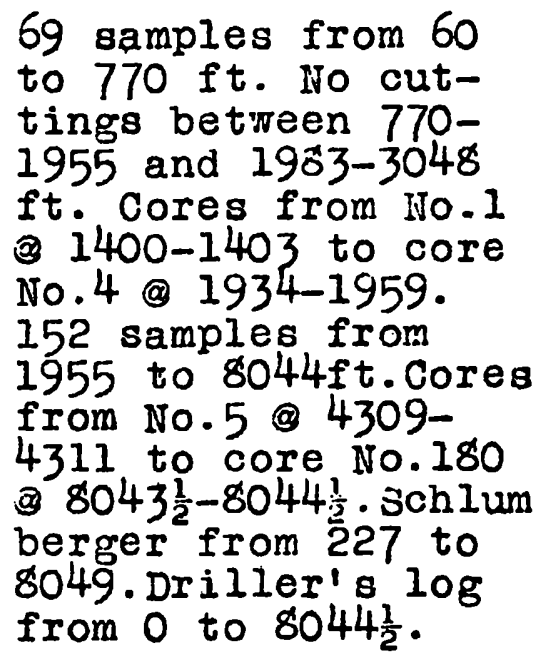 \\
\hline 1411 & $\begin{array}{l}\text { Humble oil \& Re- } \\
\text { fining Company }\end{array}$ & $\begin{array}{l}\text { i.P. Hayman, } \\
\text { et ux No. } 1\end{array}$ & $\begin{array}{l}\text { sec.12, T31s, R33E } \\
\text { Apoxsee, } 3 \mathrm{mi} \text {. }\end{array}$ & $\begin{array}{l}68 \mathrm{Grd} \\
88 \mathrm{DF}\end{array}$ & 1946 & 8,798 & $\begin{array}{l}328 \text { samples from } 261 \\
\text { to } 8780 \mathrm{ft} \text {. Cores }\end{array}$ \\
\hline
\end{tabular}


OSCEOLA COUNTY - Continued

\begin{tabular}{|c|c|c|c|c|c|c|c|}
\hline $\begin{array}{l}\text { FGS } \\
\text { No. }\end{array}$ & COUPANY Or OHNERS & $\begin{array}{l}\text { FARM or } \\
\text { NELE NASE }\end{array}$ & LOCATION & $\begin{array}{l}\text { ELEV- } \\
\text { AIION }\end{array}$ & $\begin{array}{l}\text { YEAR } \\
\text { CON- } \\
\text { PLETED }\end{array}$ & DEPTH & RE'WARKS \\
\hline 1411 & Continued & & - & & & & $\begin{array}{l}\text { from No.1 @ } 888-904 \\
\text { to core No.283 } \\
8797-8798 . \text { Schlum- } \\
\text { ber.er from } 332 \text { to } \\
8798 . \text { Driller's 10g } \\
\text { from 0 to } 8781 . \text { Also } \\
\text { core descriptions } \\
\text { from core No. } 1 \text { @ } \\
888-904 \text { to core No. } \\
283 \text { @ } 8797-8798 .\end{array}$ \\
\hline
\end{tabular}

PALUA BEACH COUNTY

\begin{tabular}{|c|c|c|c|c|c|c|c|}
\hline 1471 & $\begin{array}{l}\text { Humble oil \& Re- } \\
\text { fining Company }\end{array}$ & $\begin{array}{l}\text { Tucson Corp- } \\
\text { oration No.I } \\
\end{array}$ & $\begin{array}{l}\text { sec. } 35, \mathrm{~T} 43 \mathrm{~s}, \mathrm{R} 40 \mathrm{E} \\
\text { Loxaha tchee, } 3 \mathrm{mi} . \\
\text { ii }\end{array}$ & $\begin{array}{l}14 \mathrm{Grd} \\
34 \mathrm{DF}\end{array}$ & 1947 & 13,375 & $\begin{array}{l}\text { No samples from o } \\
\text { to } 510 \text { ft. } 110 \text { samp- } \\
\text { les from } 510 \text { to } \\
1530 \text { ft., none from } \\
1630 \text { to } 5564 \text { ft. } \\
371 \text { samples from } \\
5664 \text { to } 13,350 \text { ft. } \\
\text { Cores from No.1 } 3 \\
3115-3120 \text { to core } \\
\text { No.246 @ } 13,365- \\
13,375 . \text { schlurnberger } \\
\text { from } 149 \text { to } 13,371 \text {. } \\
\text { Driller's log from } \\
41 \text { to } 13,375 \text {. }\end{array}$ \\
\hline
\end{tabular}




\section{PASCO COUNTY}

\begin{tabular}{|c|c|c|c|c|c|c|c|}
\hline $\begin{array}{l}\text { FGs } \\
\text { No. }\end{array}$ & COMPAIVY Or O IrNERS & $\begin{array}{l}\text { Firiva or } \\
\text { WELL NAWE }\end{array}$ & LOCATION & $\begin{array}{l}\text { ELEV- } \\
\text { ATION }\end{array}$ & $\begin{array}{l}\text { YEAR } \\
\text { COW- } \\
\text { PLETEL }\end{array}$ & DEPTH & REîARKS \\
\hline & $\begin{array}{l}\text { J.H.Critchlow } \\
\text { P.E.Bobb }\end{array}$ & & $\begin{array}{l}\text { sec.32, T245, R22E } \\
\text { Dade City, } 4 \mathrm{mi} . \\
\text { sec.34, T25s, R21E } \\
\text { zephyrhilis, } 2 \mathrm{mi} . \\
N\end{array}$ & & $\begin{array}{l}1917 \\
1932\end{array}$ & $\begin{array}{l}2,200 \\
1,003\end{array}$ & $\begin{array}{l}\text { No samples or other } \\
\text { satisfactory data. } \\
\text { No samples or other } \\
\text { satisfactory data. }\end{array}$ \\
\hline
\end{tabular}

पn

\section{PINELLAS COUNTI}

\begin{tabular}{|c|c|c|c|c|c|c|c|}
\hline \multirow[t]{4}{*}{$i-60$} & $\begin{array}{l}\text { Pomello oil Co., } \\
\text { C. it.Houk, st. } \\
\text { Petersburg }\end{array}$ & & $\begin{array}{l}\text { Safety Harbor, } 2 \\
\text { mi. }\end{array}$ & $40 \pm$ & 1927 & 846 & $\begin{array}{l}66 \text { samples from } 15 \mathrm{tq} \\
836 \mathrm{ft} .\end{array}$ \\
\hline & $\begin{array}{l}\text { Intercoastal Devel- } \\
\text { opment Co. }\end{array}$ & & $\begin{array}{l}\text { Sec.10, T275, Rló } \\
\text { Tarpon springs, } \frac{1}{2} \\
\text { mi. I }\end{array}$ & $20 \pm$ & 1928 & 620 & $\begin{array}{l}\text { No samples or other } \\
\text { satisfactory data. }\end{array}$ \\
\hline & Nevers-Yerxa & $\begin{array}{l}\text { Nevers-Yerxa } \\
\text { No. } 1\end{array}$ & $\begin{array}{l}\text { sec. } 2, \mathrm{~T} 29 \mathrm{~s}, \mathrm{R} 15 \mathrm{E} \\
\text { Clearwater, } 2 \mathrm{mi} \text {. } \\
\mathrm{NE}\end{array}$ & $40 \pm$ & 1930 & 998 & $\begin{array}{l}\text { No samples or other } \\
\text { satisfactory data. }\end{array}$ \\
\hline & Yerxa-inalone & $\begin{array}{l}\text { Nevers-Yerxa } \\
\text { No. } 2\end{array}$ & clearwater & & 1930 & 995 & $\begin{array}{l}\text { No samples or other } \\
\text { satisfactory data. }\end{array}$ \\
\hline 221 & $\begin{array}{l}\text { Pioneer } 0 i 1 \text { \& } \\
\text { iining Co. }\end{array}$ & $\begin{array}{l}\text { Nevers-Yerxa } \\
\text { No. } 3\end{array}$ & $\begin{array}{l}\text { sec.2, T29s, Rl6E } \\
\text { clearwater }\end{array}$ & $35^{+}$ & 1932 & 1,534 & $\begin{array}{l}12 \text { cores from dif- } \\
\text { erent depths from } \\
866 \frac{1}{2} \text { to } 1534 \mathrm{ft} .\end{array}$ \\
\hline
\end{tabular}


PINELLAS COUNTY - Continued

\begin{tabular}{|c|c|c|c|c|c|c|c|}
\hline $\begin{array}{l}\text { FGS } \\
\text { NO. }\end{array}$ & CONAPAHY OY OWNERS & $\begin{array}{l}\text { FAFin or } \\
\text { NELI NAME }\end{array}$ & LOCATION & $\begin{array}{l}\text { ELEV- } \\
\text { ATION }\end{array}$ & $\begin{array}{l}\text { YEAR } \\
\text { COL- } \\
\text { PLETED }\end{array}$ & DEPTH & REMARKS \\
\hline & $\begin{array}{l}\text { Gulf Tidewater } 0 i l \\
\text { Company }\end{array}$ & \multirow{3}{*}{$\begin{array}{l}\text { Fugazzi } \\
\text { Property }\end{array}$} & $\begin{array}{l}\sec .4, \operatorname{Tag} 3, R I 6 E \\
\text { Palm Harbor, } 3 \\
\text { mi. E }\end{array}$ & \multirow{2}{*}{\multicolumn{2}{|c|}{1930}} & \multirow[b]{3}{*}{1,161} & $\begin{array}{l}\text { No samples or other } \\
\text { satisfactory data. }\end{array}$ \\
\hline & $\begin{array}{l}\text { Gillespie-Christian } \\
\text { et al }\end{array}$ & & clearwater & & & & $\begin{array}{l}\text { No samples or other } \\
\text { satiofactory data. }\end{array}$ \\
\hline & $\begin{array}{l}\text { Gulf Tidewater Pro- } \\
\text { duction Company }\end{array}$ & & $\begin{array}{l}\text { sec. } 11, \text { T32S, RI6E } \\
\text { st. Petersburg, } 4 \\
\text { mi. S }\end{array}$ & & 1937 & & $\begin{array}{l}\text { No samples or other } \\
\text { satisfactory data. }\end{array}$ \\
\hline & R.E.Skinner & $\begin{array}{l}\text { J.A. Boyd } \\
\text { No. } 1\end{array}$ & $\begin{array}{l}\text { sec. } 34, \mathrm{~T} 27 \mathrm{~s}, \mathrm{R} 16 \mathrm{E} \\
\text { Tarpon springs, } 6 \\
\mathrm{mi} . \mathrm{SE}\end{array}$ & $30 \pm$ & & 1,161 & Drilling \\
\hline & $\begin{array}{l}\text { Coastal Petroleum } \\
\text { Company }\end{array}$ & $\begin{array}{l}\text { Ed C. Wright } \\
\text { No. } 1\end{array}$ & $\begin{array}{l}\text { Sec. } 7, \text { T30s, RI7E } \\
\text { St.Petersburg }, 9 \\
\text { mi. N }\end{array}$ & $2.4 \mathrm{Grd}$ & & & $\begin{array}{l}\text { Location, applica- } \\
\text { tion to drili re- } \\
\text { ceived Jan.29,1948. }\end{array}$ \\
\hline
\end{tabular}

\section{POLK COUNTY}

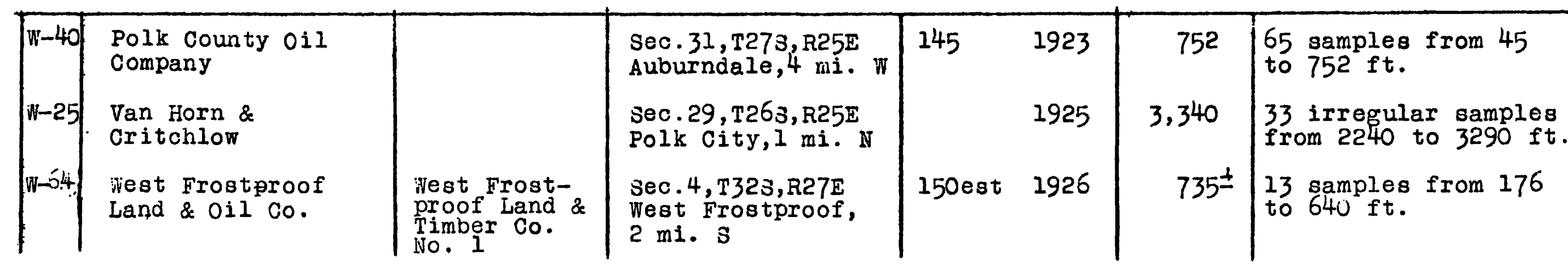


POLK COUHTY - Continued

\begin{tabular}{|c|c|c|c|c|c|c|c|}
\hline $\begin{array}{l}\text { FGS } \\
\text { NO. }\end{array}$ & CONPANY Or OWNERS & $\begin{array}{l}\text { FARM or } \\
\text { WELL NANEE }\end{array}$ & LOCATION & $\begin{array}{l}\text { ELEV- } \\
\text { ATION }\end{array}$ & $\begin{array}{l}\text { YEAR } \\
\text { COU- } \\
\text { PLETED } \\
\end{array}$ & DEFTH & RRMARKS \\
\hline$i-51$ & $\begin{array}{l}\text { Florida Pioneer } \\
\text { oil Corporation }\end{array}$ & $\begin{array}{l}\text { Heckscher- } \\
\text { Yarnell No.1 }\end{array}$ & $\begin{array}{l}\text { Sec. 28, T30 } 3, \text { R25E } \\
\text { Bar tow, } 5 \frac{1}{2} \mathrm{mi} \text {. SE }\end{array}$ & $108 \mathrm{Grd}$ & 1928 & 4,540 & $\begin{array}{l}431 \text { samples from } 20 \\
\text { to } 4540 \text { ft. }\end{array}$ \\
\hline
\end{tabular}

$\hat{n}$

PUTAYAL COUNTY

\begin{tabular}{|c|c|c|c|c|c|c|c|}
\hline$\frac{i-}{1514}$ & $\begin{array}{l}\text { Sun Oil Company- } \\
\text { Jeaboard Oil } \\
\text { Company }\end{array}$ & $\begin{array}{l}\text { Q.I. Roberts } \\
\text { No.1 \& } 1-A\end{array}$ & $\begin{array}{l}\text { sec. } 19, \mathrm{~T} 93, \mathrm{R} 25 \mathrm{E} \\
\text { Carraway, } 4 \mathrm{mi} \cdot \mathrm{it}\end{array}$ & $\begin{array}{l}196 \mathrm{Grd} \\
206 \mathrm{DF}\end{array}$ & 1947 & 3,327 & $\begin{array}{l}32 \text { samples from No.l } \\
\text { from o to } 320 \text { ft. } \\
204 \text { samples from No. } \\
1-A \text { from } 0 \text { to } 3327 \\
\text { ft. and one core No. } \\
1 \text { 3 } 3324-3326 \text {. Schlum } \\
\text { berger from } 281 \text { to } \\
3328 \text { Driller's log } \\
\text { from o to } 3328 \text {. }\end{array}$ \\
\hline
\end{tabular}


SANTA ROSA COUNTY

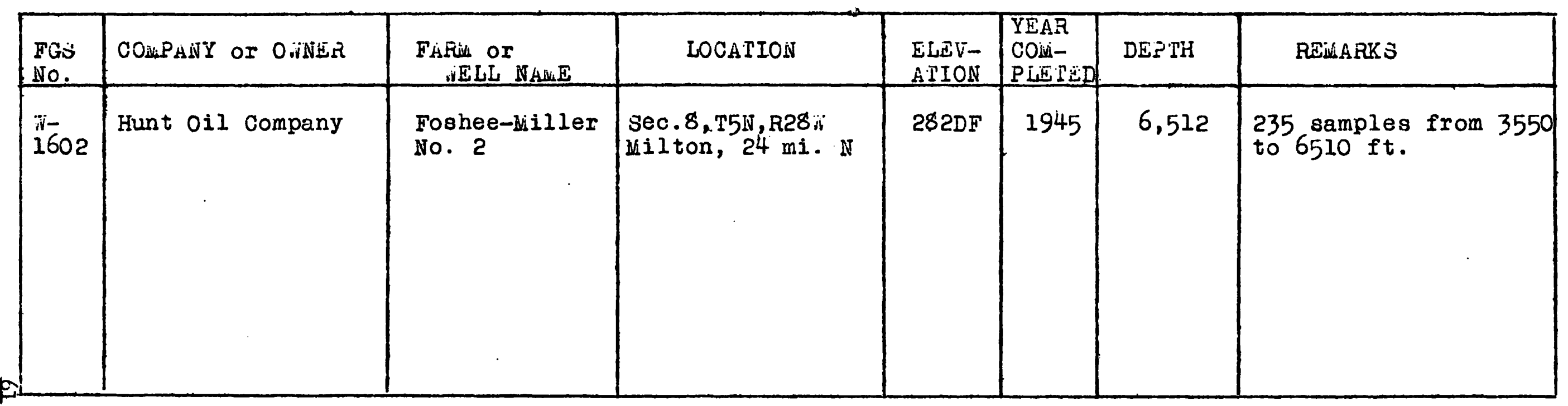

SARAZOTA COUNTY

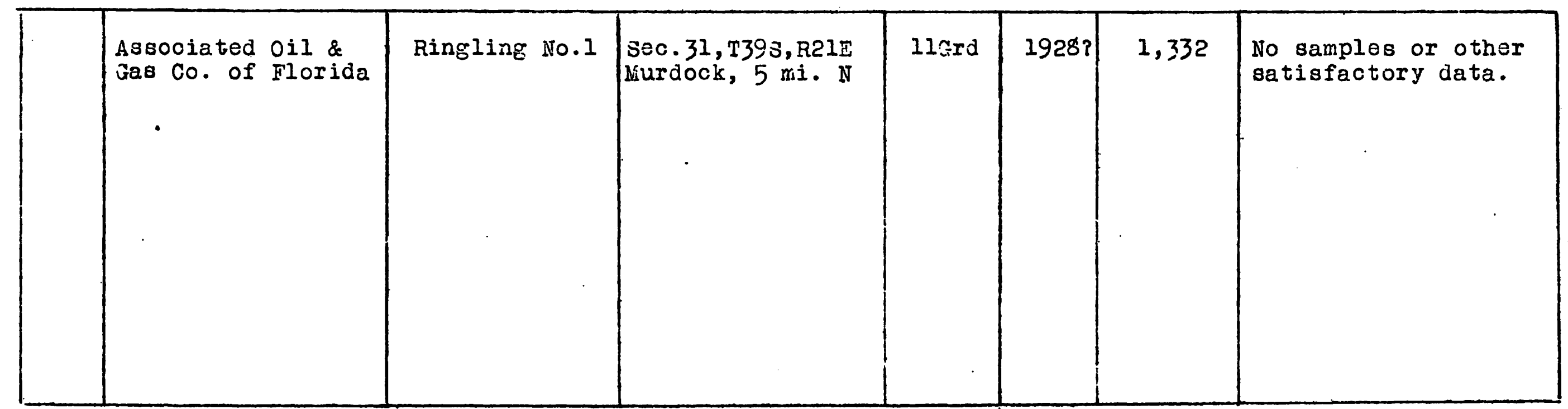


SUIAINNEE COUNTY

\begin{tabular}{|c|c|c|c|c|c|c|c|}
\hline $\begin{array}{l}\text { FGS } \\
\text { WO. }\end{array}$ & COMLPANY OI OWNERS & $\begin{array}{l}\text { FARR Or } \\
\text { WELL NAME }\end{array}$ & LOCATION & $\begin{array}{l}\text { ELEV- } \\
\text { ATION }\end{array}$ & $\begin{array}{l}\text { YEAR } \\
\text { COM- } \\
\text { PLETED } \\
\end{array}$ & DEPTH & REMARKS \\
\hline 358 & $\begin{array}{l}\text { Tm.G.Blanchard et } \\
\text { al }\end{array}$ & $\begin{array}{l}\text { Lucy Cotton } \\
\text { No. } 1\end{array}$ & $\begin{array}{l}\text { Sec. } 35, T 3 S, R I 1 E \\
\text { Luravilie, } 4 \mathrm{mi} \\
\text { Ni }\end{array}$ & $70 \pm$ & 1937 & $202 \frac{1}{2}$ & $\begin{array}{l}34 \text { samples from sur- } \\
\text { face to } 180 \mathrm{ft} \text {. }\end{array}$ \\
\hline 493 & $\begin{array}{l}\text { J. J.Phillips } 0 i l \\
\text { Explorations } \\
\text {. }\end{array}$ & $\begin{array}{l}\text { Shilkett } \\
\text { No. } 1\end{array}$ & $\begin{array}{l}\text { Sec.14, T4S, RI } 4 \mathrm{E} \\
\text { McAlpin, } 3 \frac{1}{2} \mathrm{mi} . \\
\mathrm{E}\end{array}$ & 99 & 1939 & 612 & $\begin{array}{l}\text { Il samples from } 20 \\
\text { to } 350 \mathrm{ft} \text {. }\end{array}$ \\
\hline 1450 & Sun oil company & $\begin{array}{l}\text { Earl Odom et } \\
\text { al No. } 1\end{array}$ & $\begin{array}{l}\text { Sec. } 31, \mathrm{~T} 5 \mathrm{~S}, \mathrm{R} 15 \mathrm{E} \\
\text { Branford, } 7 \mathrm{mi} \text {. } \\
\mathrm{NE}\end{array}$ & $\begin{array}{l}\text { 68Grd } \\
73 \mathrm{DF}\end{array}$ & 1947 & 3,157 & $\begin{array}{l}175 \text { samples from } 49 \\
\text { to } 3150 \text { ft. Gap in } \\
\text { sainples from } 75 \text { to } \\
1440 \text { ft. Cores from } \\
\text { No. } 13 \text { 2897-2902 to } \\
\text { core No. } 9 \text { 3 } 3152- \\
3157.5021 \text { umberger } \\
\text { from } 57 \text { to } 3161 \text {. } \\
\text { Driller's log from } \\
\text { o to } 3157 \text {. }\end{array}$ \\
\hline 11548 & sun oil Company & $\begin{array}{l}\text { J.H. Tillis } \\
\text { iNo. I }\end{array}$ & $\begin{array}{l}\text { Sec. } 28, \text { T2S, Rl5E } \\
\text { Houston, } 6 \mathrm{mi} \text {. } \\
\text { NE }\end{array}$ & $\begin{array}{l}152 \mathrm{Grd} \\
162 \mathrm{DF}\end{array}$ & 1947 & 3,568 & $\begin{array}{l}23 \text { samples from o } \\
\text { to } 225 \text { ft. No samp- } \\
\text { les from } 225 \text { to } 1450 \\
\text { ft. } 205 \text { samples from } \\
1450 \text { to } 3560 \text { ft. Cores } \\
\text { from No. } \$ 3092-3100 \\
\text { to ivo. } 44 \text { \$ } 3552-3568 \text {. } \\
\text { Schlumberger from } 122 \\
\text { to } 3572 \text {. Driller's } \\
\text { log from } 0 \text { to } 3568 \text {. }\end{array}$ \\
\hline
\end{tabular}


VOLUSIIA COUNTY

\begin{tabular}{|c|c|c|c|c|c|c|c|}
\hline $\begin{array}{l}\text { FGS } \\
\text { No. }\end{array}$ & COULPANY Or OWINER & $\begin{array}{l}\text { FARHi or } \\
\text { TELLL NAWE }\end{array}$ & LOCATION & $\begin{array}{l}\text { ELEV- } \\
\text { ATION }\end{array}$ & $\begin{array}{l}\text { YEAR } \\
\text { CONI- } \\
\text { PLEIET } \\
\end{array}$ & DEPTH & REMARKS \\
\hline $\begin{array}{l}7118 \\
1118\end{array}$ & Sun Oil Company & $\begin{array}{l}\text { Powell Land Co. } \\
\text { No. } 1\end{array}$ & $\begin{array}{l}\text { Sec } 11, T 17 S, R 31 E \\
\text { De Lund, } 7 \frac{1}{2} \\
\text { mi. E }\end{array}$ & $\begin{array}{l}42 \mathrm{Grd} \\
48 \mathrm{DF}\end{array}$ & 1945 & 5,958 & 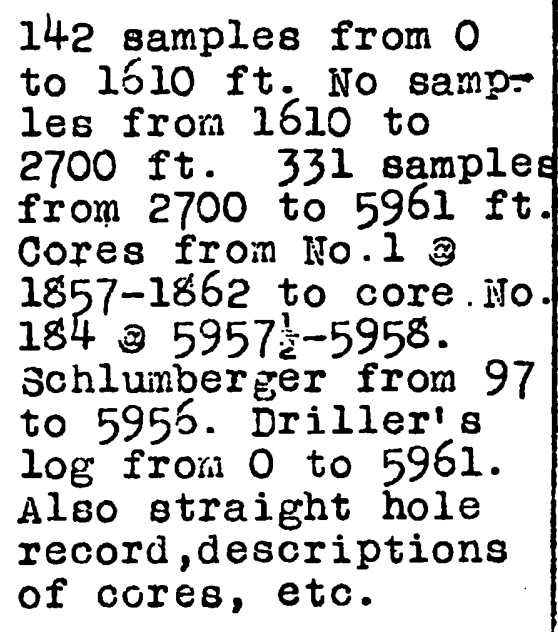 \\
\hline
\end{tabular}

WAKULLA COUNTY

\begin{tabular}{|c|c|c|c|c|c|c|c|}
\hline$\pi-12$ & $\begin{array}{l}\text { Bonheur Devel- } \\
\text { opment Company }\end{array}$ & Cates No. 1 & $\begin{array}{l}\text { Jec. } 16, \text { T3s, Rld } \\
\text { Burns, near } \\
\text { Wakulia }\end{array}$ & $17 \pm$ & 1919 & 2,169 & $\begin{array}{l}65 \text { samples from } 50 \text { to } \\
2153 \text { ft. Driller's log } \\
\text { from o to } 2159 \text {. Water }\end{array}$ \\
\hline 440 & Ravlin-Brown & $\begin{array}{l}\text { V.G.Phillips } \\
\text { No. } 1\end{array}$ & $\begin{array}{l}\text { Wec.14, T3S, RlE } \\
\text { Wakulla, } 2 \mathrm{mi} . \mathrm{E}\end{array}$ & 28 & 1943 & 5,765 & $\begin{array}{l}498 \text { samples from } 394 \\
\text { to } 5746 \mathrm{ft} \text {. }\end{array}$ \\
\hline
\end{tabular}




\begin{tabular}{|c|c|c|c|c|c|c|c|}
\hline $\begin{array}{l}\text { FGS } \\
\text { NO. }\end{array}$ & COMPANY Or OWIERS & $\begin{array}{l}\text { FARN Or } \\
\text { VELL NAME }\end{array}$ & LOCATION & $\begin{array}{l}\text { ELEV- } \\
\text { AIIONI }\end{array}$ & \begin{tabular}{|l|} 
YEAR \\
COW- \\
PLETED \\
\end{tabular} & DEPTH & REBARKS \\
\hline Wgi- & Florida oil co. & Rat Head well & $\begin{array}{l}\text { jec. } 16, \mathrm{~T} 3 \mathrm{i}, \mathrm{R} 20 \mathrm{~N}, \\
\text { DeFuniak Springs, } \\
7 \mathrm{mi} . \mathrm{W}\end{array}$ & $300 \pm$ & 1921 & 1,888 & $\begin{array}{l}\text { No sainples or other } \\
\text { satisfactory data. } \\
\text { Driller's log from } 0 \\
\text { to } 1388 .\end{array}$ \\
\hline$\sqrt{11-71}$ & Florida oil co. & Bruce well & $\begin{array}{l}\text { sec. } 22, T 1 \mathrm{H}, \mathrm{Rl} 7 \mathrm{n}, \\
\text { Bruce, } 2 \mathrm{mi} . \mathrm{E}\end{array}$ & 32 & 1921 & 770 & $\begin{array}{l}\text { Four samples from } \\
484 \text { to } 770 \text { ft. Drill- } \\
\text { er's log from o to } \\
770 \text {. }\end{array}$ \\
\hline$i-$ & Oil City Corp: & $\begin{array}{l}\text { Walton Land } \\
\& \text { Timber Co. } \\
\text { No. } 1\end{array}$ & $\begin{array}{l}\text { Sec.12, TlN, R19w, } \\
\text { DeFuniak Springs, } \\
10 \mathrm{mi} \text {. s }\end{array}$ & 200 & 1933 & 5,375 & $\begin{array}{l}179 \text { samples from } 13 \\
\text { to } 5337 \mathrm{ft} \text {. }\end{array}$ \\
\hline & $\begin{array}{l}\text { Sanford } \bar{c} \\
\text { Arrington }\end{array}$ & $\begin{array}{l}\text { Walton Land } \\
\& \text { Timber Co. } \\
\text { No. } 1\end{array}$ & $\begin{array}{l}\text { Sec.16, THN, Rl8m, } \\
\text { De Funiak Springs, } \\
12 \mathrm{mi} \text {. SE }\end{array}$ & $\begin{array}{l}185 \mathrm{Grd} \\
193 \mathrm{DF}\end{array}$ & 1942 & $5,85 ?$ & $\begin{array}{l}\text { No samples. Schlum- } \\
\text { berger from } 375 \text { to } \\
5330 \mathrm{ft} \text {. }\end{array}$ \\
\hline & $\begin{array}{l}\text { Sanford \& } \\
\text { Arrington }\end{array}$ & $\begin{array}{l}\text { Core test No. } \\
1-\text { i } \\
550^{\prime} \text { \& } 52^{\circ} \\
\text { of well No.1 }\end{array}$ & $\begin{array}{l}\text { Sec.16́, TlN, Rl8\%, } \\
\text { DeFuniak Springs, } \\
12 \mathrm{mi} \text {. SE }\end{array}$ & $195 \frac{ \pm}{\text { Grd }}$ & 1943 & 1,437 & $\begin{array}{l}\text { No samples or other } \\
\text { data. }\end{array}$ \\
\hline $\mathrm{W}_{739}$ & J.R. Sealy & $\begin{array}{l}\text { Victory Field } \\
\text { No. I }\end{array}$ & $\begin{array}{l}\mathrm{Sec} .5, \mathrm{~T} 2 \mathrm{~s}, \mathrm{Rl} 8 \mathrm{ij} \\
\text { Freeport, } 5 \mathrm{mi} . \mathrm{SE}\end{array}$ & $\begin{array}{l}17-18 \\
\text { Grd }\end{array}$ & 1944 & 757 & $\begin{array}{l}142 \text { samples from } 16 \\
\text { to } 753 \mathrm{ft} \text {. }\end{array}$ \\
\hline 842 & J.R.sealy & $\begin{array}{l}\text { Victory Field } \\
\text { No. } 2\end{array}$ & $\begin{array}{l}\text { sec.36, T1S, RI9 } \\
\text { Freeport, } 4 \mathrm{mi} . \mathrm{SE}\end{array}$ & & 1945 & 856 & $\begin{array}{l}118 \text { samples from sur- } \\
\text { face to } 810 \mathrm{ft} .\end{array}$ \\
\hline$\sqrt{499}$ & H.H.Givan et al & $\begin{array}{l}\text { Marion Corp. } \\
\text { No. } 1\end{array}$ & $\begin{array}{l}\text { Sec.Il, TlS, R2OH, } \\
\text { Portland, near }\end{array}$ & $50 \pm$ & 1943 & 2,757 & $\begin{array}{l}64 \text { samples from } 100 \\
\text { to } 2712 \text { ft. }\end{array}$ \\
\hline
\end{tabular}




\begin{tabular}{|c|c|c|c|c|c|c|c|}
\hline $\begin{array}{l}\text { FGS } \\
\text { HO. }\end{array}$ & COWPANY Or ONINER & $\begin{array}{l}\text { FARDi or } \\
\text { TELL NAWE }\end{array}$ & LONATION & $\begin{array}{l}\text { ELEV- } \\
\text { ATION }\end{array}$ & $\begin{array}{l}\text { YEAR } \\
\text { COU- } \\
\text { PLETED } \\
\end{array}$ & DEPTH & RIMARKS \\
\hline & J.R. Sealy & $\begin{array}{l}\text { Victory Field } \\
\text { No. } 3\end{array}$ & $\begin{array}{l}\sec .15, \mathrm{Tl}, \mathrm{R} 18 \mathrm{~W} \\
\text { Freeport, } 7 \mathrm{mi} \text {. E }\end{array}$ & & 1946 & 200 & $\begin{array}{l}\text { No samples or otiner } \\
\text { data. }\end{array}$ \\
\hline 1086 & $\begin{array}{l}\text { R.D. Logan, Trustee } \\
\text { for S. . Breeding } \\
\text { and Thomas O.Payne }\end{array}$ & $\begin{array}{l}\text { Nalton Land \& } \\
\text { Timber Co. } \\
\text { No. } 1\end{array}$ & 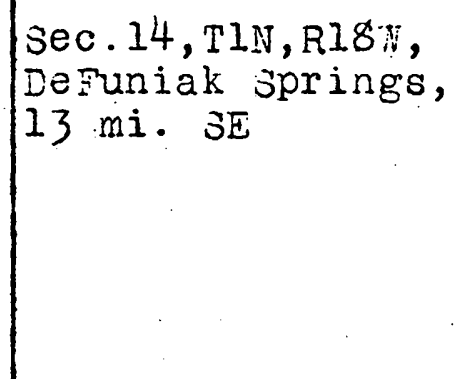 & 200 est. & 1946 & 6,505 & $\begin{array}{l}244 \text { samples from } \$ 48 \\
\text { to } 6505 \text { ft. Four } \\
\text { cores from No. } 4 \text { 3 } \\
4056-4076 \text { to core No. } \\
7 \text { 3 } 4812-4832 . \text { schlum } \\
\text { berser from } \$ 46 \text { to } \\
6503 \text {. }\end{array}$ \\
\hline 1535 & Hunt oil Company & $\begin{array}{l}\text { Geeher Lin- } \\
\text { ton ivo. } 1\end{array}$ & 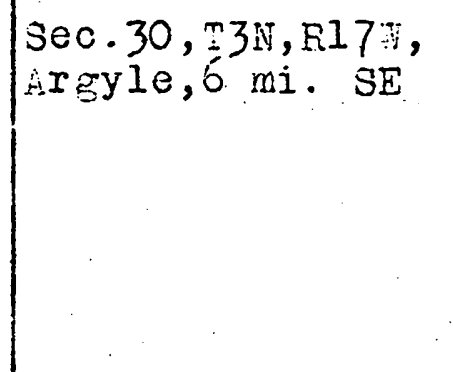 & $\begin{array}{r}97 \mathrm{Grd} \\
104 \mathrm{DI}\end{array}$ & 1947 & 6.503 & $\begin{array}{l}429 \text { samples from } 15 \\
\text { to } 6500 \text { rt. Cores } \\
\text { from No. I } 3930- \\
3840 \text { to core } 110.24 \text {. } \\
5960-5957 \text {. Schlumber- } \\
\text { ger from } 294 \text { to } 6503 . \\
\text { Driller' s log fron } 0 \\
\text { to } 6505 .\end{array}$ \\
\hline 1591 & D. . I. Byers & $\begin{array}{l}\text { Earl Edwards } \\
\text { No. } 1\end{array}$ & $\begin{array}{l}\text { sec. } 28, \mathrm{~T} 5 \mathrm{~N}, \mathrm{R} 2 \mathrm{lin}, \\
\text { Lakewood, } 7 \mathrm{mi} \text {. SW }\end{array}$ & $2086 r d$ & 1947 & 5,270 & $\begin{array}{l}404 \text { samples from } 20 \\
\text { to } 5250 \text { ft. Cores } \\
\text { from ino. I } 3992- \\
4012 \text { to core No. } 4 \text { 3 } \\
4789-4805 \text {. Schlumber- } \\
\text { ger from } 105 \text { to } 5267 . \\
\text { Driller's } 10 \mathrm{~g} \text { from } \\
0 \text { to } 5270 \text {. }\end{array}$ \\
\hline
\end{tabular}


WALTON COUHTY - Continued

\begin{tabular}{|c|c|c|c|c|c|c|c|}
\hline $\begin{array}{l}\text { FGSO } \\
\text { iNo. }\end{array}$ & CONPSHY OY OHNERO & $\begin{array}{c}\text { FAFi: or } \\
\text { BELL NANE }\end{array}$ & LOCATION & $\begin{array}{l}\text { ELEV- } \\
\text { ATION }\end{array}$ & \begin{tabular}{|l|} 
YEAR \\
COND- \\
PLETED \\
\end{tabular} & DEPTH & REMARKS̈ \\
\hline 1657 & $\begin{array}{l}\text { D.E.i. Byers } \\
\text { jatterlee \& Adams } \\
\text { Driling Co. }\end{array}$ & $\begin{array}{l}\text { J.R. Soaley } \\
\text { No. I } \\
\text { Nalton Land \& } \\
\text { Timber Co.NO.I }\end{array}$ & 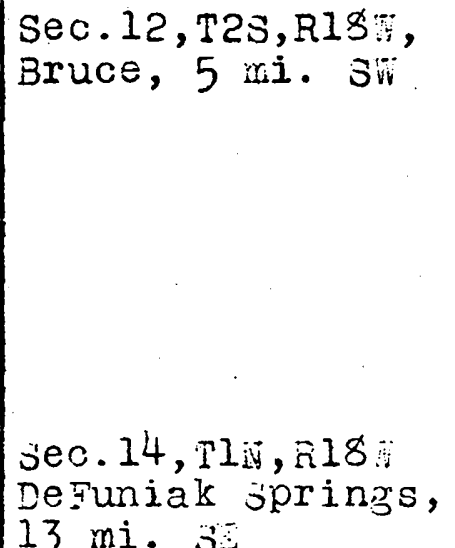 & $\begin{array}{l}27 \text { ard } \\
37 \mathrm{DF}\end{array}$ & 1948 & 5,475 & $\begin{array}{l}\text { Spudded Dec. } 15,1947 . \\
\text { Completed Jan. } 13 \text {, } \\
\text { l948. } 555 \text { samples } \\
\text { from } 20 \text { to } 5480 \text { ft. } \\
\text { Cores from } 10.13 \\
4194-4199 \text { to core } \\
\text { No.3 } 3985-4995 \text {. } \\
\text { 3chiumberger from } \\
133 \text { to } 5477 . \text { Drillers } \\
\text { log from o to } 5475 . \\
\text { Location. Application } \\
\text { approved Feb. } 4,1948\end{array}$ \\
\hline
\end{tabular}

WAOHTHGTON COUNTY

\begin{tabular}{|c|c|c|c|c|c|c|c|}
\hline$i^{i-1}$ & $\begin{array}{l}\text { Chipley Oil Conpany } \\
\text { Stump, Dekle and } \\
\text { Thomas } \\
\text { Stump, Dekle and } \\
\text { Thomas }\end{array}$ & Dekle No.l & 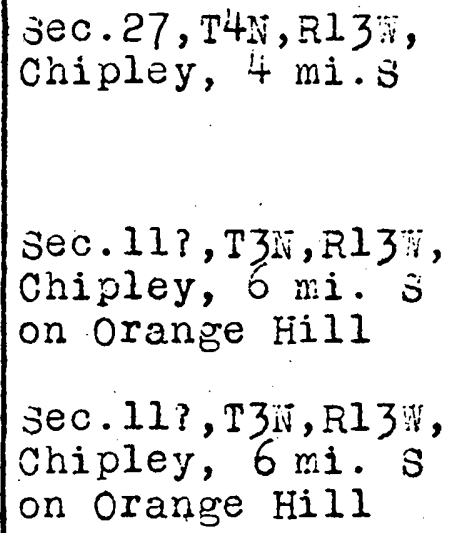 & 200 & $\begin{array}{l}1907 \text { or } \\
1908 \\
19070 n \\
1908\end{array}$ & $\begin{array}{l}1,600 \pm \\
1,800 \pm\end{array}$ & $\begin{array}{l}1796 \text { cuttings( } 2 \text { sets) } \\
\text { from } 5 \text { to } 4910 \text { ft. } \\
\text { An excellently samp- } \\
\text { led well. } \\
\text { No samples or drill- } \\
\text { er's log. Lost bit. } \\
\text { Abandoned. } \\
\text { ivo samples or drill- } \\
\text { er's log. }\end{array}$ \\
\hline
\end{tabular}



